\title{
Total Synthesis of the Bacterial Diisonitrile Chalkophore SF2768
}

\author{
Yao $\mathrm{Xu}^{\dagger}$, Derek S. Tan ${ }^{\dagger,+*}$ \\ ${ }^{\dagger}$ Chemical Biology Program, Sloan Kettering Institute and ${ }^{\ddagger}$ Tri-Institutional Research Program, \\ Memorial Sloan Kettering Cancer Center, New York, New York 10065, United States
}

\section{Supporting Information}
A. Supplementary Figures
$\mathrm{S} 2$
B. Materials and Methods
$\mathrm{S} 3$
C. Synthesis of NHS Ester 5
S5
D. Synthesis of Azidoalcohols 8
S7
E. Synthesis of SF2768 (2L,5S-1) and 5-epi-SF2768 (2L,5R-1)
$\mathrm{S} 10$
F. Comparison of NMR Data for Synthetic SF2768, 5-epi-SF2768, and Literature Reports
G. Synthesis of Acyclic Diisonitriles 3 and 4 and Comparison of NMR Data $\quad$ S23
H. Formation and Characterization of $\mathrm{Cu}(\mathrm{I})$ Complexes of Diisonitriles
$\mathrm{S} 29$
I. ${ }^{1} \mathrm{H}-\mathrm{NMR}$ Titration of Diisonitrile Acetate $\mathbf{4}$ with $\mathrm{Cu}(\mathrm{I})$
S37
J. UV-Vis Competitive Titration Experiments of $\mathrm{Cu}(\mathrm{I})$ Complexes with BCS S39
K. Attempted Formation of Zn(II) Complex of Diisonitrile Acetate $4 \quad$ S45
L. ${ }^{1} \mathrm{H}-\mathrm{NMR}$ and ${ }^{13} \mathrm{C}-\mathrm{NMR}$ Spectra 


\section{A. SUPPLEMENTARY FigURES}
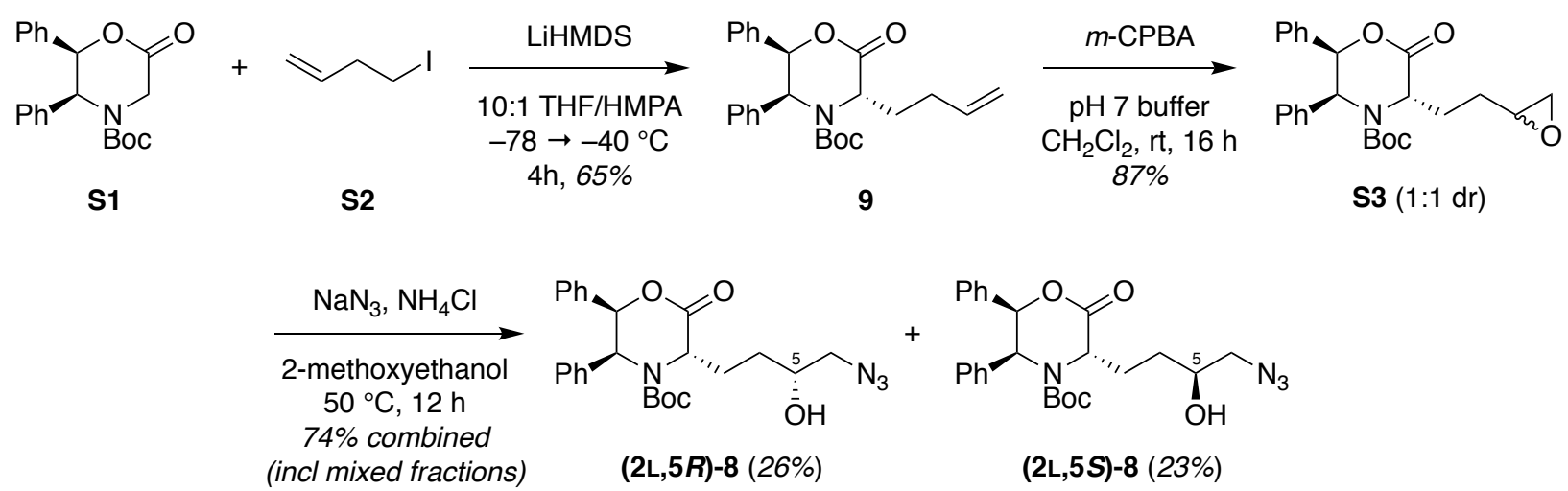

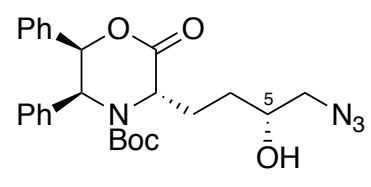

(2L,5R)-8 (26\%)<smiles>N[C@H](O)CC[C@H]1C(=O)O[C@@H](c2ccccc2)[C@H](c2ccccc2)N1[PH3]</smiles>

(2L,5S)-8 (23\%)

Figure S1. Synthesis of azidoalcohols 8.

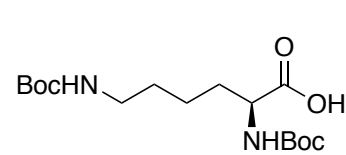

S4

1) $i-\mathrm{BuOCOCl}$

$i-\mathrm{Pr}_{2} \mathrm{NEt}, \mathrm{THF}$

$-10^{\circ} \mathrm{C}, 40 \mathrm{~min}$

2) $\mathrm{NaBH}_{4}$, then $\mathrm{H}_{2} \mathrm{O}$

$-10^{\circ} \mathrm{C} \rightarrow \mathrm{rt}, 1 \mathrm{~h}$

$75 \%$

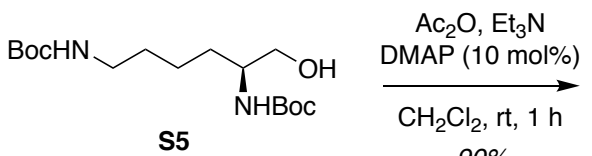

$90 \%$

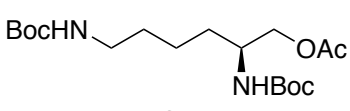

S6

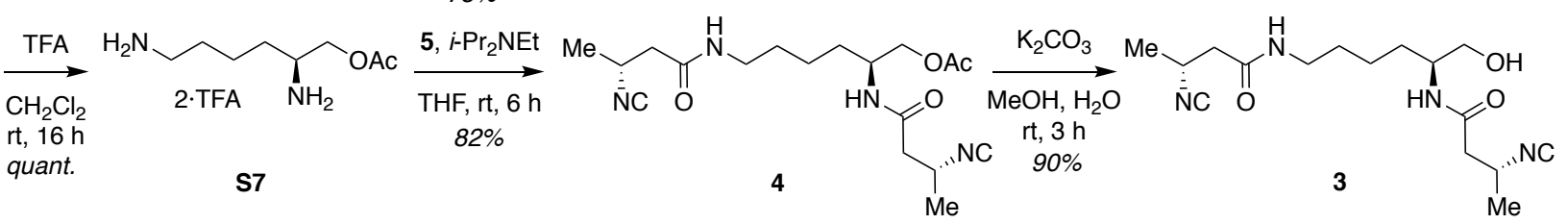

Figure S2. Synthesis of linear diisonitriles 3 and 4. 


\section{B. Materials ANd Methods}

\section{Reagents:}

Reagents were obtained from Sigma-Aldrich (www.sigma-aldrich.com) or Acros Organics (www.fishersci.com) and used without further purification except as listed below.

4-Bromobut-1-ene was purchased from Oakwood and used as received.

$N^{2}, N^{6}$-bis(tert-butoxycarbonyl)-L-lysine was purchased from Alfa Aesar and used as received.

(R)-3-aminobutanoic acid was purchased from Combi-Blocks and used as received.

Sodium borohydride was purchased from Combi-Blocks and used as received.

tert-Butyl (2R,3S)-6-oxo-2,3-diphenylmorpholine-4-carboxylate (S1) was purchased from AK Scientific and used as received.

Optima or HPLC grade solvents were obtained from Fisher Scientific (www.fishersci.com), degassed with Ar, and purified on a solvent drying system as described ${ }^{1}$ unless otherwise indicated. Air sensitive reactions were performed in flame-dried glassware under positive Ar pressure with magnetic stirring. Liquid reagents and solutions were transferred through rubber septa via syringes flushed with Ar prior to use.

\section{Reactions}

All reactions were performed in flame-dried glassware under positive Ar pressure with magnetic stirring unless otherwise noted. Liquid reagents and solutions were transferred thru rubber septa via syringes flushed with Ar prior to use. Cold baths were generated as follows: $0{ }^{\circ} \mathrm{C}$, wet ice/water; $-10{ }^{\circ} \mathrm{C}$, wet ice/brine; $-20^{\circ} \mathrm{C}$, dry ice/isopropanol monitored with a thermometer; $44{ }^{\circ} \mathrm{C}$, dry ice $/ \mathrm{CH}_{3} \mathrm{CN}$; $-63{ }^{\circ} \mathrm{C}$, dry ice/chloroform; $-78{ }^{\circ} \mathrm{C}$, dry ice/acetone; $-100{ }^{\circ} \mathrm{C}$, dry ice/ $\mathrm{Et}_{2} \mathrm{O}$.

\section{Chromatography}

TLC was performed on $0.25 \mathrm{~mm}$ E. Merck silica gel $60 \mathrm{~F} 254$ plates and visualized under UV light $(254 \mathrm{~nm})$ or by staining with potassium permanganate $\left(\mathrm{KMnO}_{4}\right)$. Silica flash chromatography was performed on E. Merck 230-400 mesh silica gel 60. Analytical LC-MS was carried out on a Waters Acuity SQD LC-MS in electrospray ionization (ESI) mode, with a XBridge C18 reverse phase column $(4.6 \times 150 \mathrm{~mm}, 4 \mu \mathrm{m})$ using a flow rate of $1.2 \mathrm{~mL} / \mathrm{min}$ and a gradient of $5-95 \% \mathrm{CH}_{3} \mathrm{CN}$ in $0.1 \%$ aq TFA or none TFA condition over $12 \mathrm{~min}$. Lyophilization of larger aqueous samples was performed using a Labconco Freezone 2.5 instrument.

\section{Analytical Instrumentation}

Melting point determinations were performed on an Electrothermal 9100 apparatus and are uncorrected. Optical rotations were recorded on a JASCO model P-1020 digital polarimeter with P-103T temperature controller. IR spectra were recorded on a Bruker Optics Tensor 27 FTIR spectrometer using an attenuated total reflection (ATR) attachment with peaks reported in $\mathrm{cm}^{-1}$. UV-Vis spectra were recorded on a SpectraMax M5 microplate reader from Molecular Devices

\footnotetext{
${ }^{1}$ Pangborn, A. B.; Giardello, M. A.; Grubbs, R. H.; Rosen, R. K.; Timmers, F. J. Organometallics 1996, 15, 15181520.
} 
LLC. Sample were prepared in a Costar 96-well black, clear-bottom multiplate. Samples were measured relative to their reference solvent. Data was analyzed using GraphPad Prism 6. NMR spectra were recorded on a Bruker UltraShield Plus $500 \mathrm{MHz}$ Avance III NMR or UltraShield Plus $600 \mathrm{MHz}$ Avance III NMR with $\mathrm{DCH}$ CryoProbe at $24{ }^{\circ} \mathrm{C}$ in $\mathrm{CDCl}_{3}$ unless otherwise indicated. Chemical shifts are expressed in ppm relative to TMS $\left({ }^{1} \mathrm{H}, 0 \mathrm{ppm}\right)$ or solvent signals: $\mathrm{CDCl}_{3}\left(\mathrm{CDCl}_{3}: \delta 7.26 \mathrm{ppm},{ }^{13} \mathrm{C}, 77.16 \mathrm{ppm}\right), d_{6}$-DMSO $\left(\delta 2.50 \mathrm{ppm},{ }^{13} \mathrm{C}, 39.52 \mathrm{ppm}\right), \mathrm{CD}_{3} \mathrm{OD}$ $\left(\delta 3.31 \mathrm{ppm},{ }^{13} \mathrm{C}, 49.00 \mathrm{ppm}\right)$. Data are reported as follows: chemical shift, multiplicity $(\mathrm{s}=$ singlet, $\mathrm{d}=$ doublet, $\mathrm{t}=$ triplet, $\mathrm{q}=$ quartet, $\mathrm{p}=$ pentet, $\mathrm{br}=$ broad, $\mathrm{m}=$ multiplet), coupling constants $(\mathrm{Hz})$ and integration. NMR spectra were processed using Bruker TopSpin, Mnova (www.mestrelab.com/software/mnova-nmr) software. Mass spectra were obtained at the MSKCC Analytical Core Facility on a Waters Acuity SQD LC-MS or PE SCIEX API 100 by electrospray (ESI) ionization or atmospheric pressure chemical ionization (AP-CI). High resolution mass spectra were obtained on a Waters Acuity Premiere XE TOF LC-MS by electrospray ionization (ESI). 


\section{SYNTHESIS OF NHS ESTER 5}

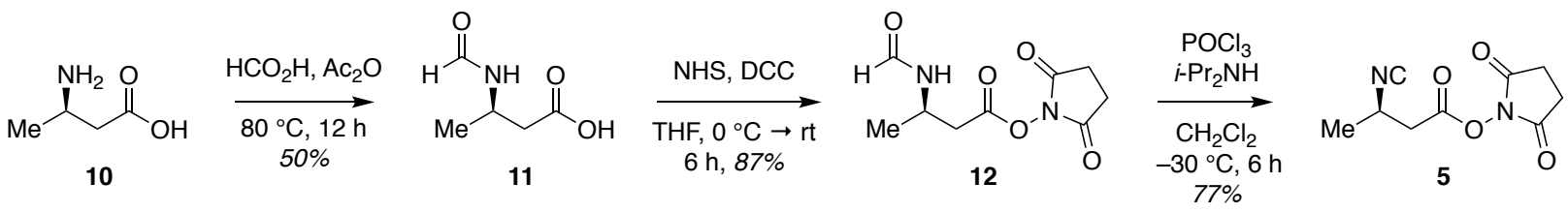

Figure S3. Synthesis of NHS ester 5.<smiles>C[C@H](CC(=O)O)NC(=O)O</smiles>

(R)-3-Formamidobutanoic acid (11). Synthesized by a modified version of the general protocol reported in the literature. ${ }^{2}$ In a $100-\mathrm{mL}$ roundbottom flask, $(R)$-3-aminobutanoic acid (10) $(2.50 \mathrm{~g}, 24.2 \mathrm{mmol}, 1.0$ equiv) was dissolved in formic acid (15 mL), acetic anhydride (10 $\mathrm{mL})(\mathrm{v} / \mathrm{v}=1.5: 1)$ and heated to $80{ }^{\circ} \mathrm{C}$ for $12 \mathrm{~h}$. The mixture was cooled to $\mathrm{rt}$ and concentrated by rotary evaporation. The crude product was triturated in $\approx 10: 1$ hexanes/EtOAc to afford pure formamide 11 as a white solid (1.58 g, $50 \%$ yield).

mp: $81.0-82.0^{\circ} \mathrm{C}$. Optical Rotation: $[\alpha]_{D}^{17}:+10.0^{\circ}(c 0.55, \mathrm{MeOH})$. IR (ZnSe, film): 3305 (m), 2980 (w), 1715 (m), 1647 (s), 1538 (m), $1383(\mathrm{~m}), 1197(\mathrm{~m}) \mathrm{cm}^{-1}$. ${ }^{\mathbf{1}} \mathbf{H}-\mathbf{N M R}(600 \mathrm{MHz}$, $\left.\mathrm{CD}_{3} \mathrm{OD}\right): \delta 8.05$ (s) (rotamer), $\delta 7.97(\mathrm{~s}, 1 \mathrm{H}), 4.32(\mathrm{q}, J=6.7 \mathrm{~Hz}, 1 \mathrm{H}), 2.54(\mathrm{dd}, J=15.7,6.5 \mathrm{~Hz}$, $1 \mathrm{H}), 2.43(\mathrm{dd}, J=15.7,6.8 \mathrm{~Hz}, 1 \mathrm{H}), 1.25(\mathrm{~d}, J=6.7 \mathrm{~Hz})$ (rotamer), $1.22(\mathrm{~d}, J=6.7 \mathrm{~Hz}, 3 \mathrm{H})$. ${ }^{13}$ C-NMR (150 MHz, CD $\left.{ }_{3} \mathrm{OD}\right): \delta$ 174.5, 166.6 (rotamer), 162.9, 46.9 (rotamer), 42.5, 41.3, 21.9 (rotamer), 20.4. ESI-MS: Calcd for $\mathrm{C}_{5} \mathrm{H}_{9} \mathrm{NO}_{3} \mathrm{Na}\left([\mathrm{M}+\mathrm{Na}]^{+}\right)$: 154.0480, Found: 154.0477.<smiles>C[C@H](CC(=O)ON1C(=O)CCC1=O)NC=O</smiles>

2,5-Dioxopyrrolidin-1-yl (R)-3-formamidobutanoate (12). In a 50-mL roundbottom flask, (R)-3-formamidobutanoic acid (11) $(1.20 \mathrm{~g}, 9.15 \mathrm{mmol}, 1.0 \mathrm{equiv})$ was dissolved in THF $(13 \mathrm{~mL}, 0.7 \mathrm{M})$ and cooled to $0{ }^{\circ} \mathrm{C}$. 1-Hydroxypyrrolidine-2,5-dione (1.05 g, $9.15 \mathrm{mmol}, 1.0$ equiv) and $N, N^{\prime}$-dicyclohexylcarbodiimide (1.89 g, $9.15 \mathrm{mmol}, 1.0$ equiv) were added. The resulting mixture was stirred at $0{ }^{\circ} \mathrm{C}$ for $30 \mathrm{~min}$ then warmed to $\mathrm{rt}$ and stirred for $6 \mathrm{~h}$. The resulting white solids were removed by filtration and the filtrate was concentrated by rotary evaporation. $\mathrm{CH}_{3} \mathrm{CN}(20 \mathrm{~mL})$ was added to precipitate the dicyclohexylurea byproduct. The mixture was filtered and concentrated by rotary evaporation. Purification by silica flash chromatography (100\% EtOAc) yielded the NHS ester 12 as a light-yellow greasy oil (1.82 g, $87 \%$ yield).

TLC: $\mathrm{R}_{\mathrm{f}} 0.35\left(100 \%\right.$ EtOAc, $\left.\mathrm{KMnO}_{4}\right)$. Optical Rotation: $[\alpha]_{D}^{17}:+85.5^{\circ}\left(c 0.50, \mathrm{CHCl}_{3}\right)$. IR (ZnSe, film): 1814 (w), 1784 (w), 1732 (s), 1664 (m), 1524 (w), 1386 (w), 1203 (m), 1064 (m)

\footnotetext{
${ }^{2}$ Mizuno, Y.; Uehara, T.; Hanaoka, H.; Endo, Y.; Jen, C.-W.; Arano, Y. J. Med. Chem. 2016, 59 (7), 3331.
} 
$\mathrm{cm}^{-1} \cdot{ }^{1} \mathbf{H}-\mathbf{N M R}\left(600 \mathrm{MHz}, \mathrm{CDCl}_{3}\right): \delta 8.12(\mathrm{~d}, J=1.6 \mathrm{~Hz}, 1 \mathrm{H}), 6.13(\mathrm{~d}, J=8.5 \mathrm{~Hz}, 1 \mathrm{H}), 4.63-$ $4.46(\mathrm{~m}, 1 \mathrm{H}), 2.93(\mathrm{dd}, J=15.0,5.7 \mathrm{~Hz}, 1 \mathrm{H}), 2.89-2.83(\mathrm{~m}, 4 \mathrm{H}), 2.80(\mathrm{dd}, J=15.1,4.7 \mathrm{~Hz}$, $1 \mathrm{H}), 1.35(\mathrm{~d}, J=6.9 \mathrm{~Hz}, 3 \mathrm{H}) .{ }^{13} \mathbf{C}-\mathbf{N M R}\left(150 \mathrm{MHz}, \mathrm{CDCl}_{3}\right): \delta 166.3,160.7,40.7,37.2,25.7$, 25.7, 19.5. (Note: Minor peaks in the ${ }^{1} \mathrm{H}$ and ${ }^{13} \mathrm{C}$ NMR spectra are due to rotamers, which are not listed above.) ESI-MS: Calcd for $\mathrm{C}_{9} \mathrm{H}_{12} \mathrm{~N}_{2} \mathrm{O}_{5} \mathrm{Na}\left([\mathrm{M}+\mathrm{Na}]^{+}\right)$: 251.0644, Found: 251.0638 .<smiles>CC(C)CC(=O)ON1C(=O)CCC1=O</smiles>

2,5-Dioxopyrrolidin-1-yl (R)-3-isocyanobutanoate (5). Synthesized according to the general protocol reported in the literature. ${ }^{3}$ In a $100-\mathrm{mL}$ roundbottom flask, 2,5-dioxopyrrolidin-1-yl (R)-3-formamidobutanoate (12) (6.38 g, $27.95 \mathrm{mmol}, 1.0$ equiv) was dissolved in $\mathrm{CH}_{2} \mathrm{Cl}_{2}$ (40 $\mathrm{mL}, 0.7 \mathrm{M})$ and diisopropylamine $(7.64 \mathrm{~g}, 75.46 \mathrm{mmol}, 2.7 \mathrm{equiv})$. The mixture was cooled to $30{ }^{\circ} \mathrm{C}$ and $\mathrm{POCl}_{3}(5.14 \mathrm{~g}, 33.54 \mathrm{mmol}, 1.2$ equiv) was added dropwise. The mixture was stirred at $-30{ }^{\circ} \mathrm{C}$ for approximately $6 \mathrm{~h}$. Satd aq $\mathrm{NaHCO}_{3}(40 \mathrm{~mL})$ was added at $-30{ }^{\circ} \mathrm{C}$ and the resulting mixture was warmed to $\mathrm{rt}$ and extracted with $\mathrm{CH}_{2} \mathrm{Cl}_{2}(3 \mathrm{x} 40 \mathrm{~mL})$. The combined organic extracts were washed with brine $(200 \mathrm{~mL})$, dried $\left(\mathrm{Na}_{2} \mathrm{SO}_{4}\right)$ and concentrated by rotary evaporation. Purification by silica flash chromatography (50\% EtOAc in hexanes) yielded the isonitrile 5 as a white solid (4.52 g, 77\% yield).

TLC: $\mathrm{R}_{\mathrm{f}} 0.35\left(50 \%\right.$ EtOAc in hexanes, $\left.\mathrm{KMnO}_{4}\right)$. mp: $122-123{ }^{\circ} \mathrm{C}$. Optical Rotation: $[\alpha]_{D}^{17}$ : $31.3^{\circ}$ (c 0.50, $\mathrm{CHCl}_{3}$ ). IR (ZnSe, film): 2147 (w), 1818 (w), 1739 (s), $1372(\mathrm{w}), 1203(\mathrm{~m}), 1068$ (m), $754(\mathrm{~s}) \mathrm{cm}^{-1} .{ }^{1} \mathbf{H}-\mathbf{N M R}\left(600 \mathrm{MHz}, \mathrm{CDCl}_{3}\right): \delta 4.16(\mathrm{~h}, J=6.5 \mathrm{~Hz}, 1 \mathrm{H}), 3.10(\mathrm{dd}, J=16.4$, $6.5 \mathrm{~Hz}, 1 \mathrm{H}), 2.90(\mathrm{dd}, J=16.6,7.3 \mathrm{~Hz}, 2 \mathrm{H}), 2.87-2.82(\mathrm{~m}, 4 \mathrm{H}), 1.55(\mathrm{~d}, J=6.5 \mathrm{~Hz}, 3 \mathrm{H})$. ${ }^{13}$ C-NMR (150 MHz, $\left.\mathrm{CDCl}_{3}\right): \delta 168.7,164.7,157.7(\mathrm{t}, J=3.9 \mathrm{~Hz}), 46.0(\mathrm{t}, J=6.6 \mathrm{~Hz}), 38.4$, 25.7, 21.4. ESI-MS: Calcd for $\mathrm{C}_{9} \mathrm{H}_{10} \mathrm{~N}_{2} \mathrm{O}_{4} \mathrm{Na}\left([\mathrm{M}+\mathrm{Na}]^{+}\right)$: 233.0538, Found: 233.0529 .

\footnotetext{
${ }^{3}$ Buyck, T.; Wang, Q.; Zhu, J. J. Am. Chem. Soc. 2014, 136 (32), 11524.
} 


\section{SyNTHESIS OF AZIDOALCOHOLS 8}

$$
\sim^{\prime}
$$

4-Iodobut-1-ene (S2): Prepared as previously reported. ${ }^{4}$

${ }^{1}$ H-NMR $\left(500 \mathrm{MHz}, \mathrm{CDCl}_{3}\right): \delta 5.87-5.59(\mathrm{~m}, 1 \mathrm{H}), 5.26-4.86(\mathrm{~m}, 2 \mathrm{H}), 3.18(\mathrm{t}, J=7.2 \mathrm{~Hz}$, $2 \mathrm{H}), 2.62(\mathrm{qt}, J=7.0,1.4 \mathrm{~Hz}, 2 \mathrm{H}) .{ }^{13} \mathbf{C}$ NMR $\left(125 \mathrm{MHz}, \mathrm{CDCl}_{3}\right): \delta 137.0,117.1,37.8,4.8$.<smiles>C=CCC[C@H]1C(=O)OC(c2ccccc2)[C@H](c2ccccc2)[C@H]1c1ccccc1</smiles>

tert-Butyl (3S,5S,6R)-3-(but-3-en-1-yl)-2-ox0-5,6-diphenylmorpholine-4-carboxylate (9). Synthesized by a modified version of the literature protocol. ${ }^{5}$ In a $200-\mathrm{mL}$ roundbottom flask, tert-butyl (2R,3S)-6-oxo-2,3-diphenylmorpholine-4-carboxylate (S1, AK Scientific) (3.59 g, $10.16 \mathrm{mmol}, 1$ equiv) was dissolved in THF $(40 \mathrm{~mL})$ and HMPA $(4 \mathrm{~mL})(10: 1 \mathrm{v} / \mathrm{v})$. The mixture was cooled to $-78^{\circ} \mathrm{C}$ and LiHMDS (1 M in THF, $15.24 \mathrm{~mL}, 15.24 \mathrm{mmol}, 1.5$ equiv) was added dropwise. The mixture was stirred at $-78{ }^{\circ} \mathrm{C}$ for $45 \mathrm{~min}$, then 4-iodobut-1-ene (S2) $(9.25 \mathrm{~g}$, $50.82 \mathrm{mmol}, 5$ equiv) was added. The mixture was warmed to $-40{ }^{\circ} \mathrm{C}$ and stirred for an additional $4 \mathrm{~h}$. The reaction was quenched by addition of satd aq $\mathrm{NH}_{4} \mathrm{Cl}(70 \mathrm{~mL})$ and the aq phase was extracted with EtOAc $(3 \times 60 \mathrm{~mL})$. The combined organic extracts were washed with brine $(2 \times 150 \mathrm{~mL})$, dried $\left(\mathrm{Na}_{2} \mathrm{SO}_{4}\right)$, filtered, and concentrated by rotary evaporation. Purification by silica flash chromatography (10\% EtOAc in hexane) yielded the alkylated lactone 9 as a white solid (2.69 $\mathrm{g}, 65 \%$ yield $)$.

TLC: $\mathrm{R}_{\mathrm{f}} 0.35\left(10 \%\right.$ EtOAc in hexane, $\left.\mathrm{KMnO}_{4}\right) .{ }^{\mathbf{1}} \mathbf{H}-\mathbf{N M R}\left(600 \mathrm{MHz}, \mathrm{DMSO}-d_{6}\right): \delta 7.29-7.03$ $(\mathrm{m}, 8 \mathrm{H}), 6.52(\mathrm{~d}, J=7.1 \mathrm{~Hz}, 2 \mathrm{H}), 6.49$ (rotamer, d, $J=7.2 \mathrm{~Hz}), 6.27(\mathrm{~d}, J=3.1 \mathrm{~Hz}, 1 \mathrm{H}), 5.90$ (ddt, $J=16.8,10.2,6.1 \mathrm{~Hz}, 1 \mathrm{H}), 5.17(\mathrm{dd}, J=17.3,1.7 \mathrm{~Hz}, 1 \mathrm{H}), 5.10(\mathrm{~d}, J=3.2 \mathrm{~Hz}, 1 \mathrm{H}), 5.06$ $(\mathrm{dd}, J=10.2,2.0 \mathrm{~Hz}, 1 \mathrm{H}), 4.82(\mathrm{dd}, J=9.0,5.5 \mathrm{~Hz}, 1 \mathrm{H}), 4.73$ (rotamer, dd, $J=10.4,3.8 \mathrm{~Hz}$ ), $2.32-2.05(\mathrm{~m}, 4 \mathrm{H}), 1.39\left(\mathrm{~s}, 3 \mathrm{H}\right.$, rotamer from the Boc group), $1.02(\mathrm{~s}, 6 \mathrm{H})$. The ${ }^{1} \mathrm{H}-\mathrm{NMR}$ spectrum is consistent with the literature report ${ }^{5}$ except for the observation of rotamer peaks at $\mathrm{rt}$.<smiles>O=C1O[C@H](c2ccccc2)[C@H](c2ccccc2)N1CCC1CO1</smiles>

tert-Butyl (3S,5S,6R)-3-(2-((RS)oxiran-2-yl)ethyl)-2-oxo-5,6-diphenylmorpholine-4-

carboxylate (S3). Synthesized as previously described, with minor modifications. ${ }^{5}$ In a $100-\mathrm{mL}$ roundbottom flask, tert-butyl (3S,5S,6R)-3-(but-3-en-1-yl)-2-oxo-5,6-diphenylmorpholine-4carboxylate (9) (2.69 g, $6.60 \mathrm{mmol}, 1$ equiv) was dissolved in $\mathrm{CH}_{2} \mathrm{Cl}_{2}(15 \mathrm{~mL}, 0.44 \mathrm{M})$. The mixture was cooled to $0{ }^{\circ} \mathrm{C}$ and $m$-CPBA (77\% purity, $1.744 \mathrm{~g}, 8.581 \mathrm{mmol}, 1.3$ equiv) was added in one portion. A buffered phosphate solution $(\mathrm{pH} 7)(15 \mathrm{~mL})$ was added and the mixture was warmed to $\mathrm{rt}$ and stirred for $16 \mathrm{~h}$. Satd aq $\mathrm{NaHCO}_{3}(30 \mathrm{~mL})$ was added and the layers were

\footnotetext{
${ }^{4}$ Kasun, Z. A.; Gao, X.; Lipinski, R. M.; Krische, M. J. J. Am. Chem. Soc. 2015, 137 (28), 8900.

${ }^{5}$ Allevi, P.; Anastasia, M. Tetrahedron: Asymmetry 2004, 15 (13), 2091.
} 
separated. The aq layer was extracted with $\mathrm{CH}_{2} \mathrm{Cl}_{2}(3 \times 70 \mathrm{~mL})$ and the combined organic extracts were washed with brine $(2 \times 150 \mathrm{~mL})$, dried $\left(\mathrm{Na}_{2} \mathrm{SO}_{4}\right)$, filtered, and concentrated by rotary evaporation. Purification by silica flash chromatography (20\% EtOAc in hexanes) yielded the epoxide $\mathbf{S 3}$ as a white solid (2.43g, 87\% yield).

TLC: $\mathrm{R}_{\mathrm{f}} 0.30\left(20 \%\right.$ EtOAc in hexanes $\left.\mathrm{KMnO}_{4}\right) .{ }^{\mathbf{1}} \mathbf{H}-\mathbf{N M R}\left(500 \mathrm{MHz}, \mathrm{CDCl}_{3}\right): \delta 7.30-7.19(\mathrm{~m}$, $3 \mathrm{H}), 7.18-7.13(\mathrm{~m}, 1 \mathrm{H}), 7.08(\mathrm{td}, J=7.6,1.7 \mathrm{~Hz}, 2 \mathrm{H}), 7.02-6.92(\mathrm{~m}, 2 \mathrm{H}), 6.59$ (rotamer, d, $J$ $=8.1 \mathrm{~Hz}), 6.56(\mathrm{~d}, J=7.5 \mathrm{~Hz}, 2 \mathrm{H}), 5.94(\mathrm{~d}, J=2.9 \mathrm{~Hz}, 1 \mathrm{H}), 5.23$ (rotamer, d, $J=3.0 \mathrm{~Hz}), 5.08-$ $5.02(\mathrm{~m}, 1 \mathrm{H}), 5.01(\mathrm{~d}, J=3.0 \mathrm{~Hz}, 1 \mathrm{H}), 4.85$ (rotamer, ddd, $J=26.2,10.6,4.2 \mathrm{~Hz}$ ), 3.05 (ddd, $J$ $=7.8,3.9,2.0 \mathrm{~Hz}, 1 \mathrm{H}), 2.86-2.73(\mathrm{~m}, 1 \mathrm{H}), 2.60(\mathrm{ddd}, J=11.2,4.9,2.6 \mathrm{~Hz}, 1 \mathrm{H}), 2.47-2.23$ $(\mathrm{m}, 1 \mathrm{H}), 2.23-1.90(\mathrm{~m}, 2 \mathrm{H}), 1.88-1.51(\mathrm{~m}, 1 \mathrm{H}), 1.46$ (rotamer of one diastereomer, $\mathrm{s}, 3 \mathrm{H})$, 1.45 (rotamer of the other diastereomer, $\mathrm{s}, 3 \mathrm{H}), 1.10(\mathrm{~s}, 6 \mathrm{H}$, one diastereomer), $1.09(\mathrm{~s}, 6 \mathrm{H}$, the other diastereomer). The ${ }^{1} \mathrm{H}-\mathrm{NMR}$ spectrum is consistent with the literature report. ${ }^{5}$ A $1: 1 \mathrm{dr}$ was determined based on integration of the signals corresponding to the Boc groups.<smiles>N[C@@H](O)CC[C@H]1C(=O)O[C@@H](c2ccccc2)[C@H](c2ccccc2)N1C(=O)O</smiles>

(2L,5S)-8<smiles>N#C[C@H](O)CC[C@H]1N[C@@H](c2ccccc2)[C@H](c2ccccc2)OC1=O</smiles>

$(2\llcorner, 5 R)-8$

tert-Butyl (3S,5S,6R)-3-((S)4-azido-3-hydroxybutyl)-2-oxo-5,6-diphenylmorpholine-4carboxylate $((2 \mathrm{~L}, 5 S)-8)$ and tert-butyl $(3 S, 5 S, 6 R)-3-((R) 4$-azido-3-hydroxybutyl)-2-oxo-5,6diphenylmorpholine-4-carboxylate $((\mathbf{2} \mathbf{L}, \mathbf{5} R)-8)$. Synthesized as previously described, with minor modifications. ${ }^{5}$ In a $100-\mathrm{mL}$ roundbottom flask, tert-butyl $(3 S, 5 S, 6 R)-3-(2-((R S)$ oxiran-2yl)ethyl)-2-oxo-5,6-diphenylmorpholine-4-carboxylate (S3) (1:1 dr, $4.73 \mathrm{~g}, 11.16 \mathrm{mmol}, 1.0$ equiv) was dissolved in 2-methoxyethanol (40 mL, $0.28 \mathrm{M})$, and $\mathrm{NaN}_{3}(2.03 \mathrm{~g}, 31.24$ mmol, 2.8 equiv) and $\mathrm{NH}_{4} \mathrm{Cl}$ (1.31 g, $24.55 \mathrm{mmol}, 2.2$ equiv) were added. The flask was then fitted with a condenser and heated to $50{ }^{\circ} \mathrm{C}$ for $12 \mathrm{~h}$. The mixture was cooled to $\mathrm{rt}$ and $\mathrm{H}_{2} \mathrm{O}(100 \mathrm{~mL})$ and $\mathrm{CH}_{2} \mathrm{Cl}_{2}(100 \mathrm{~mL})$ were added and the layers were separated. The aq layer was extracted with $\mathrm{CH}_{2} \mathrm{Cl}_{2}(3 \times 60 \mathrm{~mL})$. The combined organic extracts were washed with large amount of water to remove $\mathrm{NaN}_{3}$, dried $\left(\mathrm{Na}_{2} \mathrm{SO}_{4}\right)$, filtered, and concentrated by rotary evaporation to afford the crude product as a white foamy solid. Purification by silica flash chromatography (1\% EtOAc in $\mathrm{CH}_{2} \mathrm{Cl}_{2}$ ) afforded the individual diastereomeric azidoalcohols (2L,5S)-8 (1.18 g, 23\%) and (2L,5R)-8 (1.37 g, 26\%), in addition to mixed fractions (1.30 g, 25\%), as white solids for an overall combined yield of $74 \%$.<smiles>N#C[C@H](O)CC[C@H]1C(=O)O[C@@H](c2ccccc2)[C@H](c2ccccc2)N1C(=O)O</smiles>

tert-Butyl (3S,5S,6R)-3-((S)-4-azido-3-hydroxybutyl)-2-oxo-5,6-diphenylmorpholine-4-carboxylate $((2 \mathrm{~L}, 5 S)-8)$. The ${ }^{1} \mathrm{H}-\mathrm{NMR}$ of this compound has been reported in the literature. ${ }^{5}$

TLC: $\mathrm{R}_{\mathrm{f}} 0.30\left(5 \%\right.$ EtOAc in $\left.\mathrm{CH}_{2} \mathrm{Cl}_{2}, \mathrm{KMnO}_{4}\right)$. mp: $107-108^{\circ} \mathrm{C}$. Optical Rotation: $[\alpha]_{D}^{1 /}$ : $-56.7^{\circ}$ (c 0.50, $\left.\mathrm{CHCl}_{3}\right)$. IR (ZnSe, film): 3448 (br), 2977 (w), 2930 (w), 2102 (s), 1756 (s), 1697 (s), 1454 (m), 1392 (m), 1367 (m), 1274 (m), $1164(\mathrm{~m}) \mathrm{cm}^{-1}$. ${ }^{1}$ H-NMR (600 MHz, CDCl $)$ : 
$\delta 7.28-7.20(\mathrm{~m}, 3 \mathrm{H}), 7.19-7.13(\mathrm{~m}, 1 \mathrm{H}), 7.08(\mathrm{t}, J=7.6 \mathrm{~Hz}, 2 \mathrm{H}), 7.01-6.93(\mathrm{~m}, 2 \mathrm{H}), 6.58$ (rotamer, d, $J=7.7 \mathrm{~Hz}$ ), $6.55(\mathrm{~d}, J=7.2 \mathrm{~Hz}, 2 \mathrm{H}), 5.93$ (rotamer, d, $J=3.0 \mathrm{~Hz}), 5.92(\mathrm{~d}, J=3.1$ $\mathrm{Hz}, 1 \mathrm{H}), 5.23$ (rotamer, d, $J=3.0 \mathrm{~Hz}$ ), $5.08(\mathrm{dd}, J=9.7,5.1 \mathrm{~Hz}, 1 \mathrm{H}), 5.00(\mathrm{~d}, J=3.1 \mathrm{~Hz}, 1 \mathrm{H})$, 4.87 (rotamer, dd, $J=10.6,4.2 \mathrm{~Hz}$ ), 3.94 (dq, $J=8.1,4.0 \mathrm{~Hz}, 1 \mathrm{H}$ ), 3.82 (rotamer, dq, $J=8.6,4.0$ $\mathrm{Hz}), 3.48-3.22(\mathrm{~m}, 2 \mathrm{H}), 2.83(\mathrm{~d}, J=4.8 \mathrm{~Hz}, 1 \mathrm{H}), 2.61-2.40$ (rotamer, m), $2.37-2.27(\mathrm{~m}, 1 \mathrm{H})$, $2.16-2.06(\mathrm{~m}, 1 \mathrm{H}), 2.03-1.95$ (rotamer, $\mathrm{m}), 1.87-1.78(\mathrm{~m}, 2 \mathrm{H}), 1.76-1.68$ (rotamer, $\mathrm{m}$ ), 1.46 (rotamer, s, 3H), $1.10(\mathrm{~s}, 6 \mathrm{H}) .{ }^{13} \mathbf{C}-\mathbf{N M R}\left(150 \mathrm{MHz}, \mathrm{CDCl}_{3}\right): \delta 169.5,154.0,136.3,134.3$, 128.8, 128.3, 127.9, 127.5, 126.6, 81.7, 79.2, 70.9, 61.6, 57.1, 56.1, 32.0, 30.4, 28.0. ESI-MS: Calcd for $\mathrm{C}_{25} \mathrm{H}_{31} \mathrm{~N}_{4} \mathrm{O}_{5}\left([\mathrm{M}+\mathrm{H}]^{+}\right)$: 467.2294, Found: 467.2272 .

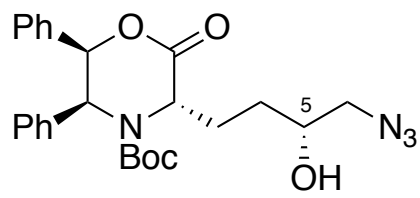

tert-Butyl (3S,5S,6R)-3-((R)-4-azido-3-hydroxybutyl)-2-oxo-5,6-diphenylmorpholine-4-carboxylate $((2 \mathrm{~L}, \mathbf{5} \boldsymbol{R})-\mathbf{8})$. The ${ }^{1} \mathrm{H}-\mathrm{NMR}$ of this compound has been reported in the literature. ${ }^{5}$

TLC: $\mathrm{R}_{\mathrm{f}} 0.40\left(5 \%\right.$ EtOAc in $\left.\mathrm{CH}_{2} \mathrm{Cl}_{2}, \mathrm{KMnO}_{4}\right)$. mp: $110-111^{\circ} \mathrm{C}$. Optical Rotation: $[\alpha]_{D}^{17}$ : $-43.7^{\circ}$ ( c 0.50, $\mathrm{CHCl}_{3}$ ). IR (ZnSe, film): 3369 (br), 2967 (w), 2927 (w), 2099 (s), 1748 (s), 1698 (s), $1681(\mathrm{~s}), 1526(\mathrm{w}), 1454(\mathrm{~m}), 1256(\mathrm{~m}), 1162(\mathrm{~s}) \mathrm{cm}^{-1} .{ }^{1}$ H-NMR $\left(600 \mathrm{MHz}, \mathrm{CDCl}_{3}\right):(\approx 5: 1$ mixture of rotamers; peaks for minor rotamer annotated only for the Boc group) $\delta 7.30-7.20$ $(\mathrm{m}, 3 \mathrm{H}), 7.19-7.14(\mathrm{~m}, 1 \mathrm{H}), 7.09(\mathrm{t}, J=7.6 \mathrm{~Hz}, 2 \mathrm{H}), 7.01-6.96(\mathrm{~m}, 2 \mathrm{H}), 6.56(\mathrm{~d}, J=7.2 \mathrm{~Hz}$, 2H), $5.91(\mathrm{~d}, J=3.0 \mathrm{~Hz}, 1 \mathrm{H}), 5.00(\mathrm{~d}, J=2.8 \mathrm{~Hz}, 1 \mathrm{H}), 5.00-4.97(\mathrm{~m}, 1 \mathrm{H}), 4.20(\mathrm{tt}, J=7.2,3.7$ $\mathrm{Hz}, 1 \mathrm{H}), 3.47$ (d, $J=4.4 \mathrm{~Hz}, 1 \mathrm{H}), 3.40(\mathrm{dd}, J=12.5,3.4 \mathrm{~Hz}, 1 \mathrm{H}), 3.32(\mathrm{dd}, J=12.5,7.1 \mathrm{~Hz}$, $1 \mathrm{H}$ ), 2.33 (dddd, $J=13.7,10.3,6.8,3.7 \mathrm{~Hz}, 1 \mathrm{H}), 2.16$ (dddd, $J=17.5,11.0,6.5,4.4 \mathrm{~Hz}, 1 \mathrm{H}$ ), 1.81 (dddd, $J=13.3,9.8,6.5,3.2 \mathrm{~Hz}, 1 \mathrm{H}$ ), 1.71 (dtd, $J=14.2,6.5,3.4 \mathrm{~Hz}, 1 \mathrm{H}), 1.46$ (rotamer, s), $1.12(\mathrm{~s}, 9 \mathrm{H}) .{ }^{13} \mathbf{C}-\mathbf{N M R}\left(150 \mathrm{MHz}, \mathrm{CDCl}_{3}\right): \delta 169.1,154.1,136.0,134.16,128.8,128.3$, 127.9, 127.5, 126.6, 82.1, 79.4, 68.7, 61.4, 57.0, 54.7, 31.0, 29.8, 28.5 (rotamer), 28.0. ESI-MS: Calcd for $\mathrm{C}_{25} \mathrm{H}_{30} \mathrm{~N}_{4} \mathrm{O}_{5} \mathrm{Na}\left([\mathrm{M}+\mathrm{Na}]^{+}\right)$: 489.2114, Found: 489.2115 . 


\section{E. SYNTHESIS OF SF2768 (2L,5S-1) AND 5-epi-SF2768 (2L,5R-1)}<smiles>NCC(O)CC[C@H]1C(=O)OC(c2ccccc2)C(c2ccccc2)[C@H]1c1ccccc1</smiles>

(2L,5S)-8 (shown) $(2 \mathrm{~L}, 5 R)-8$

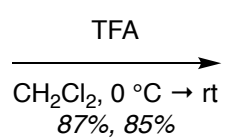

$87 \%, 85 \%$

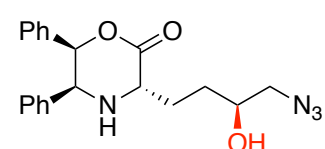

(2L,5S)-13 (shown) $(2 L, 5 R)-13$
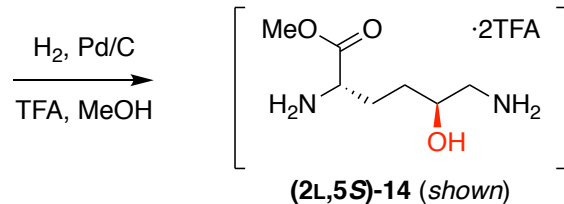
$(2 L, 5 R)-14$
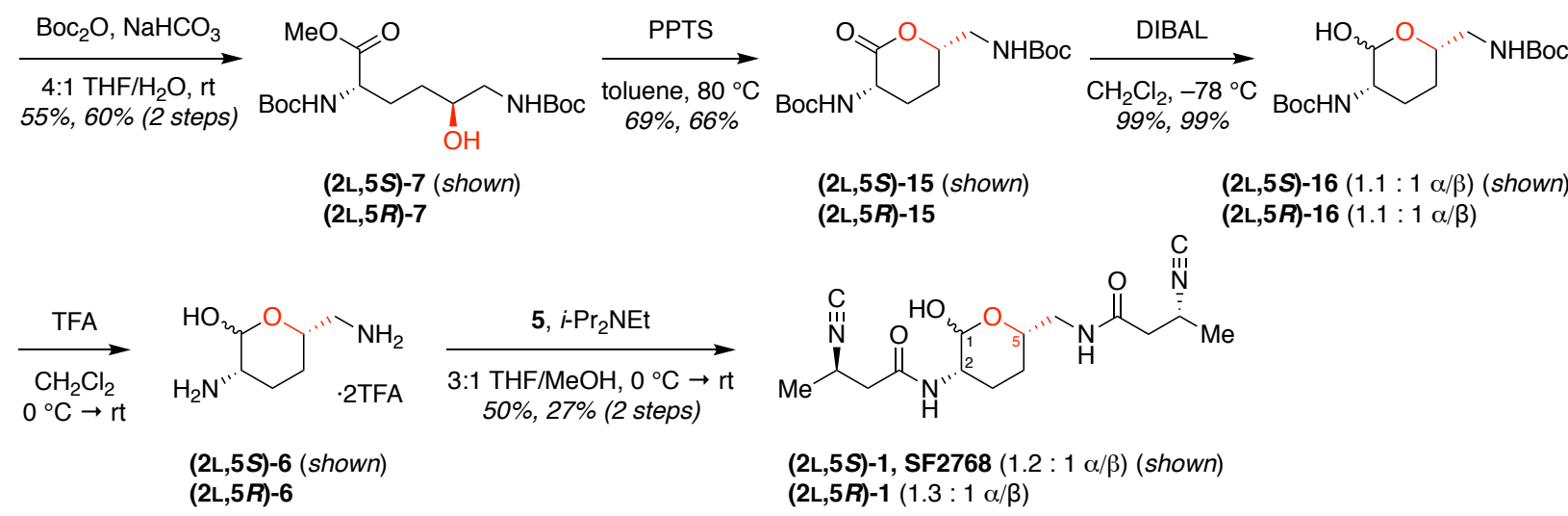

Figure S4. Synthesis of SF2768 (2L,5S-1) and 5-epi-SF2768 (2L-5R)-1.<smiles></smiles>

(3S,5S,6R)-3-((S)-4-Azido-3-hydroxybutyl)-5,6-diphenylmorpholin-2-one ((2L,5S)-13). In a 25-mL roundbottom flask, tert-butyl (3S,5S,6R)-3-((S)-4-azido-3-hydroxybutyl)-2-oxo-5,6diphenylmorpholine-4-carboxylate $((2 \mathrm{~L}, 5 S)-8)(0.964 \mathrm{~g}, 2.07 \mathrm{mmol}, 1.0$ equiv) was dissolved in $\mathrm{CH}_{2} \mathrm{Cl}_{2}(5.5 \mathrm{~mL}, 0.37 \mathrm{M})$. The mixture was cooled to $0{ }^{\circ} \mathrm{C}$ and TFA $(2.35 \mathrm{~g}, 20.7 \mathrm{mmol}, 10$ equiv) was added dropwise. The mixture was warmed to rt and stirred for $2 \mathrm{~h}$. The mixture was concentrated by rotary evaporation, redissolved in $\mathrm{CH}_{2} \mathrm{Cl}_{2}(50 \mathrm{~mL})$, and satd aq $\mathrm{NaHCO}_{3}(100$ $\mathrm{mL}$ ) and stirred vigorously until bubbling ceased. The mixture was transferred into a separatory funnel and layers were separated and the aq layer extracted with $\mathrm{CH}_{2} \mathrm{Cl}_{2}(3 \mathrm{x} 50 \mathrm{~mL})$. The combined organic extracts were washed with brine $(2 \times 100 \mathrm{~mL})$, dried $\left(\mathrm{Na}_{2} \mathrm{SO}_{4}\right)$, filtered, and concentrated by rotary evaporation. Purification by silica flash chromatography $(1 \% \mathrm{MeOH}$ in $\mathrm{CH}_{2} \mathrm{Cl}_{2}$ ) yielded the amine $(\mathbf{2} \mathbf{L}, \mathbf{5 S})-\mathbf{1 3}$ as a white solid $(0.661 \mathrm{~g}, 87 \%$ yield $)$.

TLC: $\mathrm{R}_{\mathrm{f}} 0.40\left(5 \% \mathrm{MeOH}\right.$ in $\left.\mathrm{CH}_{2} \mathrm{Cl}_{2}, \mathrm{KMnO}_{4}\right)$. mp: $102-103{ }^{\circ} \mathrm{C}$. Optical Rotation: $[\alpha]_{\mathrm{D}}{ }^{17}$ : $58.3^{\circ}$ (c 1.0, $\left.\mathrm{CHCl}_{3}\right)$. IR (ZnSe, film): 3429 (br), 2927 (w), 2103 (s), 1741 (s), 1453 (m), 1291 (w), $1197(\mathrm{~m}), 1065(\mathrm{~m}) \mathrm{cm}^{-1}$. ${ }^{1} \mathbf{H}-\mathbf{N M R}\left(600 \mathrm{MHz}, \mathrm{CDCl}_{3}\right): \delta 7.28-7.24(\mathrm{~m}, 3 \mathrm{H}), 7.23-7.19$ $(\mathrm{m}, 3 \mathrm{H}), 7.04(\mathrm{dd}, J=6.6,2.9 \mathrm{~Hz}, 2 \mathrm{H}), 7.01(\mathrm{dd}, J=6.6,2.9 \mathrm{~Hz}, 2 \mathrm{H}), 4.89(\mathrm{t}, J=5.2 \mathrm{~Hz}, 1 \mathrm{H})$, $4.31-4.18(\mathrm{~m}, 1 \mathrm{H}), 4.04(\mathrm{~d}, J=5.2 \mathrm{~Hz}, 1 \mathrm{H}), 3.42(\mathrm{dd}, J=13.2,5.9 \mathrm{~Hz}, 1 \mathrm{H}), 3.37(\mathrm{dd}, J=13.1$, $4.4 \mathrm{~Hz}, 1 \mathrm{H}), 3.23$ (dd, $J=10.5,8.1 \mathrm{~Hz}, 2 \mathrm{H}), 2.71(\mathrm{~s}, 1 \mathrm{H}), 2.24$ (dddd, $J=13.5,9.7,8.1,6.6 \mathrm{~Hz}$, $1 \mathrm{H}), 1.91-1.81(\mathrm{~m}, 1 \mathrm{H}), 1.78(\mathrm{ddd}, J=14.4,10.4,6.6 \mathrm{~Hz}, 1 \mathrm{H}), 1.63(\mathrm{dtd}, J=13.4,10.1,5.3$ $\mathrm{Hz}, 1 \mathrm{H}) .{ }^{13} \mathbf{C}-\mathbf{N M R}\left(151 \mathrm{MHz}, \mathrm{CDCl}_{3}\right): \delta 173.4,140.0,138.1,128.6,128.0,127.8,126.8,76.6$, 75.5, 65.5, 53.9, 52.0, 24.3, 23.4. ESI-MS: Calcd for $\mathrm{C}_{20} \mathrm{H}_{23} \mathrm{~N}_{4} \mathrm{O}_{3}\left([\mathrm{M}+\mathrm{H}]^{+}\right)$: 367.1770 , Found: 367.1753 . 
<smiles>N#[N+]C[C@H](O)CC[C@H]1NC(c2ccccc2)[C@H](c2ccccc2)OC1=O</smiles>

(3S,5S,6R)-3-((R)-4-azido-3-hydroxybutyl)-5,6-diphenylmorpholin-2-one ((2L,5R)-13). Synthesized by the procedure above from $(2 \mathrm{~L}, 5 \boldsymbol{R})-8$ as a white solid $(0.646 \mathrm{~g}, 85 \%$ yield $)$.

TLC: $\mathrm{R}_{\mathrm{f}} 0.40\left(5 \% \mathrm{MeOH}\right.$ in $\left.\mathrm{CH}_{2} \mathrm{Cl}_{2}, \mathrm{KMnO}_{4}\right)$. mp: $137-138{ }^{\circ} \mathrm{C}$. Optical Rotation: $[\alpha]_{D}^{17}$ : 29.0 (c 1.00, $\mathrm{CHCl}_{3}$ ). IR (ZnSe, film): 3309 (w), 3174 (br), 2916 (w), 2106 (s), 1727 (s), 1456 $(\mathrm{w}), 1234(\mathrm{~m}), 1199(\mathrm{~m}), 1056(\mathrm{~m}) \mathrm{cm}^{-1}$. ${ }^{1} \mathbf{H}-\mathbf{N M R}\left(500 \mathrm{MHz}, \mathrm{CDCl}_{3}\right): \delta 7.24(\mathrm{~d}, J=4.7 \mathrm{~Hz}$, $3 \mathrm{H}), 7.18(\mathrm{dd}, J=5.1,2.0 \mathrm{~Hz}, 3 \mathrm{H}), 6.99(\mathrm{dd}, J=6.6,3.0 \mathrm{~Hz}, 2 \mathrm{H}), 6.92$ (dd, $J=6.8,2.9 \mathrm{~Hz}, 2 \mathrm{H})$, 4.89 (d, $J=3.9 \mathrm{~Hz}, 1 \mathrm{H}), 4.42$ (dt, $J=7.3,4.0 \mathrm{~Hz}, 1 \mathrm{H}), 4.07$ (d, $J=4.9 \mathrm{~Hz}, 1 \mathrm{H}), 3.45$ (dd, $J=$ 13.1, $4.3 \mathrm{~Hz}, 1 \mathrm{H}), 3.33(\mathrm{dd}, J=13.1,4.8 \mathrm{~Hz}, 1 \mathrm{H}), 3.22(\mathrm{~d}, J=6.3 \mathrm{~Hz}, 1 \mathrm{H}), 3.14-3.03(\mathrm{~m}, 1 \mathrm{H})$, 2.97 (br, 1H), $2.27-2.06(\mathrm{~m}, 1 \mathrm{H}), 1.89-1.79(\mathrm{~m}, 1 \mathrm{H}), 1.75-1.50(\mathrm{~m}, 2 \mathrm{H}) .{ }^{13} \mathbf{C}-\mathbf{N M R}(125$ $\left.\mathrm{MHz}, \mathrm{CDCl}_{3}\right): \delta 171.8,139.9,137.6,128.6,128.0,127.7,126.8,79.7,76.7,64.9,54.5,54.6$, 26.3, 25.1. ESI-MS: Calcd for $\mathrm{C}_{20} \mathrm{H}_{23} \mathrm{~N}_{4} \mathrm{O}_{3}\left([\mathrm{M}+\mathrm{H}]^{+}\right): 367.1770$, Found: 367.1765 .<smiles>COC(=O)[C@H](CC[C@H](O)CNC(=O)OCc1ccccc1)C(=O)OCc1ccccc1</smiles>

Methyl $(2 S, 5 S)$-2,6-bis((tert-butoxycarbonyl)amino)-5-hydroxyhexanoate $((2 \mathrm{~L}, 5 S)-7)$. In a 25 $\mathrm{mL}$ roundbottom flask were placed $(3 S, 5 S, 6 R)-3-((S)$-4-azido-3-hydroxybutyl)-5,6-diphenylmorpholin-2-one ((2L,5S)-13) (104 mg, $0.284 \mathrm{mmol}, 1$ equiv) and 10\% Pd/C (104 mg, 100\% $\mathrm{w} / \mathrm{w})$ followed by addition of anhydrous $\mathrm{MeOH}(10 \mathrm{~mL}, 10 \mathrm{mg} / \mathrm{mL})$. TFA $(0.109 \mathrm{~mL}, 5$ equiv) was added dropwise and the mixture was stirred under $\mathrm{H}_{2}$ atmosphere (balloon pressure) at $\mathrm{rt}$ for $3 \mathrm{~h}$. The solution was filtered through Celite and the filter cake was washed with $\mathrm{MeOH}$. The combined filtrates were concentrated by rotary evaporation, then taken up in $\mathrm{H}_{2} \mathrm{O}(20 \mathrm{~mL})$ and EtOAc $(20 \mathrm{~mL})$, and the aq phase was collected. Lyophilization afforded the crude amine TFA salt $(\mathbf{2} \mathbf{L}, \mathbf{5 S})-\mathbf{1 4},{ }^{6}$ which was carried forward without further purification.

In a $25 \mathrm{~mL}$ roundbottom flask, the amine TFA salt ((2L,5S)-14) above (114 mg, $0.282 \mathrm{mmol}, 1.0$ equiv) was dissolved in THF $(1.5 \mathrm{~mL})$ and water $(0.5 \mathrm{~mL})$. The mixture was cooled to $0{ }^{\circ} \mathrm{C}$, then solid $\mathrm{NaHCO}_{3}(54.5 \mathrm{mg}, 0.648 \mathrm{mmol}, 2.3$ equiv) and di-tert-butyl dicarbonate $(1.0 \mathrm{M}$ in THF) (0.648 mL, $0.648 \mathrm{mmol}, 2.3$ equiv) were added. The mixture was warmed to rt and stirred for $2 \mathrm{~h}$. The solvent was removed by rotary evaporation and the residue taken up in $\mathrm{CH}_{2} \mathrm{Cl}_{2}(30$ $\mathrm{mL})$ and water $(30 \mathrm{~mL})$. The layers were separated and the aq layer extracted with $\mathrm{CH}_{2} \mathrm{Cl}_{2}(2 \mathrm{x}$ $20 \mathrm{~mL})$. The combined organic extracts were dried $\left(\mathrm{Na}_{2} \mathrm{SO}_{4}\right)$, filtered, and concentrated by rotary evaporation. Purification by silica flash chromatography $\left(10 \%-30 \%\right.$ EtOAc in $\left.\mathrm{CH}_{2} \mathrm{Cl}_{2}\right)$ yielded the bis-Boc diamine (2L,5S)-7 as a yellow oil (58.8 mg, 55\% yield over two steps).

\footnotetext{
${ }^{6}$ The crude mixture included $\approx 20 \%$ byproduct (see SECTION L for ${ }^{1}$ H-NMR of crude (2L,5S)-14). The reaction should be monitored by LC-MS if changing the reaction concentration or scale. Prolonging the reaction time may favor intramolecular lactam formation. Shortening the reaction time may not afford the methyl ester product as the major product.
} 
TLC: $\mathrm{R}_{\mathrm{f}} 0.35\left(30 \%\right.$ EtOAc in $\left.\mathrm{CH}_{2} \mathrm{Cl}_{2}, \mathrm{KMnO}_{4}\right)$. Optical Rotation: $[\alpha]_{\mathrm{D}}{ }^{20}: 11.6^{\circ}(c 1.00$, $\mathrm{CHCl}_{3}$ ). IR (ZnSe, film): 3368 (br), 2978 (m), 2930 (w), 1740 (m), 1690 (s), 1515 (m), 1366 $(\mathrm{m}), 1250(\mathrm{~m}), 1164(\mathrm{~s}) \mathrm{cm}^{-1} .{ }^{1} \mathbf{H}-\mathbf{N M R}\left(600 \mathrm{MHz}, \mathrm{CDCl}_{3}\right): \delta 5.18(\mathrm{~d}, J=8.2 \mathrm{~Hz}, 1 \mathrm{H}), 5.02-$ $4.91(\mathrm{~m}, 1 \mathrm{H}), 4.32(\mathrm{td}, J=7.1,6.4 \mathrm{~Hz}, 1 \mathrm{H}), 3.73(\mathrm{~s}, 3 \mathrm{H}), 3.72-3.67(\mathrm{~m}, 1 \mathrm{H}), 3.26(\mathrm{tdd}, J=$ $12.4,7.9,5.5 \mathrm{~Hz}, 1 \mathrm{H}), 3.04$ (dt, $J=14.1,6.4 \mathrm{~Hz}, 1 \mathrm{H}), 2.89$ (br, $1 \mathrm{H}), 1.90$ (ddd, $J=10.6,6.3,5.5$ $\mathrm{Hz}, 1 \mathrm{H}), 1.85-1.73(\mathrm{~m}, 1 \mathrm{H}), 1.52$ (tdd, $J=10.4,5.7,3.4 \mathrm{~Hz}, 1 \mathrm{H}), 1.49-1.44(\mathrm{~m}, 1 \mathrm{H}), 1.43$ (s, 18H). ${ }^{13}$ C-NMR $\left(126 \mathrm{MHz}, \mathrm{CDCl}_{3}\right): \delta 173.4,157.1,155.7,80.0,79.8,70.9,53.3,52.4,46.7$, 30.3, 28.8, 28.5. ESI-MS: Calcd for $\mathrm{C}_{17} \mathrm{H}_{33} \mathrm{~N}_{2} \mathrm{O}_{7}\left([\mathrm{M}+\mathrm{H}]^{+}\right)$: 377.2288, Found: 377.2285.

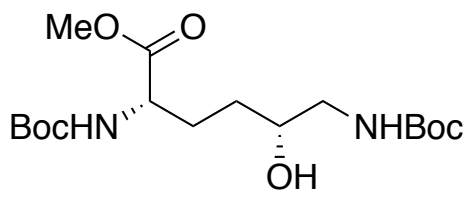

Methyl (2S,5R)-2,6-bis((tert-butoxycarbonyl)amino)-5-hydroxyhexanoate $((2 \mathrm{~L}, 5 R)-7)$ :

Synthesized by the procedure above from $(\mathbf{2 L}, \mathbf{5 R})-\mathbf{1 3}$ as a light yellow oil $(64.1 \mathrm{mg}, 60 \%$ yield over two steps).

TLC: $\mathrm{R}_{\mathrm{f}} 0.35\left(30 \%\right.$ EtOAc in $\left.\mathrm{CH}_{2} \mathrm{Cl}_{2}, \mathrm{KMnO}_{4}\right)$. Optical Rotation: $[\alpha]_{\mathrm{D}}{ }^{20}: 6.79^{\circ}(c) 1.00$, $\mathrm{CHCl}_{3}$ ). IR (ZnSe, film): 3366 (br), 2978 (m), 2930 (m), 1742 (m), 1696 (s), 1521 (m), 1367 $(\mathrm{m}), 1252(\mathrm{~m}), 1170(\mathrm{~s}) \mathrm{cm}^{-1} .{ }^{1} \mathbf{H}-\mathbf{N M R}\left(600 \mathrm{MHz}, \mathrm{CDCl}_{3}\right): \delta 5.27(\mathrm{~d}, J=8.0 \mathrm{~Hz}, 1 \mathrm{H}), 5.01(\mathrm{~d}$, $J=6.6 \mathrm{~Hz}, 1 \mathrm{H}), 4.33(\mathrm{q}, J=7.2 \mathrm{~Hz}, 1 \mathrm{H}), 3.73(\mathrm{~s}, 3 \mathrm{H}), 3.72-3.64(\mathrm{~m}, 1 \mathrm{H}), 3.27(\mathrm{dt}, J=10.6$, $5.1 \mathrm{~Hz}, 1 \mathrm{H}), 3.20-3.06(\mathrm{br}, 1 \mathrm{H}), 3.01(\mathrm{dt}, J=13.7,6.3 \mathrm{~Hz}, 1 \mathrm{H}), 1.98(\mathrm{td}, J=8.4,4.4 \mathrm{~Hz}, 1 \mathrm{H})$, $1.70(\mathrm{dtd}, J=14.2,8.5,6.1 \mathrm{~Hz}, 1 \mathrm{H}), 1.56-1.45(\mathrm{~m}, 2 \mathrm{H}), 1.43(\mathrm{~s}, 18 \mathrm{H}) .{ }^{13} \mathrm{C}-\mathbf{N M R}(151 \mathrm{MHz}$, $\left.\mathrm{CDCl}_{3}\right): \delta 173.3,157.0,155.9,80.3,79.8,71.2,53.2,52.5,46.8,30.3,29.7,28.5,28.4$. ESI-MS: Calcd for $\mathrm{C}_{17} \mathrm{H}_{33} \mathrm{~N}_{2} \mathrm{O}_{7}\left([\mathrm{M}+\mathrm{H}]^{+}\right): 377.2288$, Found: 377.2282 .

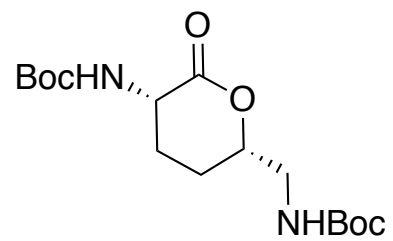

tert-Butyl (((2S,5S)-5-((tert-butoxycarbonyl)amino)-6-oxotetrahydro-2H-pyran-2-yl)methyl)carbamate $((\mathbf{2}, \mathbf{5 S})-\mathbf{1 5})$. Synthesized by a modified version of the general protocol reported in the literature. ${ }^{7}$ In a $25 \mathrm{~mL}$ roundbottom flask, methyl (2S,5S)-2,6-bis((tertbutoxycarbonyl)amino)-5-hydroxyhexanoate $((2 \mathbf{L}, 5 S)-7)(58.8 \mathrm{mg}, 0.156 \mathrm{mmol}, 1$ equiv) and pyridinium $p$-toluenesulfonate $(7.85 \mathrm{mg}, 0.031 \mathrm{mmol}, 0.2$ equiv) were dissolved in dry toluene $(2.5 \mathrm{~mL}, 62 \mathrm{mM})$. The mixture was heated to $80{ }^{\circ} \mathrm{C}$ for $12 \mathrm{~h}$, then cooled to $\mathrm{rt}$ and concentrated by rotary evaporation. Purification by silica flash chromatography (20-40\% EtOAc in hexanes) yielded lactone (2L,5S)-15 as a white solid (37.1 $\mathrm{mg}, 69 \%$ yield).

TLC: $\mathrm{R}_{\mathrm{f}} 0.30\left(40 \%\right.$ EtOAc in hexanes, $\left.\mathrm{KMnO}_{4}\right)$. mp: $111-112{ }^{\circ} \mathrm{C}$. Optical Rotation: $[\alpha]_{\mathrm{D}}{ }^{20}$ : $70.6^{\circ}$ (c 1.00, $\left.\mathrm{CHCl}_{3}\right)$. IR (ZnSe, film): 3348 (br), 2978 (m), 2933 (m), 1751 (m), 1693 (s), 1515 (s), $1366(\mathrm{~m}), 1248(\mathrm{~m}), 1161(\mathrm{~s}) \mathrm{cm}^{-1}$. ${ }^{1} \mathbf{H}-\mathbf{N M R}\left(600 \mathrm{MHz}, \mathrm{CDCl}_{3}\right): \delta 5.33(\mathrm{~d}, J=6.6 \mathrm{~Hz}, 1 \mathrm{H})$, $4.99(\mathrm{t}, J=6.1 \mathrm{~Hz}, 1 \mathrm{H}), 4.53-4.35(\mathrm{~m}, 2 \mathrm{H}), 3.54-3.45(\mathrm{~m}, 1 \mathrm{H}), 3.18(\mathrm{dt}, J=13.9,6.3 \mathrm{~Hz}$, $1 \mathrm{H}), 2.67-2.50(\mathrm{~m}, 1 \mathrm{H}), 2.00$ (ddt, $J=15.0,10.2,4.3 \mathrm{~Hz}, 1 \mathrm{H}), 1.74$ (dtd, $J=16.5,11.6,5.4 \mathrm{~Hz}$,

\footnotetext{
${ }^{7}$ Zhu, W.; Ma, D. Org. Lett. 2003, 5 (26), 5063.
} 
1H), 1.55 (ddt, $J=16.8,12.0,5.0 \mathrm{~Hz}, 1 \mathrm{H}), 1.43(\mathrm{~s}, 9 \mathrm{H}), 1.42(\mathrm{~s}, 9 \mathrm{H}) .{ }^{13} \mathrm{C}-\mathbf{N M R}(151 \mathrm{MHz}$, $\left.\mathrm{CDCl}_{3}\right): \delta 172.9,156.0,155.3,80.4,80.0,76.9,48.6,44.0,28.5,28.4,24.7,23.3$. ESI-MS: Calcd for $\mathrm{C}_{16} \mathrm{H}_{28} \mathrm{~N}_{2} \mathrm{O}_{6} \mathrm{Na}\left([\mathrm{M}+\mathrm{Na}]^{+}\right)$: 367.1845 , Found: 367.1843 .

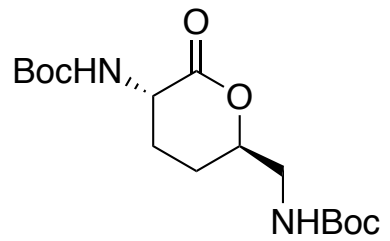

tert-Butyl (((2R,5S)-5-((tert-butoxycarbonyl)amino)-6-oxotetrahydro-2H-pyran-2-yl)methyl)carbamate $((\mathbf{2}, \mathbf{5 R})-\mathbf{1 5})$. Synthesized by the procedure above from $(\mathbf{2} \mathrm{L}, \mathbf{5} R)-7$ as a light yellow oil (38.7 mg, 66\% yield).

TLC: $\mathrm{R}_{\mathrm{f}} 0.30\left(40 \%\right.$ EtOAc in hexanes, $\left.\mathrm{KMnO}_{4}\right)$. Optical Rotation: $[\alpha]_{\mathrm{D}}{ }^{20}:-9.75^{\circ}(c 1.00$, $\mathrm{CHCl}_{3}$ ). IR (ZnSe, film): 3350 (br), 2978 (m), 2932 (m), 1743 (m), 1694 (s), 1514 (s), 1366 (m), $1240(\mathrm{~m}), 1160(\mathrm{~s}) \mathrm{cm}^{-1}$. ${ }^{1} \mathbf{H}-\mathbf{N M R}\left(600 \mathrm{MHz}, \mathrm{CDCl}_{3}\right): \delta 5.32-5.19(\mathrm{~m}, 1 \mathrm{H}), 5.05-4.90(\mathrm{~m}$, $1 \mathrm{H}), 4.51-4.39(\mathrm{~m}, 1 \mathrm{H}), 4.14-3.98(\mathrm{~m}, 1 \mathrm{H}), 3.53-3.45(\mathrm{~m}, 1 \mathrm{H}), 3.21(\mathrm{dt}, J=14.6,6.2 \mathrm{~Hz}$, $1 \mathrm{H}), 2.48-2.33(\mathrm{~m}, 1 \mathrm{H}), 1.99(\mathrm{dq}, J=10.2,3.4 \mathrm{~Hz}, 1 \mathrm{H}), 1.90-1.82(\mathrm{~m}, 1 \mathrm{H}), 1.81-1.72(\mathrm{~m}$, 1H), $1.44(\mathrm{~s}, 9 \mathrm{H}), 1.43(\mathrm{~s}, 9 \mathrm{H}) .{ }^{13} \mathbf{C}-\mathbf{N M R}\left(151 \mathrm{MHz}, \mathrm{CDCl}_{3}\right): \delta 170.8,156.1,155.7,82.0,80.5$, 80.0, 51.6, 44.8, 28.5, 28.4, 27.30, 25.9. ESI-MS: Calcd for $\mathrm{C}_{16} \mathrm{H}_{28} \mathrm{~N}_{2} \mathrm{O}_{6} \mathrm{Na}\left([\mathrm{M}+\mathrm{Na}]^{+}\right)$: 367.1845, Found: 367.1836.

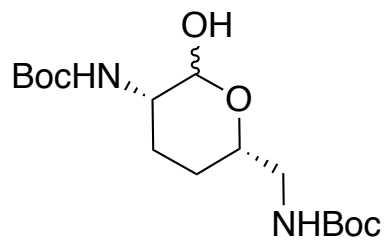

tert-Butyl (((2S,5S)-5-((tert-butoxycarbonyl)amino)-6-hydroxytetrahydro-2H-pyran-2-yl)methyl)carbamate $((2 \mathrm{~L}, 5 S)-16)$. Synthesized according to the general protocol reported in the literature. ${ }^{8}$ In a $25 \mathrm{~mL}$ roundbottom flask, tert-butyl (((2S,5S)-5-((tert-butoxycarbonyl)amino)-6oxotetrahydro-2H-pyran-2-yl)methyl)carbamate ((2L,5S)-15) (37.1 mg, 0.108 mmol, 1 equiv) was dissolved in $\mathrm{CH}_{2} \mathrm{Cl}_{2}(1.5 \mathrm{~mL})$. The mixture was cooled to $-78{ }^{\circ} \mathrm{C}$ and DIBAL $(1.0 \mathrm{M}$ in toluene, $0.162 \mathrm{~mL}, 0.162 \mathrm{mmol}, 1.5$ equiv) was added dropwise. The mixture was stirred at -78 ${ }^{\circ} \mathrm{C}$ for $2 \mathrm{~h}$, then $1.0 \mathrm{~mL} \mathrm{MeOH}$ was added at $-78{ }^{\circ} \mathrm{C}$ to quench the reaction. A solution of Rochelle's salt $(5 \mathrm{~mL})$ and $\mathrm{CH}_{2} \mathrm{Cl}_{2}(5 \mathrm{~mL})$ were added. The mixture was warmed to rt and layers were separated. The aq layer was extracted with $\mathrm{CH}_{2} \mathrm{Cl}_{2}(3 \times 10 \mathrm{~mL})$. The combined organic extracts were washed with brine $(3 \times 15 \mathrm{~mL})$, dried $\left(\mathrm{Na}_{2} \mathrm{SO}_{4}\right)$, filtered, and concentrated by rotary evaporation. Purification by silica flash chromatography $\left(3 \% \mathrm{MeOH}\right.$ in $\left.\mathrm{CH}_{2} \mathrm{Cl}_{2}\right)$ yielded the lactol (2L,5S)-16 as a colorless oil (36.9 $\mathrm{mg}, 99 \%$ yield).

TLC: $\mathrm{R}_{\mathrm{f}} 0.30\left(3 \% \mathrm{MeOH}\right.$ in $\left.\mathrm{CH}_{2} \mathrm{Cl}_{2}, \mathrm{KMnO}_{4}\right)$. Optical Rotation: $[\alpha]_{\mathrm{D}}{ }^{20}: 29.6^{\circ}$ (c 1.00, $\left.\mathrm{CHCl}_{3}\right)$. IR (ZnSe, film): 3345 (br), 2977 (m), 2932 (m), 1687 (s), 1508 (s), 1366 (m), 1248 (m), 1165 (s), $1068(\mathrm{~m}), 1025(\mathrm{~m}), 919(\mathrm{~m}) \mathrm{cm}^{-1}$. ${ }^{1} \mathbf{H}-\mathbf{N M R}\left(600 \mathrm{MHz}, \mathrm{CDCl}_{3}\right)$ : Major anomer $(\alpha): 5.19-$ $5.07(\mathrm{~m}, 1 \mathrm{H}), 5.02(\mathrm{~d}, J=3.5 \mathrm{~Hz}, 1 \mathrm{H}), 5.02-4.97(\mathrm{~m}, 1 \mathrm{H}), 4.06(\mathrm{tt}, J=8.1,3.4 \mathrm{~Hz}, 1 \mathrm{H}), 3.70$

${ }^{8}$ Jin, T.; Kim, J.-S.; Mu, Y.; Park, S.-H.; Jin, X.; Kang, J.-C.; Oh, C.-Y.; Ham, W.-H. Tetrahedron 2014, 70 (15), 2570 . 
$(\mathrm{d}, J=8.9 \mathrm{~Hz}, 1 \mathrm{H}), 3.65-3.53(\mathrm{~m}, 1 \mathrm{H}), 3.50-3.36(\mathrm{~m}, 1 \mathrm{H}), 3.11-2.99(\mathrm{~m}, 1 \mathrm{H}), 2.14-2.01$ $(\mathrm{m}, 1 \mathrm{H}), 1.71-1.63(\mathrm{~m}, 1 \mathrm{H}), 1.57-1.34(\mathrm{~m}, 18 \mathrm{H}), 1.39-1.25(\mathrm{~m}, 2 \mathrm{H})$. Minor anomer $(\beta)$ : $5.02-4.97(\mathrm{~m}, 1 \mathrm{H}), 4.97-4.91(\mathrm{~m}, 1 \mathrm{H}), 4.76(\mathrm{~d}, J=6.4 \mathrm{~Hz}, 1 \mathrm{H}), 4.56-4.38(\mathrm{br}, 1 \mathrm{H}), 3.81(\mathrm{~d}$, $J=7.6 \mathrm{~Hz}, 1 \mathrm{H}), 3.65-3.53(\mathrm{~m}, 1 \mathrm{H}), 3.33-3.24(\mathrm{~m}, 1 \mathrm{H}), 3.11-2.99(\mathrm{~m}, 1 \mathrm{H}), 1.96(\mathrm{dq}, J=$ 14.3, 3.7 Hz, 1H), 1.74 (q, $J=12.6 \mathrm{~Hz}, 1 \mathrm{H}), 1.57-1.34(\mathrm{~m}, 18 \mathrm{H}), 1.39-1.25(\mathrm{~m}, 2 \mathrm{H}) .{ }^{13} \mathrm{C}$ NMR (151 MHz, $\left.\mathbf{C D C l}_{3}\right)$ : Major anomer $(\alpha): \delta 156.2,155.4,93.6,79.6,67.8,47.2,44.9,28.5$, 23.2, 22.1. Minor anomer $(\beta): \delta$ 156.2, 155.4, 95.7, 80.5, 80.3, 75.8, 48.9, 45.1, 28.5, 27.0, 22.8 .

ESI-MS: Calcd for $\mathrm{C}_{16} \mathrm{H}_{31} \mathrm{~N}_{2} \mathrm{O}_{6}\left([\mathrm{M}+\mathrm{H}]^{+}\right): 347.2182$, Found: 347.2184 .

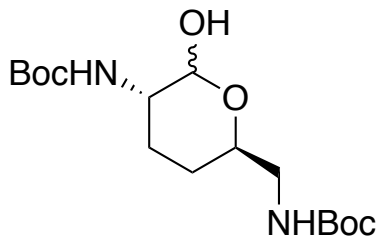

tert-Butyl (((2R,5S)-5-((tert-butoxycarbonyl)amino)-6-hydroxytetrahydro-2H-pyran-2-yl)methyl)carbamate $((\mathbf{2} \mathrm{L}, \mathbf{5} R)-16)$. Synthesized by the procedure above from $(\mathbf{2} \mathrm{L}, \mathbf{5} R)-15$ as a colorless oil (38.5 mg, 99\% yield).

TLC: $\mathrm{R}_{\mathrm{f}} 0.30\left(3 \% \mathrm{MeOH}\right.$ in $\left.\mathrm{CH}_{2} \mathrm{Cl}_{2}, \mathrm{KMnO}_{4}\right)$. Optical Rotation: $[\alpha]_{\mathrm{D}}{ }^{20}:-34.1^{\circ}(c 1.00$, $\mathrm{CHCl}_{3}$ ). IR (ZnSe, film): 3355 (br), 2978 (m), 2933 (m), 1690 (s), 1508 (s), 1366 (m), 1249 (m), $1165(\mathrm{~s}), 999(\mathrm{~m}) \mathrm{cm}^{-1}$. ${ }^{1}$ H-NMR $\left(600 \mathrm{MHz}, \mathrm{CDCl}_{3}\right)$ : Major anomer $(\alpha): \delta 5.13(\mathrm{~d}, J=3.2 \mathrm{~Hz}$, $1 \mathrm{H}), 5.07-4.94(\mathrm{~m}, 1 \mathrm{H}), 4.81(\mathrm{~d}, J=9.4 \mathrm{~Hz}, 1 \mathrm{H}), 3.96(\mathrm{ddt}, J=13.0,6.7,2.8 \mathrm{~Hz}, 1 \mathrm{H}), 3.76-$ $3.60(\mathrm{~m}, 2 \mathrm{H}), 3.28$ (tdd, $J=19.1,15.4,13.7,8.7 \mathrm{~Hz}, 2 \mathrm{H}), 3.04(\mathrm{qd}, J=13.7,12.1,5.4 \mathrm{~Hz}, 2 \mathrm{H})$, $1.85-1.76(\mathrm{~m}, 1 \mathrm{H}), 1.70(\mathrm{qd}, J=12.7,4.0 \mathrm{~Hz}, 1 \mathrm{H}), 1.66-1.57(\mathrm{~m}, 2 \mathrm{H}), 1.45-1.40(\mathrm{~m}, 18 \mathrm{H})$. Minor anomer $(\beta): \delta 5.36(\mathrm{~d}, J=19.8 \mathrm{~Hz}, 1 \mathrm{H}), 5.17(\mathrm{t}, J=6.0 \mathrm{~Hz}, 1 \mathrm{H}), 4.68(\mathrm{~d}, J=6.5 \mathrm{~Hz}, 1 \mathrm{H})$, $4.38(\mathrm{t}, J=6.8 \mathrm{~Hz}, 1 \mathrm{H}), 3.58-3.49(\mathrm{~m}, 1 \mathrm{H}), 3.41(\mathrm{ddd}, J=14.0,7.7,2.8 \mathrm{~Hz}, 1 \mathrm{H}), 3.34-3.21$ $(\mathrm{m}, 2 \mathrm{H}), 1.85-1.76(\mathrm{~m}, 1 \mathrm{H}), 1.70(\mathrm{qd}, J=12.7,4.0 \mathrm{~Hz}, 1 \mathrm{H}), 1.66-1.57(\mathrm{~m}, 2 \mathrm{H}), 1.45-1.40$ $(\mathrm{m}, 18 \mathrm{H}) .{ }^{13} \mathbf{C}-\mathbf{N M R}\left(151 \mathrm{MHz}, \mathrm{CDCl}_{3}\right)$ : Major anomer $(\alpha)$ : $\delta 156.3,155.4,91.7,79.6,67.6$, 49.3, 44.5, 28.5, 28.4, 27.6, 24.1. Minor anomer $(\beta): \delta 156.2,155.4,100.2,81.0,75.2,53.2$, 44.7, 28.6, 28.5, 27.8., 24.1. ESI-MS: Calcd for $\mathrm{C}_{16} \mathrm{H}_{30} \mathrm{~N}_{2} \mathrm{O}_{6} \mathrm{Na}\left([\mathrm{M}+\mathrm{Na}]^{+}\right): 369.2002$, Found: 369.1994 .<smiles></smiles>

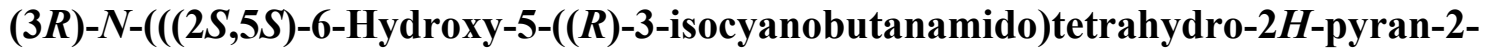
yl)methyl)-3-isocyanobutanamide $((2 \mathrm{~L}, 5 S)-1$; SF2768). In a $10 \mathrm{~mL}$ roundbottom flask, tertbutyl (((2S,5S)-5-((tert-butoxycarbonyl)amino)-6-hydroxytetrahydro-2H-pyran-2-yl)methyl)carbamate ((2L,5S)-16) (36.9 mg, $0.107 \mathrm{mmol}, 1.0$ equiv) was dissolved in $\mathrm{CH}_{2} \mathrm{Cl}_{2}(1.0 \mathrm{~mL})$. The mixture was cooled to $0{ }^{\circ} \mathrm{C}$, the TFA $(0.25 \mathrm{~mL})$ was added. The mixture was warmed to rt over $1 \mathrm{~h}$, then the solvent was removed by rotary evaporation to afford the crude $(3 S, 6 S)$-3-amino-6(aminomethyl)tetrahydro-2H-pyran-2-ol TFA salt ((2L,5S)-6) (40.0 mg, quantitative yield), which was carried forward without further purification. 
In a $10 \mathrm{~mL}$ roundbottom flask, 2,5-dioxopyrrolidin-1-yl $(R)$-3-isocyanobutanoate 5 (56.1 $\mathrm{mg}$, $0.267 \mathrm{mmol}, 2.5$ equiv) was dissolved in $\mathrm{CH}_{2} \mathrm{Cl}_{2}(1.5 \mathrm{~mL})$. The mixture was cooled to $0{ }^{\circ} \mathrm{C}$ followed by addition of diisopropylethylamine $(0.093 \mathrm{~mL}, 0.534 \mathrm{mmol}, 5.0$ equiv). In a separate flask, (3S,6S)-3-amino-6-(aminomethyl)tetrahydro-2H-pyran-2-ol TFA salt ((2L,5S)-6) (40.0 mg, $0.107 \mathrm{mmol}, 1$ equiv) was dissolved in dry $\mathrm{MeOH}(0.5 \mathrm{~mL})$, then added dropwise to the solution of 5 at $0{ }^{\circ} \mathrm{C}$ using a syringe pump over $20 \mathrm{~min}$. The mixture was warmed to $\mathrm{rt}$ and stirred for $1.5 \mathrm{~h}$, then concentrated by rotary evaporation. Purification by silica flash chromatography ( $2 \%$ $\mathrm{MeOH}$ in $\left.\mathrm{CH}_{2} \mathrm{Cl}_{2}\right)$ yielded SF2768 $((\mathbf{2} \mathrm{L}, 5 \mathrm{~S})-1)$ as a colorless oil $(18.0 \mathrm{mg}, 50 \%$ yield over two steps, $\alpha / \beta$ ratio $=1.2: 1$ in DMSO- $d_{6}$ or $1: 1.2$ in $\left.\mathrm{D}_{2} \mathrm{O}\right)$, having spectra data consistent with those reported in the literature (see SECTION F below). 9,10

TLC: $\mathrm{R}_{\mathrm{f}} 0.50\left(10 \% \mathrm{MeOH}\right.$ in $\left.\mathrm{CH}_{2} \mathrm{Cl}_{2}, \mathrm{KMnO}_{4}\right)$. Optical Rotation: $[\alpha]_{\mathrm{D}}{ }^{20}: 21.5^{\circ}(c 1.00$, MeOH). IR (ZnSe, film): 3306 (br), 2986 (w), 2941 (m), 2142 (s), 1647 (s), 1542 (s), 1202 (m), $1132(\mathrm{w}), 1036(\mathrm{w}), 912(\mathrm{w}) \mathrm{cm}^{-1}$.

${ }^{1}$ H NMR $\left(600 \mathrm{MHz}, \mathrm{DMSO}-d_{6}\right)$ : Major anomer $(\alpha)$ : $\delta 8.04(\mathrm{dt}, J=8.2,5.6 \mathrm{~Hz}, 2 \mathrm{H}), 6.40(\mathrm{~d}, J=$ $4.3 \mathrm{~Hz}, 1 \mathrm{H}), 4.79(\mathrm{~d}, J=4.2 \mathrm{~Hz}, 1 \mathrm{H}), 4.08$ (tq, $J=6.8 \mathrm{~Hz}, 2 \mathrm{H}), 3.88$ (dtd, $J=11.8,6.2,2.4 \mathrm{~Hz}$, $1 \mathrm{H}), 3.68-3.59(\mathrm{~m}, 1 \mathrm{H}), 3.20-3.07(\mathrm{~m}, 2 \mathrm{H}), 2.61(\mathrm{dd}, J=14.4,8.6 \mathrm{~Hz}, 1 \mathrm{H}), 2.47-2.39(\mathrm{~m}$, $3 \mathrm{H}), 1.92(\mathrm{ddt}, J=13.4,3.9 \mathrm{~Hz}, 1 \mathrm{H}), 1.56-1.44(\mathrm{~m}, 2 \mathrm{H}), 1.43-1.35(\mathrm{~m}, 1 \mathrm{H}), 1.32-1.28(\mathrm{~m}$, $6 \mathrm{H})$. Minor anomer $(\beta): \delta 8.08(\mathrm{t}, J=5.7 \mathrm{~Hz}, 1 \mathrm{H}), 7.74(\mathrm{~d}, J=8.2 \mathrm{~Hz}, 1 \mathrm{H}), 6.63(\mathrm{~d}, J=5.7 \mathrm{~Hz}$, $1 \mathrm{H}), 4.69(\mathrm{dd}, J=5.7,1.9 \mathrm{~Hz}, 1 \mathrm{H}), 4.08(\mathrm{tq}, J=6.8 \mathrm{~Hz}, 2 \mathrm{H}), 3.82(\mathrm{dq}, J=8.3,3.0 \mathrm{~Hz}, 1 \mathrm{H}), 3.52$ $(\mathrm{dtd}, J=10.5,5.5,2.9 \mathrm{~Hz}, 1 \mathrm{H}), 3.30(\mathrm{dt}, J=13.3,5.9 \mathrm{~Hz}, 1 \mathrm{H}), 3.20-3.07(\mathrm{~m}, 1 \mathrm{H}), 2.66(\mathrm{dd}, J=$ 14.4, 8.7 Hz, 1H), $2.47-2.39(\mathrm{~m}, 3 \mathrm{H}), 1.72(\mathrm{ddt}, J=13.6,3.8 \mathrm{~Hz}, 1 \mathrm{H}), 1.64-1.57(\mathrm{~m}, 1 \mathrm{H})$, $1.43-1.35(\mathrm{~m}, 1 \mathrm{H}), 1.36-1.32(\mathrm{~m}, 1 \mathrm{H}), 1.32-1.28(\mathrm{~m}, 6 \mathrm{H}) .{ }^{13} \mathbf{C}-\mathbf{N M R}(151 \mathrm{MHz}, \mathrm{DMSO}-$ $\left.d_{6}\right):{ }^{11}$ Major anomer $(\alpha): \delta 168.2,168.0,154.9(2 \mathrm{C}), 91.8,66.1,47.3,47.1,46.4,43.3,42.2,42.0$, 23.0, 21.7, 21.1, 21.0. Minor anomer $(\beta)$ : $\delta$ 168.4, 168.3, 154.9 (2C), 94.3, 73.7, 47.3, 47.2, 47.1, 43.1, 42.2, 42.0, 26.6, 22.4, 21.1, 21.0.
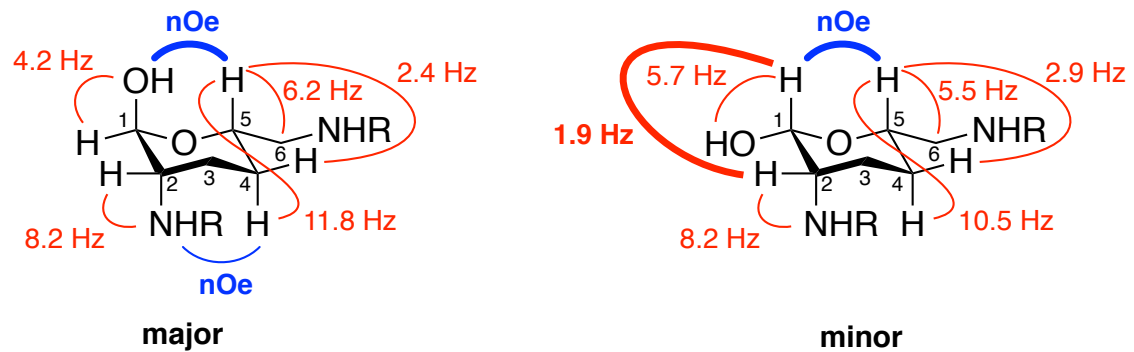

The major $(\alpha)$ and minor $(\beta)$ anomers were assigned based on analyses of coupling constants and nOe interactions above (DMSO- $\left.d_{6}\right)$. $\mathrm{H} 1-\mathrm{H} 2$ coupling in the major anomer was not observed.

${ }^{1}$ H-NMR (600 MHz, D $\left.2 \mathrm{O}\right)$ : Major anomer $(\beta): \delta 4.90(\mathrm{~d}, J=1.8 \mathrm{~Hz}, 1 \mathrm{H}), 4.17-4.10(\mathrm{~m}, 2 \mathrm{H})$, $4.01(\mathrm{~d}, J=1.9 \mathrm{~Hz}, 1 \mathrm{H}), 3.79-3.69(\mathrm{~m}, 1 \mathrm{H}), 3.39(\mathrm{dd}, J=14.1,4.1 \mathrm{~Hz}, 1 \mathrm{H}), 3.35-3.27(\mathrm{~m}$, $1 \mathrm{H}), 2.69(\mathrm{dd}, J=14.3,10.0 \mathrm{~Hz}, 1 \mathrm{H}), 2.60-2.56(\mathrm{~m}, 2 \mathrm{H}), 2.58-2.54(\mathrm{~m}, 1 \mathrm{H}), 1.92-1.85(\mathrm{~m}$,

\footnotetext{
${ }^{9}$ Tabata, Y.; Hatsu, M.; Amano, S.; Shimizu, A.; Imai, S. Meiji Seika Kenkyu Nenpo 1995, 34, 1-9.

${ }^{10} \mathrm{He}$ and coworkers also reported the spectra of SF2768, see: Wang, L.; Zhu, M.; Zhang, Q.; Zhang, X.; Yang, P.; Liu, Z.; Deng, Y.; Zhu, Y.; Huang, X.; Han, L.; Li, S.; He, J. ACS Chem. Biol. 2017, 12 (12), 3067.

11 The C-N coupling constants of the isonitrile carbons (R-NC) and vicinal carbons ( $\left.\mathrm{R}_{2} \mathrm{CH}-\mathrm{NC}\right)$ could not be determined due to overlapping signals from the two anomers and/or non-equivalent isonitrile side chains.
} 
$1 \mathrm{H}), 1.85-1.77(\mathrm{~m}, 1 \mathrm{H}), 1.51-1.44(\mathrm{~m}, 2 \mathrm{H}), 1.43-1.37(\mathrm{~m}, 6 \mathrm{H})$. Minor anomer $(\alpha): \delta 5.03(\mathrm{~s}$, $1 \mathrm{H}), 4.18-4.10(\mathrm{~m}, 2 \mathrm{H}), 4.08(\mathrm{dd}, J=9.2,5.2 \mathrm{~Hz}, 1 \mathrm{H}), 3.84-3.78(\mathrm{~m}, 1 \mathrm{H}), 3.33-3.27(\mathrm{~m}$, 2H), $2.65-2.60(\mathrm{~m}, 1 \mathrm{H}), 2.59-2.57(\mathrm{~m}, 2 \mathrm{H}), 2.57-2.54(\mathrm{~m}, 1 \mathrm{H}), 2.08-1.99(\mathrm{~m}, 1 \mathrm{H}), 1.71$ $(\mathrm{dq}, J=14.3,3.6 \mathrm{~Hz}, 1 \mathrm{H}), 1.59-1.52(\mathrm{~m}, 2 \mathrm{H}), 1.42-1.37(\mathrm{~m}, 6 \mathrm{H}) .{ }^{13} \mathrm{C}-\mathbf{N M R}(151 \mathrm{MHz}$, $\left.\mathrm{D}_{2} \mathrm{O}\right):{ }^{11}$ Major anomer $(\beta): \delta 173.4,173.0,152.0(2 \mathrm{C}), 95.1,75.9,49.0,48.8\left(2 \mathrm{C} ; \mathrm{C}^{2}, \mathrm{C}^{3^{\prime} / 3^{\prime \prime}}\right)$, 44.2, 43.4, 43.4, 27.4, 23.0, 21.4, 21.3. Minor anomer $(\alpha)$ : $\delta 173.1,172.6,152.0,151.9,92.9$, $68.3,48.8,48.8(2 \mathrm{C}), 48.0,44.2,43.4,43.2,23.4,22.3,21.3(2 \mathrm{C})$.

ESI-MS: Calcd for $\mathrm{C}_{16} \mathrm{H}_{24} \mathrm{~N}_{4} \mathrm{O}_{4} \mathrm{Na}\left([\mathrm{M}+\mathrm{Na}]^{+}\right)$: 359.1695 , Found: 359.1706 .<smiles>CC(N)CC(=O)N[C@H]1CC[C@@H](CNC(=O)C[C@H](C)[N])O[C@H]1O</smiles>

$(3 R)-N-(((2 R, 5 S)-6-H y d r o x y-5-((R)-3-i s o c y a n o b u t a n a m i d o) t e t r a h y d r o-2 H-p y r a n-2-y l)-$ methyl)-3-isocyanobutanamide $((2 \mathrm{~L}, 5 R)-1 ; 5-e p i-S F 2768)$. Synthesized by the procedure above from $(\mathbf{2} \mathbf{L}, \mathbf{5 R})-16$ as a white solid $(10.1 \mathrm{mg}, 27 \%$ yield over two steps, $\alpha / \beta$ ratio $=1.3: 1$ in DMSO- $d_{6}$ or $1: 1.1$ in $\left.\mathrm{D}_{2} \mathrm{O}\right)$.

TLC: $\mathrm{R}_{\mathrm{f}} 0.50\left(10 \% \mathrm{MeOH}\right.$ in $\left.\mathrm{CH}_{2} \mathrm{Cl}_{2}, \mathrm{KMnO}_{4}\right)$. mp: $170-171{ }^{\circ} \mathrm{C}$. Optical Rotation: $[\alpha]_{\mathrm{D}}{ }^{20}$ : $-38.8^{\circ}$ (c 0.50, MeOH). IR (ZnSe, film): 3295 (br), 2985 (w), 2941 (m), 2142 (s), 1641 (s), $1558(\mathrm{~s}), 1451(\mathrm{w}), 1380(\mathrm{w}), 1209(\mathrm{w}), 1063(\mathrm{~m}) \mathrm{cm}^{-1}$.

${ }^{1}$ H-NMR (600 MHz, DMSO- $\left.d_{6}\right)$ : Major anomer $(\alpha): \delta 8.10(\mathrm{t}, J=6.0 \mathrm{~Hz}, 1 \mathrm{H}), 7.84(\mathrm{~d}, J=8.4$ $\mathrm{Hz}, 1 \mathrm{H}), 6.49$ (dd, $J=4.4,1.2 \mathrm{~Hz}, 1 \mathrm{H}), 4.92(\mathrm{dd}, J=3.8 \mathrm{~Hz}, 1 \mathrm{H}), 4.13-3.97(\mathrm{~m}, 2 \mathrm{H}), 3.86$ (dtd, $J=11.5,5.5,2.2 \mathrm{~Hz}, 1 \mathrm{H}), 3.73(\mathrm{ddt}, J=12.9,8.6,3.8 \mathrm{~Hz}, 1 \mathrm{H}), 3.23-3.08(\mathrm{~m}, 1 \mathrm{H}), 3.04(\mathrm{dt}, J=$ $13.4,5.7 \mathrm{~Hz}, 1 \mathrm{H}), 2.59-2.50(\mathrm{~m}, 2 \mathrm{H}), 2.46-2.27(\mathrm{~m}, 2 \mathrm{H}), 1.70(\mathrm{qd}, J=12.7,4.0 \mathrm{~Hz}, 1 \mathrm{H}), 1.50$ $(\mathrm{dq}, J=11.7,3.7 \mathrm{~Hz}, 1 \mathrm{H}), 1.34(\mathrm{dd}, J=13.2,4.0 \mathrm{~Hz}, 1 \mathrm{H}), 1.32-1.26(\mathrm{~m}, 6 \mathrm{H}), 1.26-1.16(\mathrm{~m}$, $1 \mathrm{H})$. Minor anomer $(\beta): \delta 8.19(\mathrm{t}, J=5.9 \mathrm{~Hz}, 1 \mathrm{H}), 7.99(\mathrm{~d}, J=8.4 \mathrm{~Hz}, 1 \mathrm{H}), 6.52(\mathrm{~d}, J=6.6 \mathrm{~Hz}$, $1 \mathrm{H}), 4.37$ (dd, $J=8.2,6.6 \mathrm{~Hz}, 1 \mathrm{H}), 4.13-3.97(\mathrm{~m}, 2 \mathrm{H}), 3.49-3.38(\mathrm{~m}, 2 \mathrm{H}), 3.23-3.08(\mathrm{~m}$, $2 \mathrm{H}), 2.59-2.50(\mathrm{~m}, 2 \mathrm{H}), 2.46-2.27(\mathrm{~m}, 2 \mathrm{H}), 1.84(\mathrm{dq}, J=12.7,3.7 \mathrm{~Hz}, 1 \mathrm{H}), 1.66-1.55(\mathrm{~m}$, $3 \mathrm{H}), 1.32-1.26(\mathrm{~m}, 6 \mathrm{H}) .{ }^{13} \mathrm{C}-\mathrm{NMR}\left(151 \mathrm{MHz}, \mathrm{DMSO}-d_{6}\right):{ }^{11}$ Major anomer $(\alpha): \delta 168.3$, $167.8,154.9$ (2C), 90.0, 65.6, 48.4, 47.2, 47.1, 43.0, 42.2, 42.0, 28.0, 23.1, 21.0. Minor anomer (ß): $\delta 168.4,167.7,154.8(2 \mathrm{C}), 97.1,73.7,50.8,47.2,47.1,43.0,42.4,42.1,28.8,27.8,21.0$.
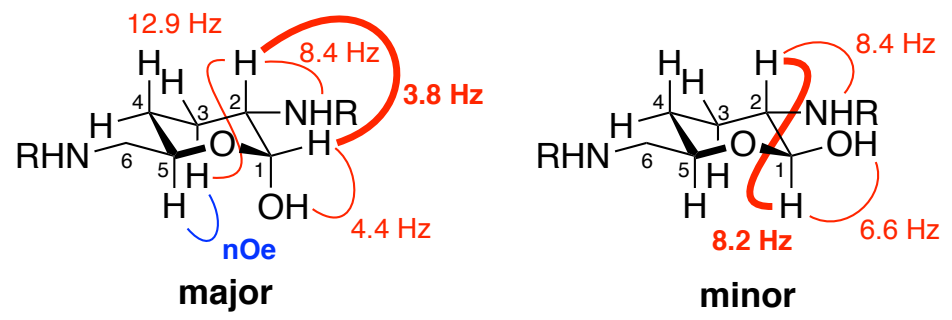

The major $(\alpha)$ and minor $(\beta)$ anomers were assigned based on analyses of coupling constants and nOe interactions above (DMSO- $d_{6}$ ). $\mathrm{H} 1-\mathrm{H} 5$ nOe interaction in the minor anomer could not be observed due to overlap of the $\mathrm{H} 2$ and $\mathrm{H} 5$ proton signals. 
${ }^{1}$ H NMR (600 MHz, D $\left.2 \mathrm{O}\right)$ Major anomer $(\beta): \delta 4.59(\mathrm{~d}, J=8.4 \mathrm{~Hz}, 1 \mathrm{H}), 4.18-4.08(\mathrm{~m}, 2 \mathrm{H})$, $3.71(\mathrm{dtd}, J=11.3,5.6,1.9 \mathrm{~Hz}, 1 \mathrm{H}), 3.58(\mathrm{ddd}, J=12.6,8.4,4.6 \mathrm{~Hz}, 1 \mathrm{H}), 3.33(\mathrm{~d}, J=5.6 \mathrm{~Hz}$, $2 \mathrm{H}), 2.61-2.51(\mathrm{~m}, 4 \mathrm{H}), 2.05-1.98(\mathrm{~m}, 1 \mathrm{H}), 1.59-1.47(\mathrm{~m}, 2 \mathrm{H}), 1.46-1.39(\mathrm{~m}, 1 \mathrm{H}), 1.41-$ $1.34(\mathrm{~m}, 6 \mathrm{H})$. Minor anomer $(\alpha): \delta 5.16(\mathrm{~d}, J=3.3 \mathrm{~Hz}, 1 \mathrm{H}), 4.18-4.06(\mathrm{~m}, 2 \mathrm{H}), 4.04-3.97(\mathrm{~m}$, 1H), 3.89 (ddd, $J=10.5,6.7,3.3 \mathrm{~Hz}, 1 \mathrm{H}), 3.31-3.28(\mathrm{~m}, 2 \mathrm{H}), 2.64-2.49(\mathrm{~m}, 4 \mathrm{H}), 1.79-1.73$ $(\mathrm{m}, 2 \mathrm{H}), 1.80-1.67(\mathrm{~m}, 2 \mathrm{H}), 1.41-1.35(\mathrm{~m}, 6 \mathrm{H}) .{ }^{13} \mathbf{C}$ NMR $\left(151 \mathrm{MHz}, \mathrm{D}_{2} \mathrm{O}\right):{ }^{11}$ Major anomer $(\beta): \delta 172.0,172.0,151.0(2 \mathrm{C}), 151.0,96.6,74.6,51.2,47.8(2 \mathrm{C}), 43.1,42.6,42.4,27.9,27.0$, 20.3 (2C). Minor anomer $(\alpha)$ : $\delta 171.7,171.5,151.0,150.9,90.2,66.8,48.7,47.8(2 \mathrm{C}), 43.0$, $42.5,42.2,26.9,22.2,20.3(2 \mathrm{C})$.

ESI-MS: Calcd for $\mathrm{C}_{16} \mathrm{H}_{24} \mathrm{~N}_{4} \mathrm{O}_{4} \mathrm{Na}\left([\mathrm{M}+\mathrm{Na}]^{+}\right)$: 359.1695 , Found: 359.1685 . 


\section{F. COMPARISON OF NMR DATA FOR SYNTHETIC SF2768, 5-epi-SF2768, AND LITERATURE REPORTS}

Comparison of NMR data for (2L,5S)-1 and natural SF2768 in DMSO-d 6

Table S1. Comparison of ${ }^{1} \mathrm{H}-\mathrm{NMR}$ data for synthetic (2L,5S)-1 and natural SF2768 in DMSO- $d_{6} .{ }^{9}$

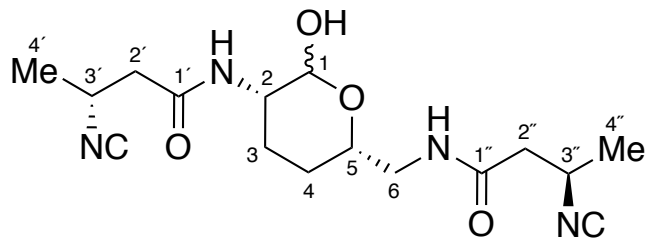

\begin{tabular}{|c|c|c|c|c|c|c|}
\hline \multirow[b]{2}{*}{$\mathbf{H}$} & \multicolumn{3}{|l|}{ major $(\alpha)$ anomer } & \multicolumn{3}{|c|}{ minor $(\beta)$ anomer } \\
\hline & synthetic & lit. & $\Delta$ & synthetic & lit. & $\Delta$ \\
\hline 1 & $4.79(\mathrm{~d}, J=4.2 \mathrm{~Hz}, 1 \mathrm{H})$ & 4.80 & -0.01 & $4.69(\mathrm{dd}, J=5.7,1.9 \mathrm{~Hz}, 1 \mathrm{H})$ & 4.69 & 0.00 \\
\hline 1-OH & $6.40(\mathrm{~d}, J=4.3 \mathrm{~Hz}, 1 \mathrm{H})$ & 6.35 & +0.05 & $6.63(\mathrm{~d}, J=5.7 \mathrm{~Hz}, 1 \mathrm{H})$ & 6.62 & +0.01 \\
\hline 2 & $3.68-3.59(\mathrm{~m}, 1 \mathrm{H})$ & 3.63 & & $3.82(\mathrm{dq}, J=8.3,3.0 \mathrm{~Hz}, 1 \mathrm{H})$ & 3.83 & -0.01 \\
\hline 2-NH & $8.04(\mathrm{dt}, J=8.2,5.6 \mathrm{~Hz}, 1 \mathrm{H})$ & 8.00 & +0.04 & $7.74(\mathrm{~d}, J=8.2 \mathrm{~Hz}, 1 \mathrm{H})$ & 7.71 & +0.03 \\
\hline \multirow[t]{2}{*}{3} & 1.92 (ddt, $J=13.4,3.9 \mathrm{~Hz}, 1 \mathrm{H})$ & 1.90 & +0.02 & 1.72 (ddt, $J=13.6,3.8 \mathrm{~Hz}, 1 \mathrm{H})$ & $1.2 \sim 1.7$ & \\
\hline & $1.56-1.44(\mathrm{~m}, 1 \mathrm{H})$ & 1.66 & & $1.64-1.57(\mathrm{~m}, 1 \mathrm{H})$ & & \\
\hline \multirow[t]{2}{*}{4} & $1.56-1.44(\mathrm{~m}, 1 \mathrm{H})$ & 1.50 & & $1.43-1.35(\mathrm{~m}, 1 \mathrm{H})$ & $1.2 \sim 1.7$ & \\
\hline & $1.43-1.35(\mathrm{~m}, 1 \mathrm{H})$ & & & $1.36-1.32(\mathrm{~m}, 1 \mathrm{H})$ & & \\
\hline 5 & $3.88(\mathrm{dtd}, J=11.8,6.2,2.4 \mathrm{~Hz}, 1 \mathrm{H})$ & 3.84 & +0.04 & $3.52(\mathrm{dtd}, J=10.5,5.5,2.9 \mathrm{~Hz}, 1 \mathrm{H})$ & 3.53 & -0.01 \\
\hline \multirow[t]{2}{*}{6} & $3.20-3.07(\mathrm{~m}, 2 \mathrm{H})$ & 3.15 & & $3.30^{*}(\mathrm{dt}, J=13.3,5.9 \mathrm{~Hz}, 1 \mathrm{H})$ & 3.15 & \\
\hline & & & & $3.20-3.07(\mathrm{~m}, 1 \mathrm{H})$ & & \\
\hline 6-NH & $8.04(\mathrm{dt}, J=7.0,4.4 \mathrm{~Hz}, 1 \mathrm{H})$ & 8.00 & +0.04 & $8.08(\mathrm{t}, \mathrm{J}=5.7 \mathrm{~Hz}, 1 \mathrm{H})$ & 8.00 & +0.08 \\
\hline \multirow[t]{2}{*}{$2^{\prime}$} & $2.47-2.39(\mathrm{~m}, 1 \mathrm{H})$ & 2.46 & & $2.47-2.39(\mathrm{~m}, 1 \mathrm{H})$ & 2.46 & \\
\hline & $2.61(\mathrm{dd}, J=14.4,8.6 \mathrm{~Hz}, 1 \mathrm{H})$ & 2.61 & 0.00 & $2.61(\mathrm{dd}, J=14.4,8.6 \mathrm{~Hz}, 1 \mathrm{H})$ & 2.61 & 0.00 \\
\hline $3^{\prime}$ & $4.08(\mathrm{tq}, J=6.8 \mathrm{~Hz}, 1 \mathrm{H})$ & 4.10 & -0.02 & $4.08(\mathrm{tq}, J=6.8 \mathrm{~Hz}, 1 \mathrm{H})$ & 4.10 & -0.02 \\
\hline $4^{\prime}$ & $1.32-1.28(\mathrm{~m}, 3 \mathrm{H})$ & 1.28 & & $1.32-1.28(\mathrm{~m}, 3 \mathrm{H})$ & 1.28 & \\
\hline $2 "$ & $2.47-2.39(\mathrm{~m}, 2 \mathrm{H})$ & 2.46 & & $2.47-2.39(\mathrm{~m}, 2 \mathrm{H})$ & 2.46 & \\
\hline $3 "$ & $4.08(\mathrm{tq}, J=6.8 \mathrm{~Hz}, 1 \mathrm{H})$ & 4.10 & -0.02 & $4.08(\mathrm{tq}, J=6.8 \mathrm{~Hz}, 1 \mathrm{H})$ & 4.10 & -0.02 \\
\hline $4 "$ & $1.32-1.28(\mathrm{~m}, 3 \mathrm{H})$ & 1.28 & & $1.32-1.28(\mathrm{~m}, 3 \mathrm{H})$ & 1.28 & \\
\hline
\end{tabular}

DMSO-d 6 referenced to 2.50 ppm for synthetic sample. *See COSY spectrum in SECTION L below for assignment of this additional peak, which appears not to have been assigned in the literature. 
Table S2. Comparison of ${ }^{13} \mathrm{C}-\mathrm{NMR}$ data for synthetic (2L,5S)-1 and natural SF2768 in DMSO- $d_{6} .{ }^{9}$

\begin{tabular}{|l|c|c|r|r|r|r|}
\hline & \multicolumn{2}{|c|}{ major $(\boldsymbol{\alpha})$ anomer } & \multicolumn{3}{c|}{ minor $(\boldsymbol{\beta})$ anomer } \\
\hline $\mathbf{C}$ & synthetic & literature & \multicolumn{1}{|c|}{$\boldsymbol{\Delta}$} & synthetic & literature & \multicolumn{1}{c|}{$\boldsymbol{\Delta}$} \\
\hline $\mathbf{1}$ & 91.8 & 92.3 & -0.5 & 94.3 & 94.7 & -0.4 \\
\hline $\mathbf{2}$ & 46.4 & 46.8 & -0.4 & 47.2 & 47.6 & -0.4 \\
\hline $\mathbf{3}$ & 21.7 & 22.1 & -0.4 & 22.4 & 22.8 & -0.4 \\
\hline $\mathbf{4}$ & 23.0 & 23.4 & -0.4 & 26.6 & 26.9 & -0.3 \\
\hline $\mathbf{5}$ & 66.1 & 66.6 & -0.5 & 73.7 & 74.1 & -0.4 \\
\hline $\mathbf{6}$ & 43.3 & 43.8 & -0.5 & 43.1 & 43.5 & -0.4 \\
\hline $\mathbf{1}^{\prime}$ & 168.2 & 168.6 & -0.4 & 168.2 & 168.6 & -0.4 \\
\hline $\mathbf{2}^{\prime}$ & 42.0 & 42.5 & -0.5 & 42.0 & 42.5 & -0.5 \\
\hline $\mathbf{3}^{\prime}$ & 47.1 & 47.4 & -0.3 & 47.1 & 47.4 & -0.3 \\
\hline $\mathbf{3}^{\prime}-\mathrm{NC}$ & 154.9 & 155.4 & -0.5 & 154.9 & 155.4 & -0.5 \\
\hline $\mathbf{4}^{\prime}$ & 21.1 & 21.5 & -0.4 & 21.1 & 21.5 & -0.4 \\
\hline $\mathbf{1}^{\prime \prime}$ & 168.0 & 168.4 & -0.4 & 168.0 & 168.4 & -0.4 \\
\hline $\mathbf{2}^{\prime \prime}$ & 42.2 & 42.7 & -0.5 & 42.2 & 42.7 & -0.5 \\
\hline $\mathbf{3}^{\prime \prime}$ & 47.3 & 47.4 & -0.1 & 47.3 & 47.4 & -0.1 \\
\hline $\mathbf{3}^{\prime \prime-N C}$ & 154.9 & 155.4 & -0.5 & 154.9 & 155.4 & -0.5 \\
\hline $\mathbf{4}^{\prime \prime}$ & 21.0 & 21.5 & -0.5 & 21.0 & 21.5 & -0.5 \\
\hline
\end{tabular}

DMSO- $d_{6}$ referenced to $2.50 \mathrm{ppm}$ for synthetic sample.

Comparison of NMR data for (2L,5S)-1 (SF2768) and (2L,5R)-1 (5-epi-SF2768) in DMSO-d

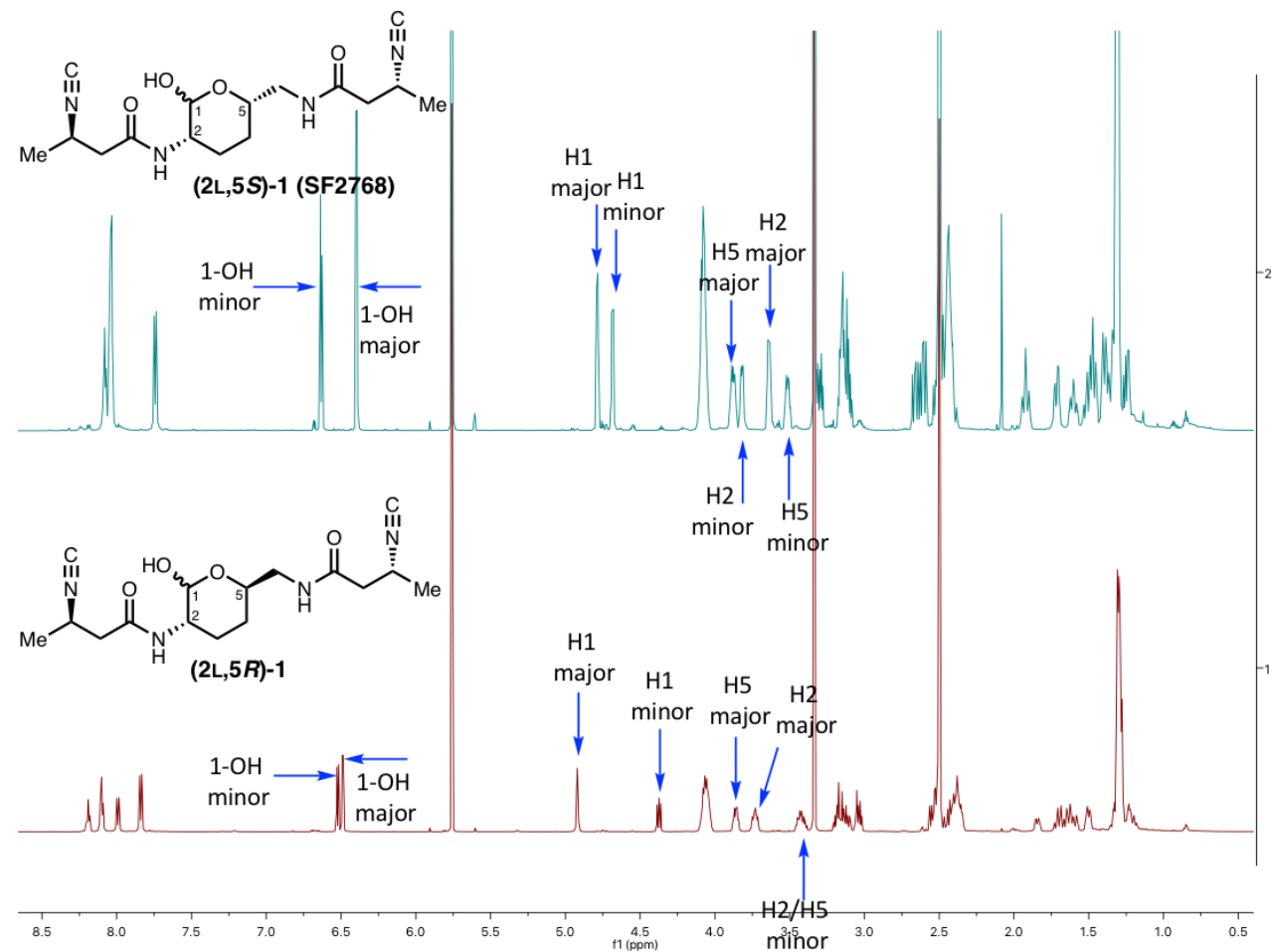

Figure S5. ${ }^{1} \mathrm{H}-\mathrm{NMR}$ spectra of synthetic (2L,5S)-1 (SF2768) and (2L,5R)-1 (5-epi-SF2768) in DMSO- $d_{6}$. 
Table S3. Diagnostic ${ }^{1} \mathrm{H}-\mathrm{NMR}$ resonances for (2L,5S)-1 (SF2768) and (2L,5R)-1 (5-epi-SF2768) in DMSO-d ${ }_{6}$.

\begin{tabular}{|c|c|c|c|c|}
\hline & \multicolumn{2}{|c|}{ major $(\alpha)$ anomer } & \multicolumn{2}{|c|}{ minor $(\beta)$ anomer } \\
\hline $\mathbf{H}$ & $(2 L, 5 S)-1$ & $(2 \mathrm{~L}, 5 R)-1$ & $(2 L, 5 S)-1$ & $(2 \mathrm{~L}, 5 R)-1$ \\
\hline 1 & $\begin{array}{l}4.79 \\
(\mathrm{~d}, \mathrm{~J}=4.2 \mathrm{~Hz}, 1 \mathrm{H})\end{array}$ & $\begin{array}{l}4.92 \\
(\mathrm{dd}, \mathrm{J}=3.8 \mathrm{~Hz}, 1 \mathrm{H})\end{array}$ & $\begin{array}{l}4.69 \\
(\mathrm{dd}, \mathrm{J}=5.7,1.9 \mathrm{~Hz} \text {, } \\
1 \mathrm{H})\end{array}$ & $\begin{array}{l}4.37 \\
(\mathrm{dd}, \mathrm{J}=8.2,6.6 \mathrm{~Hz}, \\
1 \mathrm{H})\end{array}$ \\
\hline $1-\mathrm{OH}$ & $\begin{array}{l}6.40 \\
(\mathrm{~d}, \mathrm{~J}=4.3 \mathrm{~Hz}, 1 \mathrm{H})\end{array}$ & $\begin{array}{l}6.49 \\
(\mathrm{dd}, \mathrm{J}=4.4,1.2 \mathrm{~Hz} \\
1 \mathrm{H})\end{array}$ & $\begin{array}{l}6.63 \\
(d, J=5.7 \mathrm{~Hz}, 1 \mathrm{H})\end{array}$ & $\begin{array}{l}6.52 \\
(d, J=6.6 \mathrm{~Hz}, 1 \mathrm{H})\end{array}$ \\
\hline 2 & $\begin{array}{l}3.68-3.59 \\
(m, 1 H)\end{array}$ & $\begin{array}{l}3.73 \\
(\mathrm{ddt}, \mathrm{J}=12.9,8.6 \\
3.8 \mathrm{~Hz}, 1 \mathrm{H})\end{array}$ & $\begin{array}{l}3.82 \\
(\mathrm{dq}, J=8.3,3.0 \mathrm{~Hz}, \\
1 \mathrm{H})\end{array}$ & $\begin{array}{l}3.49-3.38 \\
(\mathrm{~m}, 1 \mathrm{H})\end{array}$ \\
\hline 5 & $\begin{array}{l}3.88 \\
(\mathrm{dtd}, \mathrm{J}=11.8,6.2 \\
2.4 \mathrm{~Hz}, 1 \mathrm{H})\end{array}$ & $\begin{array}{l}3.86 \\
(\mathrm{dtd}, \mathrm{J}=11.5,5.5 \\
2.2 \mathrm{~Hz}, 1 \mathrm{H})\end{array}$ & $\begin{array}{l}3.52 \\
(\mathrm{dtd}, \mathrm{J}=10.5,5.5 \\
2.9 \mathrm{~Hz}, 1 \mathrm{H})\end{array}$ & $\begin{array}{l}3.49-3.38 \\
(\mathrm{~m}, 1 \mathrm{H})\end{array}$ \\
\hline
\end{tabular}

$D M S O-d_{6}$ referenced to $2.50 \mathrm{ppm}$.

Table S4. Diagnostic ${ }^{13} \mathrm{C}-\mathrm{NMR}$ resonances for (2L,5S)-1 (SF2768) and (2L,5R)-1 (5-epi-SF2768) in DMSO-d $_{6}$

\begin{tabular}{|l|c|c|c|c|}
\hline & \multicolumn{2}{|c|}{ major $(\boldsymbol{\alpha})$ anomer } & \multicolumn{2}{c|}{ minor $(\boldsymbol{\beta})$ anomer } \\
\hline $\mathbf{C}$ & $\mathbf{( 2 L , 5 S ) - 1}$ & $\mathbf{( 2 L , 5 R ) - 1}$ & $\mathbf{( 2 L , 5 S ) - 1}$ & $\mathbf{( 2 L , 5 R ) - 1}$ \\
\hline $\mathbf{1}$ & 91.8 & 90.0 & 94.3 & 97.1 \\
\hline $\mathbf{2}$ & 46.4 & 48.4 & 47.2 & 50.8 \\
\hline $\mathbf{5}$ & 66.1 & 65.6 & 73.7 & 73.7 \\
\hline
\end{tabular}

DMSO-d ${ }_{6}$ referenced to $39.52 \mathrm{ppm}$. 


\section{Comparison of NMR data for (2L,5S)-1 and natural SF2768 in $\mathrm{D}_{2} \mathrm{O}$}

We also obtained ${ }^{1} \mathrm{H}-\mathrm{NMR}$ and ${ }^{13} \mathrm{C}-\mathrm{NMR}$ spectra of natural SF2768 in $\mathrm{D}_{2} \mathrm{O}$ from Professor Kenji Ueda, Nihon University. ${ }^{12}$ Stacked spectra of natural SF2768, synthetic (2L,5S)-1 (syndiastereomer), and its C5-epimer (2L,5R)-1 (anti-diastereomer) are shown below and further confirm the structural assignment of SF2768 as (2L,5S)-1 (syn-diastereomer).

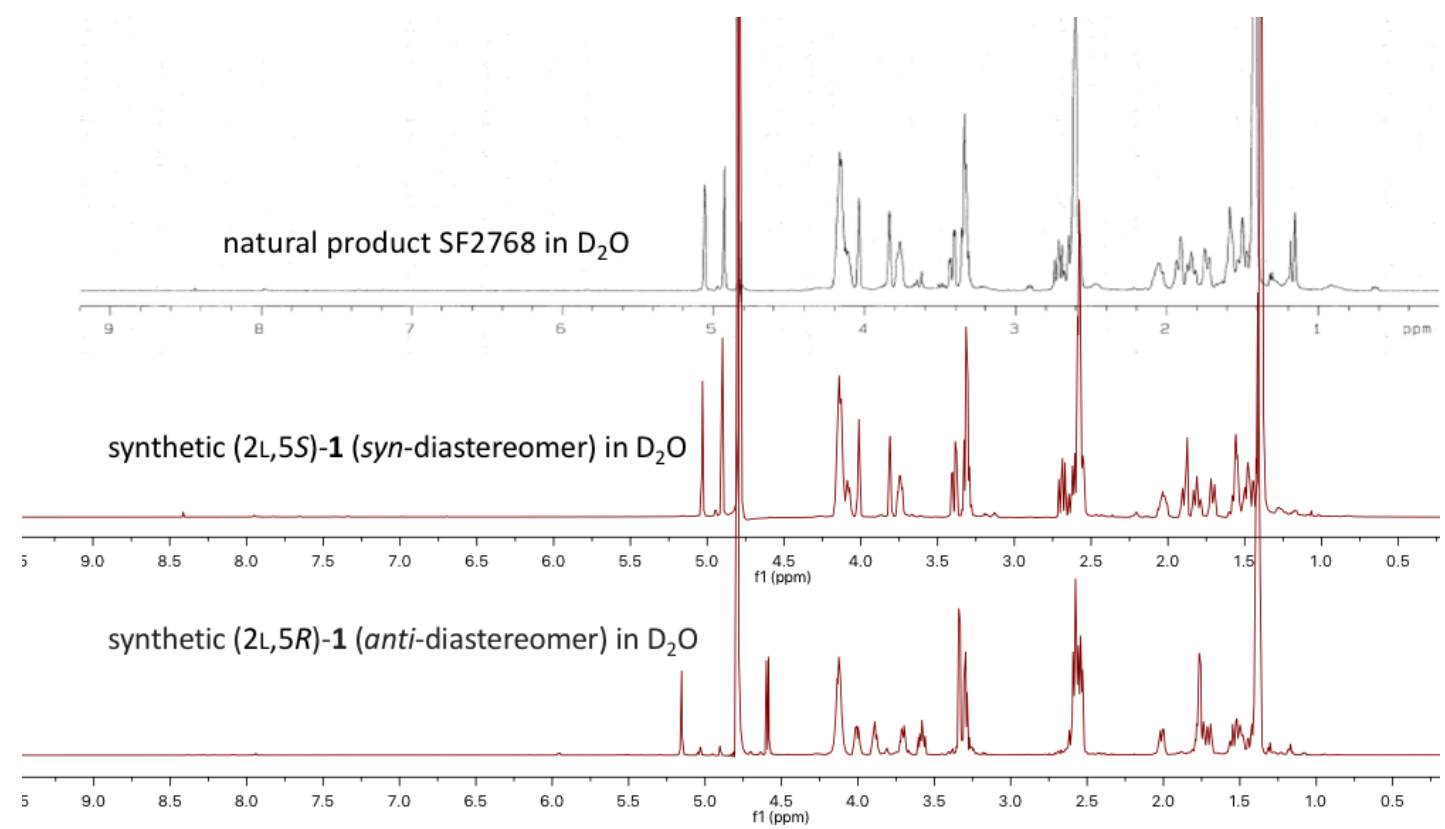

Figure S6. ${ }^{1} \mathrm{H}-\mathrm{NMR}$ spectra of natural SF2768, and synthetic (2L,5S)-1 and (2L,5R)-1 in $\mathrm{D}_{2} \mathrm{O}$.

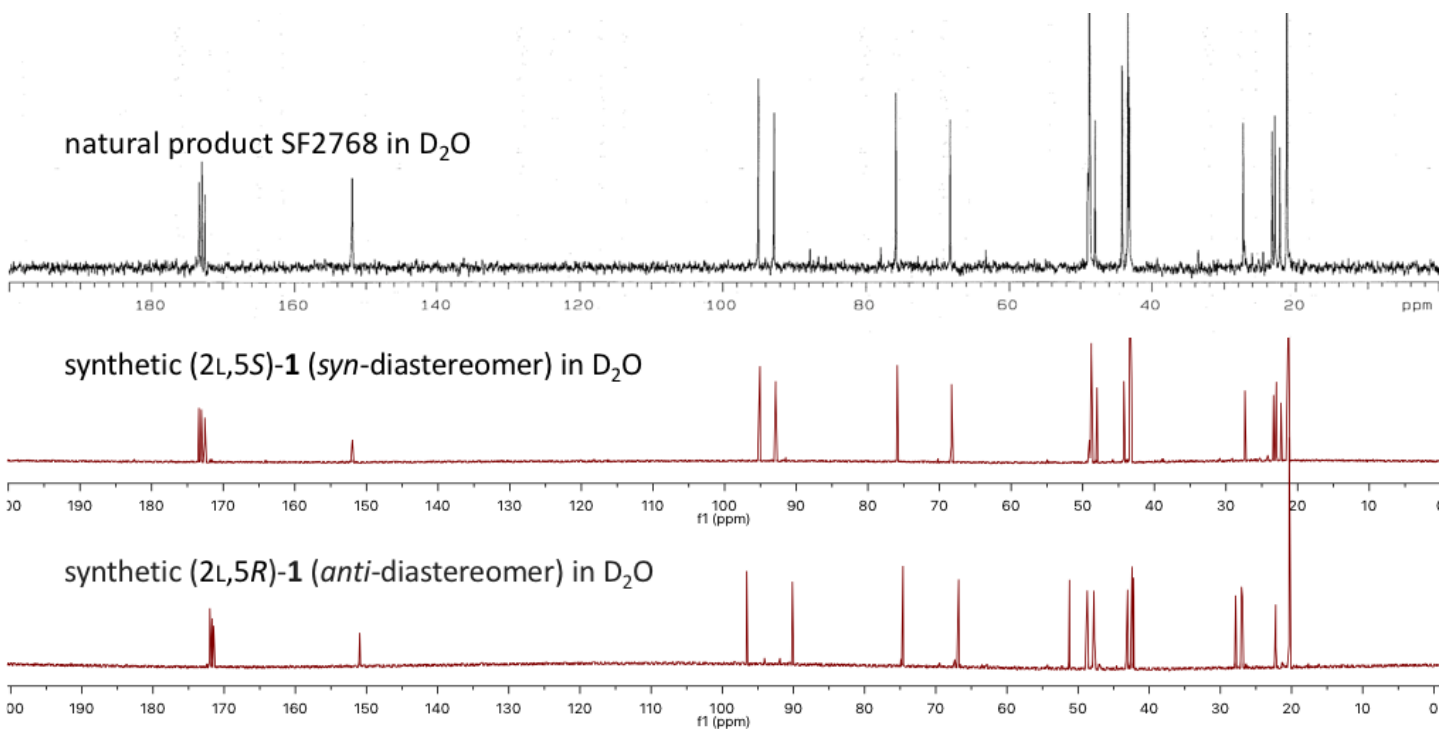

Figure S7. ${ }^{1} \mathrm{C}-\mathrm{NMR}$ spectra of natural SF2768, and synthetic (2L,5S)-1 and (2L,5R)-1 in $\mathrm{D}_{2} \mathrm{O}$.

${ }^{12}$ Amano, S.-i.; Sakurai, T.; Endo, K.; Takano, H.; Beppu, T.; Furihata, K.; Sakuda, S.; Ueda, K. “A cryptic antibiotic triggered by monensin." J. Antibiot. 2011, 64, 703; Prof. Ueda has informed us that the NMR spectra reported in the Supplementary Information of this reference were recorded in $\mathrm{D}_{2} \mathrm{O}$. 
Table S5. Comparison of ${ }^{1} \mathrm{H}-\mathrm{NMR}$ data for synthetic (2L,5S)-1 and natural SF2768 in $\mathrm{D}_{2} \mathrm{O}^{12}$

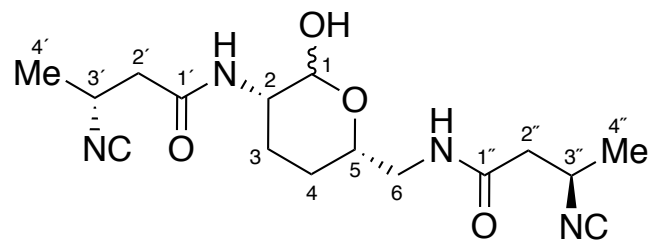

\begin{tabular}{|c|c|c|c|c|c|c|}
\hline & \multicolumn{3}{|c|}{ major $(\beta)$ anomer } & \multicolumn{3}{|c|}{ minor $(\alpha)$ anomer } \\
\hline $\mathbf{H}$ & synthetic & lit. & $\Delta$ & synthetic & lit. & $\Delta$ \\
\hline 1 & $4.90(\mathrm{~d}, J=1.8 \mathrm{~Hz}, 1 \mathrm{H})$ & 4.93 & -0.03 & $5.03(\mathrm{~s}, 1 \mathrm{H})$ & 5.06 & -0.03 \\
\hline $1-\mathrm{OH}$ & - & - & & - & - & \\
\hline 2 & $4.01(\mathrm{~d}, J=1.9 \mathrm{~Hz}, 1 \mathrm{H})$ & 4.04 & -0.03 & $3.84-3.78(\mathrm{~m}, 1 \mathrm{H})$ & 3.84 & \\
\hline 2-NH & - & - & & - & - & \\
\hline \multirow[t]{2}{*}{3} & $1.92-1.85(\mathrm{~m}, 1 \mathrm{H})$ & 1.92 & & $2.08-1.99(\mathrm{~m}, 1 \mathrm{H})$ & 2.06 & \\
\hline & $1.85-1.77(\mathrm{~m}, 1 \mathrm{H})$ & 1.84 & & $1.71(\mathrm{dq}, J=14.3,3.6 \mathrm{~Hz}, 1 \mathrm{H})$ & 1.74 & -0.03 \\
\hline \multirow[t]{2}{*}{4} & $1.51-1.44(\mathrm{~m}, 1 \mathrm{H})$ & 1.51 & & $1.59-1.52(\mathrm{~m}, 1 \mathrm{H})$ & 1.59 & \\
\hline & $1.51-1.44(\mathrm{~m}, 1 \mathrm{H})$ & 1.47 & & $1.59-1.52(\mathrm{~m}, 1 \mathrm{H})$ & 1.59 & \\
\hline 5 & $3.79-3.69(\mathrm{~m}, 1 \mathrm{H})$ & 3.77 & & $4.08(\mathrm{dd}, J=9.2,5.2 \mathrm{~Hz}, 1 \mathrm{H})$ & 4.11 & -0.03 \\
\hline \multirow[t]{2}{*}{6} & $3.39(\mathrm{dd}, J=14.1,4.1 \mathrm{~Hz}, 1 \mathrm{H})$ & 3.42 & -0.03 & $3.33-3.27(\mathrm{~m}, 1 \mathrm{H})$ & 3.34 & \\
\hline & $3.35-3.27(\mathrm{~m}, 1 \mathrm{H})$ & 3.34 & & $3.33-3.27(\mathrm{~m}, 1 \mathrm{H})$ & 3.34 & \\
\hline 6-NH & - & - & & - & - & \\
\hline \multirow[t]{2}{*}{$2^{\prime}$} & $2.69(\mathrm{dd}, J=14.3,10.0 \mathrm{~Hz}, 1 \mathrm{H})$ & 2.72 & -0.03 & $2.65-2.60(\mathrm{~m}, 1 \mathrm{H})$ & 2.66 & \\
\hline & $2.58-2.54(\mathrm{~m}, 1 \mathrm{H})$ & 2.60 & & $2.57-2.54(\mathrm{~m}, 1 \mathrm{H})$ & 2.60 & \\
\hline $3^{\prime}$ & $4.17-4.10(\mathrm{~m}, 1 \mathrm{H})$ & 4.17 & & $4.18-4.10(\mathrm{~m}, 1 \mathrm{H})$ & 4.17 & \\
\hline $4^{\prime}$ & $1.43-1.37(\mathrm{~m}, 3 \mathrm{H})$ & 1.43 & & $1.42-1.37(\mathrm{~m}, 3 \mathrm{H})$ & 1.43 & \\
\hline $2 "$ & $2.60-2.56(\mathrm{~m}, 2 \mathrm{H})$ & 2.61 & & $2.59-2.57(\mathrm{~m}, 2 \mathrm{H})$ & 2.61 & \\
\hline $3 "$ & $4.17-4.10(\mathrm{~m}, 1 \mathrm{H})$ & 4.17 & & $4.18-4.10(\mathrm{~m}, 1 \mathrm{H})$ & 4.17 & \\
\hline $4^{\prime \prime}$ & $1.43-1.37(\mathrm{~m}, 3 \mathrm{H})$ & 1.43 & & $1.42-1.37(\mathrm{~m}, 3 \mathrm{H})$ & 1.43 & \\
\hline
\end{tabular}

$D_{2} \mathrm{O}$ referenced to $4.79 \mathrm{ppm}$ for synthetic sample. 
Table S6. Comparisons of ${ }^{13} \mathrm{C}$-NMR data for synthetic (2L,5S)-1 and natural SF2768 in $\mathrm{D}_{2} \mathrm{O} . .^{12}$

\begin{tabular}{|c|c|c|c|c|c|c|}
\hline & \multicolumn{3}{|c|}{ Major $(\beta)$ anomer } & \multicolumn{3}{|c|}{ minor $(\alpha)$ anomer } \\
\hline C & synthetic & literature & $\Delta$ & synthetic & literature & $\Delta$ \\
\hline 1 & 95.1 & 95.0 & +0.1 & 92.9 & 92.9 & 0 \\
\hline 2 & 48.8 & 48.8 & 0 & 48.0 & 48.0 & 0 \\
\hline 3 & 27.4 & 27.3 & +0.1 & 22.3 & 22.3 & 0 \\
\hline 4 & 23.0 & 22.9 & +0.1 & 23.4 & 23.3 & +0.1 \\
\hline 5 & 75.9 & 75.9 & 0 & 68.3 & 68.3 & 0 \\
\hline 6 & 44.2 & 44.2 & 0 & 44.2 & 44.2 & 0 \\
\hline $1^{\prime}$ & 173.4 & 173.4 & 0 & 172.6 & 172.6 & 0 \\
\hline $2^{\prime}$ & 43.4 & 43.4 & 0 & 43.2 & 43.2 & 0 \\
\hline 3 & 49.0 & 49.0 & 0 & 48.8 & 48.8 & 0 \\
\hline $3^{\prime}-\mathrm{NC}$ & 152.0 & 151.9 & +0.1 & 152.0 & 151.9 & +0.1 \\
\hline 4 & 21.4 & 21.3 & +0.1 & 21.3 & 21.3 & 0 \\
\hline $1 "$ & 173.0 & 173.0 & 0 & 173.1 & 173.0 & +0.1 \\
\hline $2 "$ & 43.4 & 43.4 & 0 & 43.4 & 43.4 & 0 \\
\hline $3^{\prime \prime \prime}$ & 48.8 & 48.8 & 0 & 48.8 & 48.8 & 0 \\
\hline 3"-NC & 152.0 & 151.9 & +0.1 & 151.9 & 151.9 & 0 \\
\hline $4 "$ & 21.3 & 21.3 & 0 & 21.3 & 21.3 & 0 \\
\hline
\end{tabular}




\section{G. SYNTHESIS OF ACYCLIC DIISONITRILES 3 AND 4 AND COMPARISON OF NMR DATA}

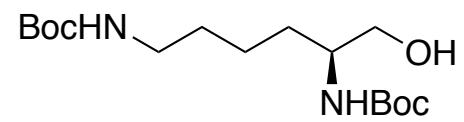

Di-tert-butyl (6-hydroxyhexane-1,5-diyl)(S)-dicarbamate (S5). Synthesized as previously described, with full characterization herein. ${ }^{13}$ In a $100-\mathrm{mL}$ roundbottom flask, $N^{2}, N^{6}$-bis $($ tertbutoxycarbonyl)-L-lysine (S4) $(6.00 \mathrm{~g}, 17.31 \mathrm{mmol}, 1.0$ equiv) was dissolved in THF (34 mL, $0.5 \mathrm{M})$ and cooled to $-10{ }^{\circ} \mathrm{C}$. $N, N$-diisopropylethylamine $(3.32 \mathrm{~mL}, 19.04 \mathrm{mmol}, 1.1$ equiv) was added followed by isobutyl chloroformate $(2.25 \mathrm{~mL}, 17.31 \mathrm{mmol}, 1.0$ equiv). The mixture was stirred at $-10^{\circ} \mathrm{C}$ for $40 \mathrm{~min}$, resulting in formation of a white solid. The mixture was filtered and the filter cake washed with dry THF $(3 \times 5 \mathrm{~mL})$. The combined filtrate was cooled to $-10{ }^{\circ} \mathrm{C}$, then $\mathrm{NaBH}_{4}(0.982 \mathrm{~g}, 25.96 \mathrm{mmol}, 1.5$ equiv $)$ was added in portions, followed by $\mathrm{H}_{2} \mathrm{O}(5.77 \mathrm{~mL}, 3.0 \mathrm{M})$. The mixture was warmed to $\mathrm{rt}$ and stirred for $1 \mathrm{~h} . \mathrm{H}_{2} \mathrm{O}(50 \mathrm{~mL})$ was added and the mixture was extracted with EtOAc $(3 \times 40 \mathrm{~mL})$. The combined organic extracts were washed with brine $(3 \times 100 \mathrm{~mL})$, dried $\left(\mathrm{Na}_{2} \mathrm{SO}_{4}\right)$, filtered, and concentrated by rotary evaporation. Purification by silica flash chromatography (50\% EtOAc in hexanes) yielded lysinol S5 as a colorless oil (4.33 g, 75\% yield).

TLC: $\mathrm{R}_{\mathrm{f}} 0.40\left(50 \%\right.$ EtOAc in hexanes, $\left.\mathrm{KMnO}_{4}\right)$. Optical Rotation: $[\alpha]_{D}^{17}:-8.7^{\circ}$ (c 1.00, $\mathrm{CHCl}_{3}$ ). IR (ZnSe, film): 3344 (m), 2977 (m), 2934 (m), 1691 (s), 1526 (s), 1366 (m), 1251 (m), $1173(\mathrm{~s}) \mathrm{cm}^{-1} .{ }^{1} \mathbf{H}-\mathbf{N M R}\left(600 \mathrm{MHz}, \mathrm{CDCl}_{3}\right): \delta 4.83(\mathrm{~d}, J=7.5 \mathrm{~Hz}, 1 \mathrm{H}), 4.63(\mathrm{t}, J=6.2 \mathrm{~Hz}, 1 \mathrm{H})$, $3.69-3.43(\mathrm{~m}, 3 \mathrm{H}), 3.24-2.97(\mathrm{~m}, 2 \mathrm{H}), 1.43(\mathrm{~s}, 24 \mathrm{H}) .{ }^{13} \mathbf{C}-\mathbf{N M R}\left(150 \mathrm{MHz}, \mathrm{CDCl}_{3}\right): \delta 156.7$, 156.5, 79.7, 79.4, 65.5, 52.6, 39.8, 30.8, 30.2, 28.6, 28.5, 22.8. ESI-MS: Calcd for $\mathrm{C}_{16} \mathrm{H}_{32} \mathrm{~N}_{2} \mathrm{O}_{5} \mathrm{Na}\left([\mathrm{M}+\mathrm{Na}]^{+}\right): 355.2202$, Found: 355.2209 .

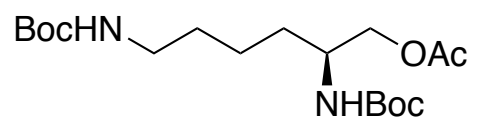

(S)-2,6-Bis((tert-butoxycarbonyl)amino)hexyl acetate (S6). Synthesized as previously described, with minor modifications. ${ }^{14}$ In a $100-\mathrm{mL}$ roundbottom flask, di-tert-butyl (6hydroxyhexane-1,5-diyl)(S)-dicarbamate (S5) (4.33 g, $13.02 \mathrm{mmol}, 1$ equiv) was dissolved in in $\mathrm{CH}_{2} \mathrm{Cl}_{2}(33 \mathrm{~mL}, 0.4 \mathrm{M})$. DMAP $\left(0.159 \mathrm{~g}, 1.30 \mathrm{mmol}, 0.1\right.$ equiv) and $\mathrm{Et}_{3} \mathrm{~N}(2.64 \mathrm{~g}, 26.04$ mmol, 2 equiv) were added, followed by dropwise addition of acetic anhydride (1.99 $\mathrm{g}, 19.52$ mmol, 1.5 equiv). The solution was stirred at $\mathrm{rt}$ for $1 \mathrm{~h}$, then $\mathrm{CH}_{2} \mathrm{Cl}_{2}(30 \mathrm{~mL})$ and satd aq $\mathrm{NaHCO}_{3}(80 \mathrm{~mL})$ were added. The organic layer was separated and the aqueous layer was extracted with EtOAc $(3 \times 30 \mathrm{~mL})$. The combined organic extracts were washed with brine $(3 \mathrm{x}$ $100 \mathrm{~mL})$, dried $\left(\mathrm{Na}_{2} \mathrm{SO}_{4}\right)$, filtered, and concentrated by rotary evaporation. Purification by silica flash chromatography $\left(5 \% \mathrm{MeOH}\right.$ in $\left.\mathrm{CH}_{2} \mathrm{Cl}_{2}\right)$ yielded acetate $\mathbf{S 6}$ as a colorless oil $(4.37 \mathrm{~g}, 90 \%$ yield).

TLC: $\mathrm{R}_{\mathrm{f}} 0.50\left(5 \% \mathrm{MeOH}\right.$ in $\left.\mathrm{CH}_{2} \mathrm{Cl}_{2}, \mathrm{KMnO}_{4}\right)$. Optical Rotation: $[\alpha]_{D}^{17}:-19.0^{\circ}(c 1.00$, $\mathrm{CHCl}_{3}$ ). IR (ZnSe, film): 3346 (m), 2977 (m), 2934 (m), 1690 (s), 1523 (s), 1366 (m), 1248 (m),

\footnotetext{
${ }^{13}$ Haghshenas, P.; Gravel, M. Org. Lett. 2016, 18 (18), 4518.

${ }^{14}$ Huang, X.; Rickman, B. H.; Borhan, B.; Berova, N.; Nakanishi, K. J. Am. Chem. Soc. 1998, 120 (24), 6185.
} 
$1171(\mathrm{~s}) \mathrm{cm}^{-1} .{ }^{1} \mathbf{H}-\mathbf{N M R}\left(500 \mathrm{MHz}, \mathrm{CDCl}_{3}\right): \delta 4.56(\mathrm{~d}, J=9.8 \mathrm{~Hz}, 1 \mathrm{H}), 4.10-3.97(\mathrm{~m}, 1 \mathrm{H})$, $3.87-3.75$ (rotamer, m, 1H), $3.15-3.04(\mathrm{~m}, 2 \mathrm{H}), 2.06(\mathrm{~s}, 3 \mathrm{H}), 1.73$ (rotamer, s, 3H), $1.60-$ $1.24(\mathrm{~m}, 24 \mathrm{H}) .{ }^{13} \mathbf{C}-\mathbf{N M R}\left(125 \mathrm{MHz}, \mathrm{CDCl}_{3}\right): \delta 171.1,156.2,155.7,79.6,79.2,66.5,49.6,40.4$, 31.7, 29.9, 28.6, 28.5, 23.1, 21.0. ESI-MS: Calcd for $\mathrm{C}_{18} \mathrm{H}_{34} \mathrm{~N}_{2} \mathrm{O}_{6} \mathrm{Na}\left([\mathrm{M}+\mathrm{Na}]^{+}\right): 397.2315$, Found: 397.2327.

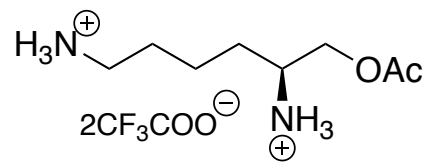

(S)-2,6-Diaminohexyl acetate, bis TFA salt (S7). Synthesized as previously described, with minor modifications. ${ }^{14}$ In a $25-\mathrm{mL}$ roundbottom flask, $(S)$-2,6-bis((tert-butoxycarbonyl)amino)hexyl acetate (S6) (4.35 g, $11.61 \mathrm{mmol}, 1.0$ equiv) was dissolved in $\mathrm{CH}_{2} \mathrm{Cl}_{2}(2.9 \mathrm{~mL}, 4.0 \mathrm{M})$. Trifluoroacetic acid (19.85 g, $174.1 \mathrm{mmol}, 15 \mathrm{equiv})$ was added dropwise. The mixture was stirred at $\mathrm{rt}$ for $16 \mathrm{~h}$, then concentrated by rotary evaporation to afford the crude diamine bis TFA salt S7 as a light yellow oil (quant. yield), which was carried forward without further purification.

Optical Rotation: $[\alpha]_{D}^{17}: 8.79^{\circ}(c 0.50, \mathrm{MeOH}) . \quad$ IR (ZnSe, film): $2952(\mathrm{w}), 1671(\mathrm{~s}), 1535(\mathrm{~m})$, 1431 (m), 1182 (s), 1131 (s), 1049 (w) cm ${ }^{-1}$. ${ }^{1}$ H-NMR (500 MHz, CD 3 OD): $\delta 4.34$ (dd, $J=$ $12.3,3.3 \mathrm{~Hz}, 1 \mathrm{H}), 4.15(\mathrm{dd}, J=12.3,6.7 \mathrm{~Hz}, 1 \mathrm{H}), 3.47(\mathrm{tt}, J=6.9,3.6 \mathrm{~Hz}, 1 \mathrm{H}), 2.95(\mathrm{t}, J=7.7$ $\mathrm{Hz}, 2 \mathrm{H}), 2.11(\mathrm{~s}, 3 \mathrm{H}), 1.82-1.62(\mathrm{~m}, 4 \mathrm{H}), 1.61-1.39(\mathrm{~m}, 2 \mathrm{H}) .{ }^{13} \mathbf{C}-\mathbf{N M R}\left(125 \mathrm{MHz}, \mathrm{CD}_{3} \mathrm{OD}\right)$ : $\delta 172.3,161.3\left(\mathrm{CH}_{3} \mathrm{COO}^{-}, \mathrm{q}, J=38.2 \mathrm{~Hz}\right), 117.1\left(\mathrm{CH}_{3} \mathrm{COO}^{-}, \mathrm{q}, J=288.4 \mathrm{~Hz}\right), 64.2,51.7,40.3$, $30.1,28.1,23.2,20.5$.

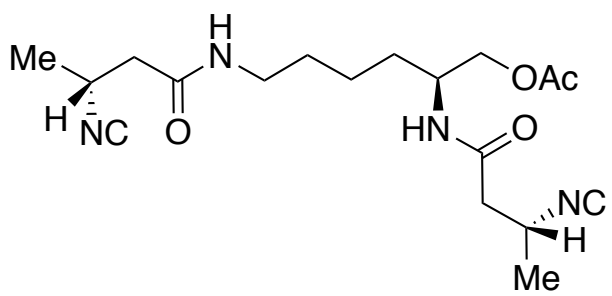

(S)-2,6-Bis((R)-3-isocyanobutanamido)hexyl acetate (4). In a 25-mL roundbottom flask, $(S)$ 2,6-diaminohexyl acetate TFA (S7) (0.656 g, $1.63 \mathrm{mmol}, 1$ equiv) was dissolved in THF (10 $\mathrm{mL}, 0.15 \mathrm{M})$. Distilled $N, N$-diisopropylethylamine $(1.26 \mathrm{~g}, 9.79 \mathrm{mmol}, 6$ equiv) was added. The mixture was cooled to $0^{\circ} \mathrm{C}$, then 2,5-dioxopyrrolidin-1-yl $(R)$-3-isocyanobutanoate $(\mathbf{5})(0.925 \mathrm{~g}$, $4.40 \mathrm{mmol}, 2.7$ equiv) was added in portions. The mixture was slowly warmed to $\mathrm{rt}$ and stirred for $6 \mathrm{~h}$. $\mathrm{CH}_{2} \mathrm{Cl}_{2}(30 \mathrm{~mL})$ and $\mathrm{H}_{2} \mathrm{O}(40 \mathrm{~mL})$ were added, the layers separated, and the aqueous layer extracted with $\mathrm{CH}_{2} \mathrm{Cl}_{2}(3 \times 30 \mathrm{~mL})$. The combined organic extracts were washed with $\mathrm{H}_{2} \mathrm{O}$ $(2 \times 100 \mathrm{~mL})$, dried $\left(\mathrm{Na}_{2} \mathrm{SO}_{4}\right)$, filtered, and concentrated by rotary evaporation. Purification by silica flash chromatography $\left(0.3 \% \mathrm{Et}_{3} \mathrm{~N}\right.$ in EtOAc) yielded the diisonitrile acetate 4 as a light yellow solid $\left(0.487 \mathrm{~g}, 82 \%\right.$ yield). ${ }^{1} \mathrm{H}-\mathrm{NMR}$ and ${ }^{13} \mathrm{C}-\mathrm{NMR}$ data were consistent with those reported for the natural product. ${ }^{15}$

\footnotetext{
${ }^{15}$ Harris, N. C.; Sato, M.; Herman, N. A.; Twigg, F.; Cai, W.; Liu, J.; Zhu, X.; Downey, J.; Khalaf, R.; Martin, J.; Koshino, H.; Zhang, W. Proc Natl Acad Sci USA 2017, 114 (27), 7025.
} 
TLC: $\mathrm{R}_{\mathrm{f}} 0.25\left(\right.$ EtOAc, $\left.\mathrm{KMnO}_{4}\right) . \quad \mathbf{m p}: 79-81^{\circ} \mathrm{C}$. Optical Rotation: $[\alpha]_{D}^{17}: 138.14^{\circ}(c 1.00$, $\left.\mathrm{CHCl}_{3}\right) . \quad$ IR (ZnSe, film): 3295 (m), 2941 (w), 2141 (m), 1738 (m), 1649 (s), 1548 (s), 1453 (w), $1235(\mathrm{~m}), 1045(\mathrm{w}) \mathrm{cm}^{-1}$. ${ }^{1}$ H-NMR $\left(600 \mathrm{MHz}, \mathrm{DMSO}-d_{6}\right): \delta 8.01(\mathrm{t}, J=5.6 \mathrm{~Hz}, 1 \mathrm{H}), 7.97(\mathrm{~d}, J$ $=8.4 \mathrm{~Hz}, 1 \mathrm{H}), 4.11-4.04(\mathrm{~m}, 2 \mathrm{H}), 4.00(\mathrm{dd}, J=10.7,4.7 \mathrm{~Hz}, 1 \mathrm{H}), 3.97-3.92(\mathrm{~m}, 1 \mathrm{H}), 3.84$ $(\mathrm{dd}, J=10.7,6.4 \mathrm{~Hz}, 1 \mathrm{H}), 3.08-2.96(\mathrm{~m}, 2 \mathrm{H}), 2.49-2.44(\mathrm{~m}, 2 \mathrm{H}), 2.44-2.33(\mathrm{~m}, 2 \mathrm{H}), 1.99$ $(\mathrm{s}, 3 \mathrm{H}), 1.56-1.13(\mathrm{~m}, 12 \mathrm{H}) .{ }^{13} \mathrm{C}-\mathbf{N M R}\left(150 \mathrm{MHz}, \mathrm{DMSO}-d_{6}\right): \delta 170.4,168.2,168.0,155.0(\mathrm{t}$, $J=4.4 \mathrm{~Hz}), 154.9$ (t, $J=4.8 \mathrm{~Hz}), 65.5,47.4,47.2(\mathrm{t}, J=5.4 \mathrm{~Hz})^{16}, 47.1(\mathrm{t}, J=5.6 \mathrm{~Hz}), 42.5$, 42.3, 38.4, 30.2, 28.9, 22.7, 21.1, 21.0, 20.8. ESI-MS: Calcd for $\mathrm{C}_{18} \mathrm{H}_{29} \mathrm{~N}_{4} \mathrm{O}_{4}\left([\mathrm{M}+\mathrm{H}]^{+}\right)$: 365.2189, Found: 365.2173.

${ }^{16}$ For discussion of spin coupling between ${ }^{14} \mathrm{~N}$ and ${ }^{13} \mathrm{C}$, see: Runge, W. and Firl, J., Z. Naturforsch., 1976, 31b, 1515-1518. 
Table S7. Comparisons of ${ }^{1} \mathrm{H}-\mathrm{NMR}$ and ${ }^{13} \mathrm{C}$-NMR data for synthetic and natural product diisonitrile acetate 4 in DMSO- $d_{6}$.

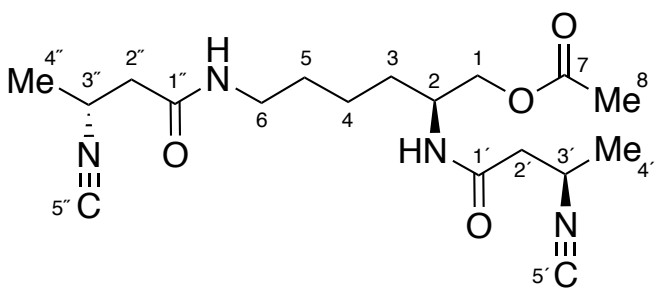

\begin{tabular}{|c|c|c|c|c|c|c|}
\hline & \multicolumn{3}{|c|}{${ }^{1} \mathrm{H}-\mathrm{NMR}$} & \multicolumn{3}{|c|}{${ }^{13} \mathrm{C}-\mathrm{NMR}$} \\
\hline $\mathrm{H} / \mathrm{C}$ & synthetic & literature $^{15}$ & $\Delta$ & syn. & lit. $^{15}$ & $\Delta$ \\
\hline 1 & $\begin{array}{l}3.84(d d, J=10.7,6.4 \\
H z, 1 H) \\
4.00(d d, J=10.7,4.7, H z \\
1 H)\end{array}$ & $\begin{array}{l}3.84(d d, J=10.9,6.5 \\
H z, 1 H) \\
3.99(d d, J=10.9,4.8 \mathrm{~Hz}, \\
1 \mathrm{H})\end{array}$ & $\begin{array}{r}0.00 \\
+0.01\end{array}$ & 65.5 & 65.4 & +0.1 \\
\hline 2 & 3.97-3.92 (m, 1H) & $3.94(\mathrm{~m}, 1 \mathrm{H})$ & & 47.4 & 47.3 & +0.1 \\
\hline $2-\mathrm{NH}$ & $7.97(\mathrm{~d}, J=8.4 \mathrm{~Hz}, 1 \mathrm{H})$ & $7.93(\mathrm{~d}, J=8.5 \mathrm{~Hz}, 1 \mathrm{H})$ & +0.04 & & & \\
\hline 3 & $1.56-1.13(\mathrm{~m}, 2 \mathrm{H})$ & $\begin{array}{l}1.47(\mathrm{~m}, 1 \mathrm{H}) \\
1.34(\mathrm{~m}, 1 \mathrm{H})\end{array}$ & & 30.2 & 30.2 & 0.0 \\
\hline 4 & $1.56-1.13(\mathrm{~m}, 2 \mathrm{H})$ & $\begin{array}{l}1.34(\mathrm{~m}, 1 \mathrm{H}) \\
1.26(\mathrm{~m}, 1 \mathrm{H})\end{array}$ & & 22.7 & 22.6 & +0.1 \\
\hline 5 & $1.56-1.13(\mathrm{~m}, 2 \mathrm{H})$ & $1.39(\mathrm{~m}, 2 \mathrm{H})$ & & 28.9 & 28.8 & +0.1 \\
\hline 6 & $3.08-2.96(\mathrm{~m}, 2 \mathrm{H})$ & $3.03(\mathrm{~m}, 2 \mathrm{H})$ & & 38.4 & 38.3 & +0.1 \\
\hline $6-\mathrm{NH}$ & $8.01(\mathrm{t}, J=5.6 \mathrm{~Hz}, 1 \mathrm{H})$ & $7.97(\mathrm{~m}, 1 \mathrm{H})$ & +0.04 & & & \\
\hline 7 & & & & 170.4 & 170.2 & +0.2 \\
\hline 8 & $1.99(\mathrm{~s}, 3 \mathrm{H})$ & $1.99(\mathrm{~s}, 3 \mathrm{H})$ & 0.00 & 20.8 & 20.6 & +0.2 \\
\hline $1^{\prime}$ & & & & 168.2 & 168.0 & +0.2 \\
\hline $2^{\prime}$ & $2.49-2.44(\mathrm{~m}, 2 \mathrm{H})$ & $\begin{array}{l}2.41(\mathrm{~m}, 1 \mathrm{H}) \\
2.47(\mathrm{~m}, 1 \mathrm{H})\end{array}$ & & 42.5 & 42.4 & +0.1 \\
\hline $3^{\prime}$ & $4.11-4.04(\mathrm{~m}, 1 \mathrm{H})$ & $4.07(\mathrm{~m}, 1 \mathrm{H})$ & & 47.2 & 47.1 & +0.1 \\
\hline $4^{\prime}$ & $1.33-1.28(\mathrm{~m}, 3 \mathrm{H})$ & $1.31(\mathrm{~m}, 3 \mathrm{H})$ & & 21.0 & 20.9 & +0.1 \\
\hline $5^{\prime}$ & & & & 155.0 & 155.0 & 0.0 \\
\hline $1^{\prime \prime}$ & & & & 168.0 & 167.8 & +0.2 \\
\hline $2 "$ & $2.44-2.33(\mathrm{~m}, 2 \mathrm{H})$ & $\begin{array}{l}2.37(\mathrm{~m}, 1 \mathrm{H}) \\
2.44(\mathrm{~m}, 1 \mathrm{H}) \\
\end{array}$ & & 42.3 & 42.3 & 0.0 \\
\hline $3 "$ & $4.11-4.04(\mathrm{~m}, 1 \mathrm{H})$ & $4.07(\mathrm{~m}, 1 \mathrm{H})$ & & 47.1 & 47.0 & +0.1 \\
\hline 4" & $1.33-1.28(\mathrm{~m}, 3 \mathrm{H})$ & $1.31(\mathrm{~m}, 3 \mathrm{H})$ & & 21.1 & 21.0 & +0.1 \\
\hline $5 "$ & & & & 154.9 & 154.9 & 0.0 \\
\hline
\end{tabular}

DMSO- $d_{6}$ referenced to $2.50 \mathrm{ppm}\left({ }^{1} \mathrm{H}\right)$ and $39.52 \mathrm{ppm}\left({ }^{13} \mathrm{C}\right)$ for synthetic sample. 


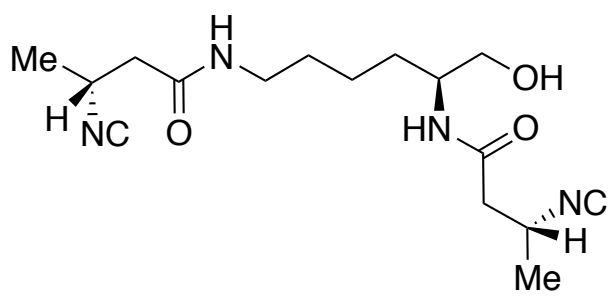

$\left(3 R, 3^{\prime} R\right)-N, N^{\prime}-((S)-6-H y d r o x y h e x a n e-1,5-d i y l) b i s(3-i s o c y a n o b u t a n a m i d e)(3) . \quad$ In a $10-\mathrm{mL}$ roundbottom flask, $(S)$-2,6-bis $((R)$-3-isocyanobutanamido)hexyl acetate (4) (0.141 g, 0.387 mmol, 1 equiv) and $\mathrm{K}_{2} \mathrm{CO}_{3}(0.535 \mathrm{~g}, 3.87 \mathrm{mmol}, 10$ equiv) were dissolved in $\mathrm{MeOH}(2.5 \mathrm{~mL})$ and $\mathrm{H}_{2} \mathrm{O}(0.25 \mathrm{~mL})\left(\mathrm{MeOH}: \mathrm{H}_{2} \mathrm{O}=10: 1 \mathrm{v} / \mathrm{v}\right)$. The mixture was stirred at $\mathrm{rt}$ for $3 \mathrm{~h}$, then filtered. The filter cake was washed with $\mathrm{MeOH}$ and the combined filtrate was concentrated by rotary evaporation. Purification by silica flash chromatography $(10 \% \mathrm{MeOH}$ in EtOAc) yielded diisonitrile alcohol 3 as a light yellow oil $\left(0.112 \mathrm{~g}, 90 \%\right.$ yield). The ${ }^{1} \mathrm{H}-\mathrm{NMR}$ and ${ }^{13} \mathrm{C}-\mathrm{NMR}$ data were consistent with those reported for the natural product. ${ }^{15,17}$

TLC: $\mathrm{R}_{\mathrm{f}} 0.25\left(10 \% \mathrm{MeOH}\right.$ in EtOAc, $\left.\mathrm{KMnO}_{4}\right)$. Optical Rotation: $[\alpha]_{D}^{17}: 182.0^{\circ}(c 1.00$, $\mathrm{CHCl}_{3}$ ). IR (ZnSe, film): 3298 (br), 2935 (m), 2141 (m), 1649 (s), 1557(m), 1456 (w), 1378 (m), $1260(\mathrm{w}), 1055(\mathrm{w}) \mathrm{cm}^{-1}$. ${ }^{1}$ H-NMR (600 MHz, DMSO- $\left.d_{6}\right): \delta 7.99(\mathrm{t}, J=5.6 \mathrm{~Hz}, 1 \mathrm{H}), 7.76$ $(\mathrm{d}, J=8.6 \mathrm{~Hz}, 1 \mathrm{H}), 4.65(\mathrm{t}, J=5.6 \mathrm{~Hz}, 1 \mathrm{H}), 4.11-3.99(\mathrm{~m}, 2 \mathrm{H}), 3.75-3.64(\mathrm{~m}, 1 \mathrm{H}), 3.34-$ $3.28(\mathrm{~m}, 1 \mathrm{H}), 3.27-3.19(\mathrm{~m}, 1 \mathrm{H}), 3.10-2.96(\mathrm{~m}, 2 \mathrm{H}), 2.48-2.41(\mathrm{~m}, 2 \mathrm{H}), 2.41-2.33(\mathrm{~m}$, 2H), $1.57-1.47(\mathrm{~m}, 1 \mathrm{H}), 1.44-1.11(\mathrm{~m}, 11 \mathrm{H}) .{ }^{13} \mathbf{C}-\mathbf{N M R}\left(150 \mathrm{MHz}, \mathrm{DMSO}-d_{6}\right): \delta 167.9(2 \mathrm{C})$, $154.9(2 \mathrm{C}, \mathrm{m}), 63.3,50.5,47.2(\mathrm{t}, J=5.5 \mathrm{~Hz}), 47.1(\mathrm{t}, J=5.5 \mathrm{~Hz}), 42.5,42.3,38.5,30.4,29.1$, 22.9, 21.0 (2C). ESI-MS: Calcd for $\mathrm{C}_{16} \mathrm{H}_{27} \mathrm{~N}_{4} \mathrm{O}_{3}\left([\mathrm{M}+\mathrm{H}]^{+}\right): 323.2083$, Found: 323.2074.

\footnotetext{
${ }^{17}$ He and coworkers also reported the spectra of 3 and 4, see: Wang, L.; Zhu, M.; Zhang, Q.; Zhang, X.; Yang, P.;
} Liu, Z.; Deng, Y.; Zhu, Y.; Huang, X.; Han, L.; Li, S.; He, J. ACS Chem. Biol. 2017, 12 (12), 3067. 
Table S8. Comparisons of ${ }^{1} \mathrm{H}-\mathrm{NMR}$ and ${ }^{13} \mathrm{C}$-NMR data for synthetic and natural product diisonitrile alcohol 3 in DMSO- $d_{6}$.

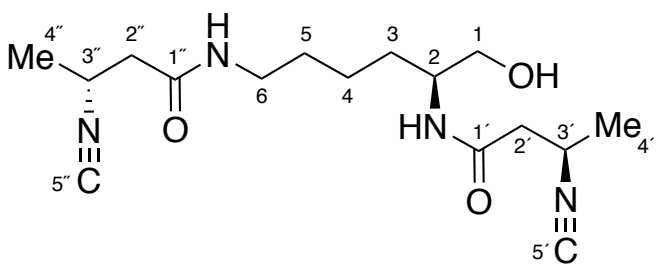

\begin{tabular}{|c|c|c|c|c|c|c|}
\hline & \multicolumn{3}{|c|}{${ }^{1} \mathrm{H}-\mathrm{NMR}$} & \multicolumn{3}{|c|}{${ }^{13} \mathrm{C}-\mathrm{NMR}$} \\
\hline $\mathrm{H} / \mathrm{C}$ & synthetic & literature $^{15}$ & $\Delta$ & syn. & lit. $^{15}$ & $\Delta$ \\
\hline 1 & $\begin{array}{l}3.27-3.19(\mathrm{~m}, 1 \mathrm{H}) \\
3.34-3.28(\mathrm{~m}, 1 \mathrm{H})\end{array}$ & $\begin{array}{l}3.24(\mathrm{~m}, 1 \mathrm{H}) \\
3.32(\mathrm{~m}, 1 \mathrm{H})\end{array}$ & & 63.3 & 63.3 & 0.0 \\
\hline $1-\mathrm{OH}$ & $4.65(\mathrm{t}, J=5.6 \mathrm{~Hz}, 1 \mathrm{H})$ & $4.65(\mathrm{~m}, 1 \mathrm{H})$ & 0.00 & & & \\
\hline 2 & 3.75-3.64 $(\mathrm{m}, 1 \mathrm{H})$ & $3.70(\mathrm{~m}, 1 \mathrm{H})$ & & 50.5 & 50.5 & 0.0 \\
\hline $2-\mathrm{NH}$ & $7.76(\mathrm{~d}, J=8.6 \mathrm{~Hz}, 1 \mathrm{H})$ & $7.76(\mathrm{~d}, J=8.5 \mathrm{~Hz}, 1 \mathrm{H})$ & 0.00 & & & \\
\hline 3 & $\begin{array}{l}1.26-1.20(\mathrm{~m}, 1 \mathrm{H}) \\
1.57-1.47(\mathrm{~m}, 1 \mathrm{H})\end{array}$ & $\begin{array}{l}1.24(\mathrm{~m}, 1 \mathrm{H}) \\
1.53(\mathrm{~m}, 1 \mathrm{H})\end{array}$ & & 30.4 & 30.4 & 0.0 \\
\hline 4 & $1.43-1.26(\mathrm{~m}, 2 \mathrm{H})$ & $\begin{array}{l}1.24(\mathrm{~m}, 1 \mathrm{H}) \\
1.31(\mathrm{~m}, 1 \mathrm{H}) \\
\end{array}$ & & 22.9 & 22.8 & +0.1 \\
\hline 5 & $1.43-1.26(\mathrm{~m}, 2 \mathrm{H})$ & $\begin{array}{l}1.35(\mathrm{~m}, 1 \mathrm{H}) \\
1.40(\mathrm{~m}, 1 \mathrm{H})\end{array}$ & & 29.1 & 29.1 & 0.0 \\
\hline 6 & $3.10-2.96(\mathrm{~m}, 2 \mathrm{H})$ & $3.03(\mathrm{~m}, 2 \mathrm{H})$ & & 38.5 & 38.5 & 0.0 \\
\hline $6-\mathrm{NH}$ & $7.99(\mathrm{t}, J=5.6 \mathrm{~Hz}, 1 \mathrm{H})$ & $7.98(\mathrm{~m}, 1 \mathrm{H})$ & +0.01 & & & \\
\hline $1^{\prime}$ & & & & 167.9 & 167.9 & 0.0 \\
\hline $2^{\prime}$ & $2.47-2.41(\mathrm{~m}, 2 \mathrm{H})$ & $\begin{array}{l}2.44(\mathrm{~m}, 1 \mathrm{H}) \\
2.48(\mathrm{~m}, 1 \mathrm{H}) \\
\end{array}$ & & 42.5 & 42.5 & 0.0 \\
\hline $3^{\prime}$ & 4.11-3.99 $(\mathrm{m}, 1 \mathrm{H})$ & $4.07(\mathrm{~m}, 1 \mathrm{H})$ & & 47.2 & 47.2 & 0.0 \\
\hline $4^{\prime}$ & $1.33-1.28(\mathrm{~m}, 3 \mathrm{H})$ & $1.30(\mathrm{~m}, 3 \mathrm{H})$ & & 21.0 & 21.0 & 0.0 \\
\hline $5^{\prime}$ & & & & 154.9 & 154.9 & 0.0 \\
\hline $1^{\prime \prime}$ & & & & 167.9 & 168.1 & -0.2 \\
\hline $2^{\prime \prime}$ & $2.41-2.33(\mathrm{~m}, 2 \mathrm{H})$ & $\begin{array}{l}2.37(\mathrm{~m}, 1 \mathrm{H}) \\
2.38(\mathrm{~m}, 1 \mathrm{H}) \\
\end{array}$ & & 42.3 & 42.3 & 0.0 \\
\hline $3^{\prime \prime}$ & 4.11-3.99 $(\mathrm{m}, 1 \mathrm{H})$ & $4.07(\mathrm{~m}, 1 \mathrm{H})$ & & 47.1 & 47.1 & 0.0 \\
\hline $4 "$ & $1.33-1.28(\mathrm{~m}, 3 \mathrm{H})$ & $1.30(\mathrm{~m}, 3 \mathrm{H})$ & & 21.0 & 21.0 & 0.0 \\
\hline $5^{\prime \prime}$ & & & & 154.9 & $153.6^{*}$ & +1.3 \\
\hline
\end{tabular}

DMSO- $d_{6}$ referenced to $2.50 \mathrm{ppm}\left({ }^{1} \mathrm{H}\right)$ and $39.52 \mathrm{ppm}\left({ }^{13} \mathrm{C}\right)$ for synthetic sample. ${ }^{*}$ This peak may have been misassigned, as the isonitrile carbons would be expected to have nearly identical chemical shifts. 


\section{H. Formation and Characterization of CU(I) COMPleX of Disonitriles}

\section{H1. Formation of Cu(I) Complex of Dissonitrile ACETate 4}<smiles>CC(=O)CC(=O)NCCCCC(COC(C)=O)NC(=O)CC(C)C</smiles>

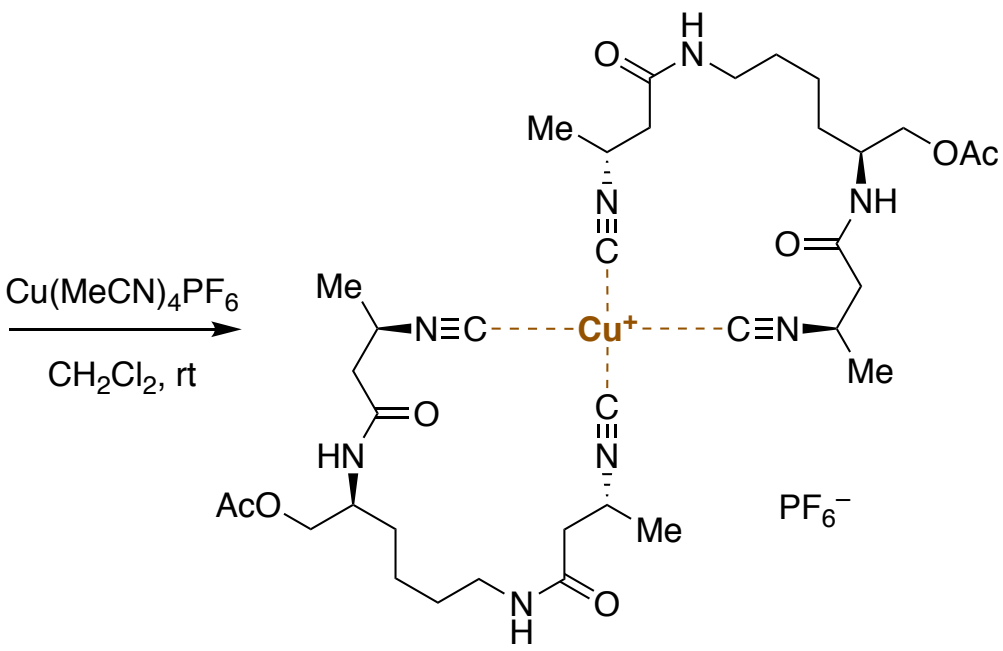

$(S)$-2,6-Bis $((R)-3$-isocyanobutanamido $)$ hexyl acetate $\cdot \mathbf{C u}(\mathbf{I}) \cdot \mathbf{P F}_{6}$ complex (S8). In a $15-\mathrm{mL}$ roundbottom flask in the glovebox were placed $(S)$-2,6-bis $((R)-3$-isocyanobutanamido)hexyl acetate (4) $(73.5 \mathrm{mg}, 0.202 \mathrm{mmol}, 2.1$ equiv) and tetrakis(acetonitrile)copper(I)hexafluorophosphate $(35.8 \mathrm{mg}, 1.0$ equiv). The flask was sealed with a septum and removed from the glovebox, then $\mathrm{CH}_{2} \mathrm{Cl}_{2}(2.5 \mathrm{~mL})$ was added. The mixture was stirred at $\mathrm{rt}$ for $3 \mathrm{~h}$, then $\mathrm{Et}_{2} \mathrm{O}(3$ $\mathrm{mL}$ ) was added dropwise, leading to precipitation of a white solid. The mixture was filtered and the filter cake was rinsed with $\mathrm{Et}_{2} \mathrm{O}(3 \times 3 \mathrm{~mL})$ to afford the solid copper complex $\mathbf{S 8}$ as a white solid (78.0 mg, 87\% yield), which was characterized without further purification.

IR (ZnSe, film): 3317 (w), 3294 (w), 2945 (w), 2184 (m), 1737 (m), 1651 (s), 1541 (m), 1452 $(\mathrm{w}), 1234(\mathrm{~m}), 1045(\mathrm{w}), 840(\mathrm{~s}) \mathrm{cm}^{-1} .{ }^{1}$ H-NMR $\left(600 \mathrm{MHz}, \mathrm{DMSO}-d_{6}\right): \delta 8.10(\mathrm{dd}, J=6.7,4.4$ $\mathrm{Hz}, 2 \mathrm{H}), 8.00$ (d, $J=8.4 \mathrm{~Hz}, 2 \mathrm{H}), 4.43-4.28(\mathrm{~m}, 4 \mathrm{H}), 4.11-3.93$ (m, 4H), 3.86 (dd, $J=10.8$, $6.2 \mathrm{~Hz}, 2 \mathrm{H}), 3.27(\mathrm{dq}, J=12.9,6.4 \mathrm{~Hz}, 2 \mathrm{H}), 2.89$ (h, $J=5.3 \mathrm{~Hz}, 2 \mathrm{H}), 2.57-2.51(\mathrm{~m}, 4 \mathrm{H}), 2.50$ $-2.46(\mathrm{~m}, 2 \mathrm{H}), 2.41(\mathrm{dd}, J=14.7,10.6 \mathrm{~Hz}, 2 \mathrm{H}), 2.00(\mathrm{~s}, 6 \mathrm{H}), 1.55-1.48(\mathrm{~m}, 2 \mathrm{H}), 1.47-1.41$ $(\mathrm{m}, 2 \mathrm{H}), 1.39(\mathrm{~d}, J=6.7 \mathrm{~Hz}, 12 \mathrm{H}), 1.35-1.21(\mathrm{~m}, 8 \mathrm{H}) .{ }^{13} \mathbf{C}-\mathbf{N M R}\left(151 \mathrm{MHz}, \mathrm{DMSO}-d_{6}\right):{ }^{18}$ $\delta 170.3,167.9,65.9,50.049 .2,47.4,41.4,41.3,37.7,29.2,28.0,21.8,20.7,20.5,20.2$. ESI-MS: Calcd for $\mathrm{C}_{36} \mathrm{H}_{56} \mathrm{~N}_{8} \mathrm{O}_{8} \mathrm{Cu}^{63}\left([\mathrm{M}]^{+}\right): 791.3517$, Found: $791.3527 ; \mathrm{C}_{36} \mathrm{H}_{56} \mathrm{~N}_{8} \mathrm{O}_{8} \mathrm{Cu}^{65}$ $\left([\mathrm{M}]^{+}\right):$793.3499, Found: 793.3514.

\footnotetext{
${ }^{18}$ The ${ }^{13} \mathrm{C}$ NMR signal of the isonitrile carbon coordinated with $\mathrm{Cu}$ is difficult to observe due to ${ }^{63 / 65} \mathrm{Cu}$-quadrupole broadening; see: Walther, M.; Jung, C. M.; Bergmann, R.; Pietzsch, J.; Rode, K.; Fahmy, K.; Mirtschink, P.; Stehr, S.; Heintz, A.; Wunderlich, G.; Kraus, W.; Pietzsch, H.-J.; Kropp, J.; Deussen, A.; Spies, H. Bioconjugate Chem. 2007, 18 (1), 216.
} 
H2. Comparison of NMR and IR Spectra of Free Ditsonitrile Acetate 4 and CORRESPONDING CU(I) COMPLEX S8

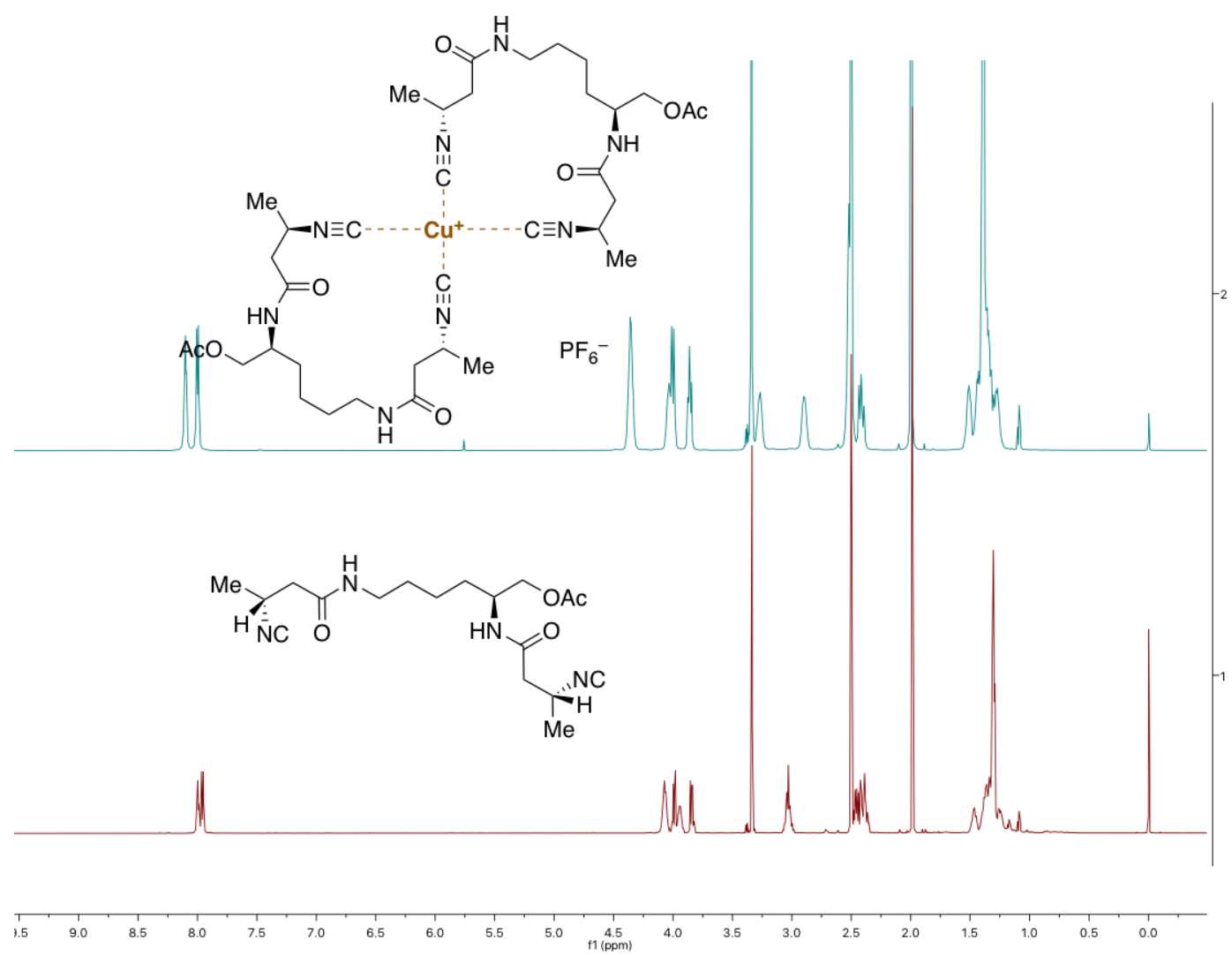

Figure S8. Stacked ${ }^{1} \mathrm{H}-\mathrm{NMR}$ spectra of diisonitrile acetate 4 (bottom) and corresponding $\mathrm{Cu}(\mathrm{l})$ complex S8 (top). 

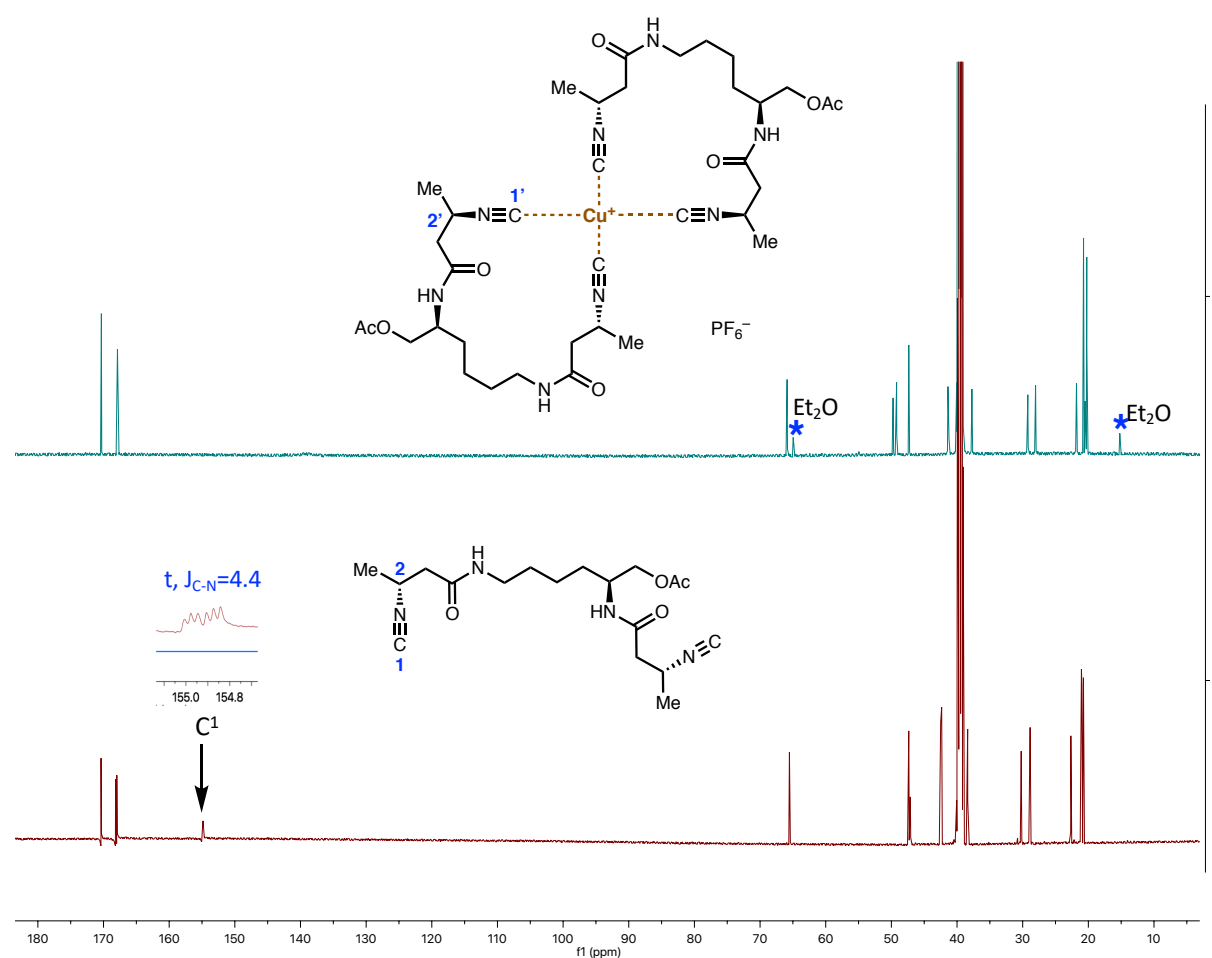

Figure S9. Stacked ${ }^{13} \mathrm{C}$-NMR spectra of diisonitrile acetate 4 and corresponding $\mathrm{Cu}(\mathrm{I})$ complex S8. The ${ }^{13} \mathrm{C}$-NMR signal for $\mathrm{C}^{1}{ }^{\prime}$ in the complex $\mathbf{S} 8$ is difficult to observe due to ${ }^{63 / 65} \mathrm{Cu}$-quadrupole coupling.

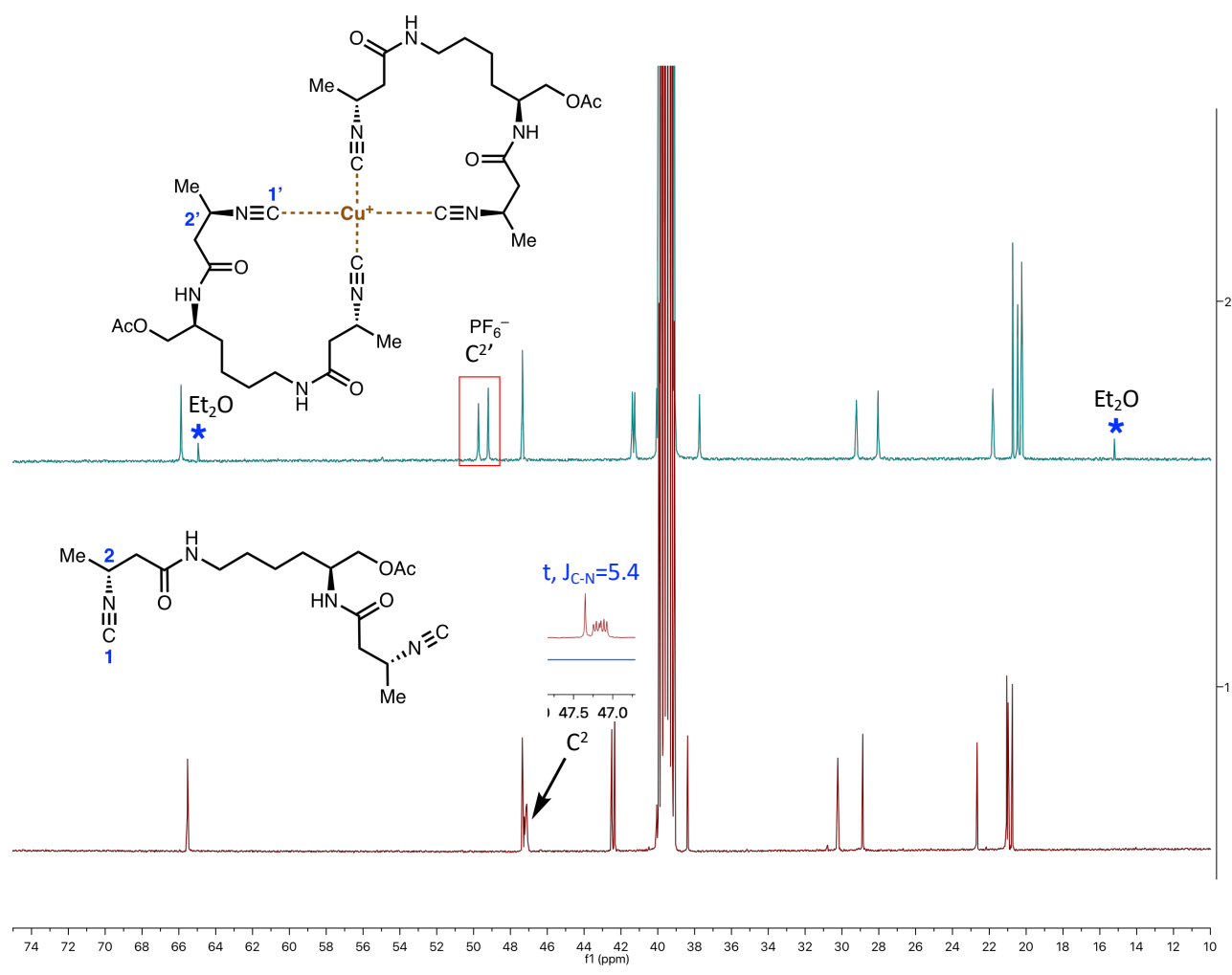

Figure S10. Expanded regions of stacked ${ }^{13} \mathrm{C}$-NMR spectra of diisonitrile acetate 4 and corresponding $\mathrm{Cu}(\mathrm{I})$ complex S8. 

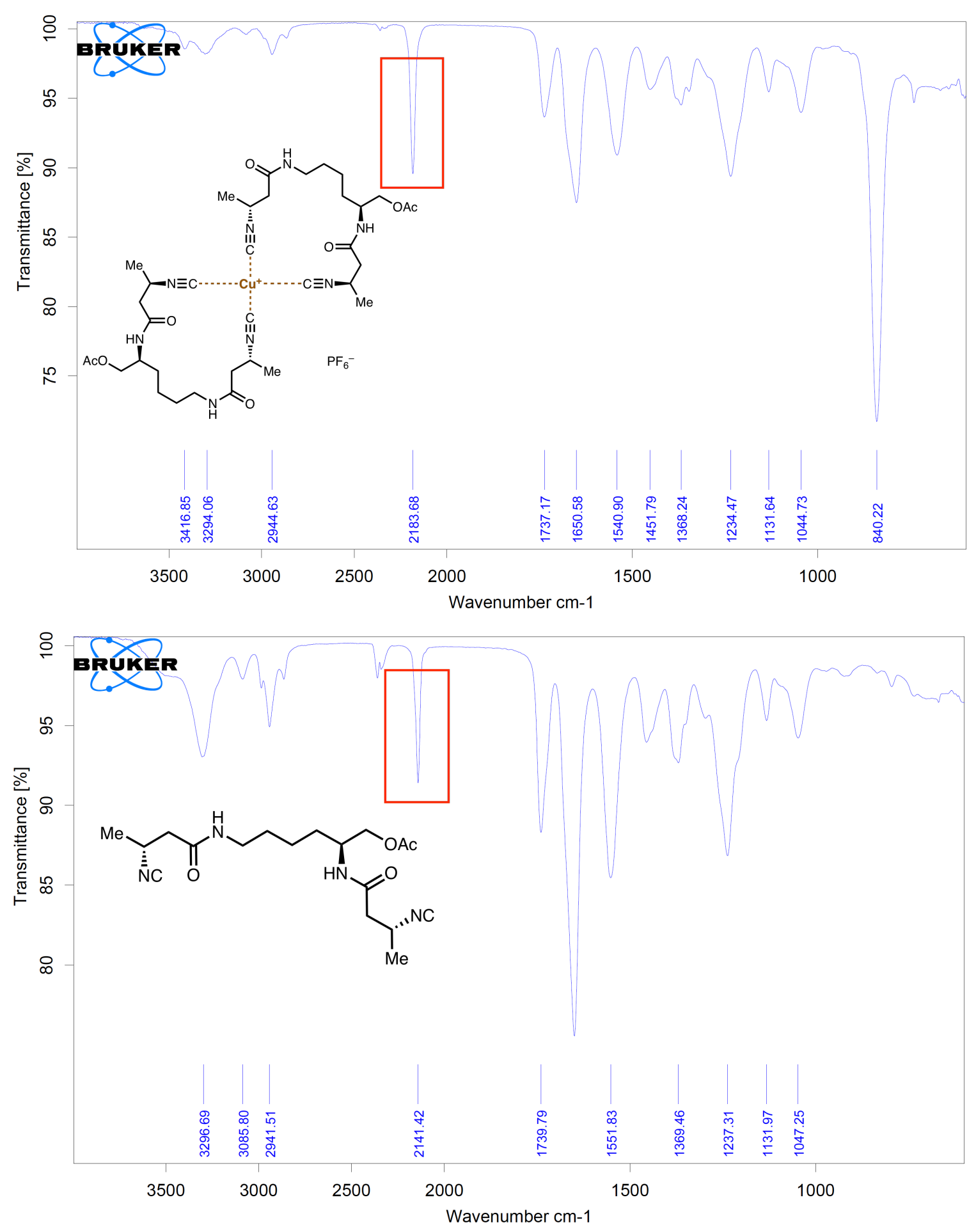

Figure S11. IR spectra of diisonitrile acetate 4 (bottom) and corresponding Cu(I) complex S8 (top). The isonitrile CN stretch frequency (red box) shifts from $2141 \mathrm{~cm}^{-1}$ to $2184 \mathrm{~cm}^{-1}$. 


\section{H3. ATteMPTEd Formation OF Cu(I) COMPLEXES OF SF2768 ((2L,5S)-1) AND 5-epi-SF2768 $((2 \mathrm{~L}, 5 R)-1)$}

We attempted to form the $\mathrm{Cu}(\mathrm{I})$ complex of SF2768 (2L,5S-1) as well as 5-epi-SF2768 $(2 \mathrm{~L}, \mathbf{5 R}-\mathbf{1})$. However, the ${ }^{1} \mathrm{H}-\mathrm{NMR}$ spectra of the resulting complexes were too broad to assign any peaks, possibly due to the mixture of $\mathrm{C} 1$-anomers.

Formation of $\mathrm{Cu}(I)$ complex of SF2768 $(2 L, 5 S-1)$

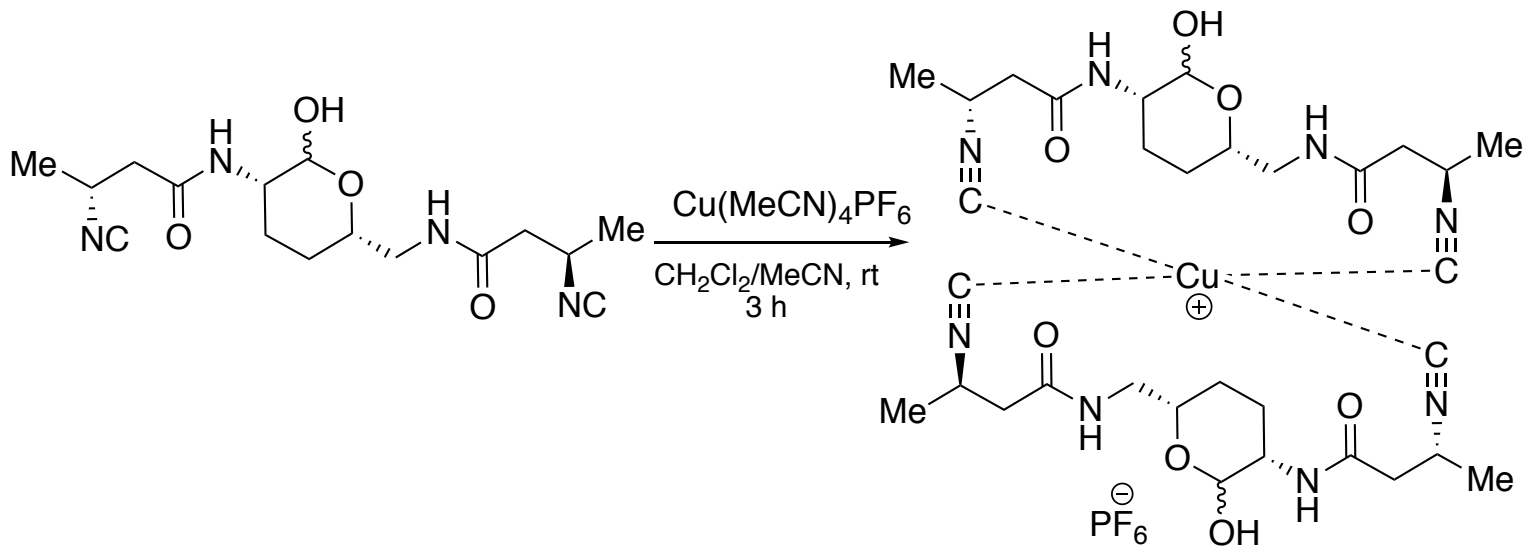

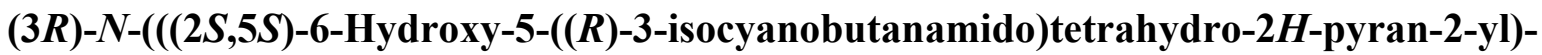
methyl)-3-isocyanobutanamide $\cdot \mathbf{C u}(\mathbf{I}) \cdot \mathbf{P F}_{6}$ complex (S9). In a flame-dried $13 \times 100 \mathrm{~mm}$ test tube in a glovebox were placed $(3 R)-N-(((2 S, 5 S)$-6-hydroxy-5- $((R)-3$-isocyanobutanamido)tetrahydro-2H-pyran-2-yl)methyl)-3-isocyanobutanamide (SF2768, 2L,5S-1) $(7.8 \mathrm{mg}, 0.0232 \mathrm{mmol}$, 2.0 equiv) and tetrakis(acetonitrile)copper(I)hexafluorophosphate $(4.32 \mathrm{mg}, 0.0116 \mathrm{mmol}, 1.0$ equiv). The test tube was sealed with a septum and removed from the glovebox, followed by addition of $\mathrm{CH}_{2} \mathrm{Cl}_{2}(0.4 \mathrm{~mL})$ and $\mathrm{MeCN}(0.8 \mathrm{~mL})$. The mixture was stirred at $\mathrm{rt}$ for $3 \mathrm{~h}$ and concentrated by rotary evaporation to afford the SF2768 copper complex S9 as a colorless oil. (10.2 mg, quant. yield), which was characterized without further purification. HR-ESI-MS analysis indicated the mass of the desired copper complex and a change in the chemical shift of the protons alpha to the isonitrile groups was also observed by ${ }^{1} \mathrm{H}-\mathrm{NMR}$, although complete assignments were precluded by the broad peak shapes.

ESI-MS: Calcd for $\mathrm{C}_{32} \mathrm{H}_{48} \mathrm{~N}_{8} \mathrm{O}_{8}\left[{ }^{63} \mathrm{Cu}\right]\left([\mathrm{M}]^{+}\right)$: 735.2891, Found: 735.2864. Calcd for $\mathrm{C}_{32} \mathrm{H}_{48} \mathrm{~N}_{8} \mathrm{O}_{8}\left[{ }^{65} \mathrm{Cu}\right]\left([\mathrm{M}]^{+}\right): 737.2873$, Found: 737.2844. 

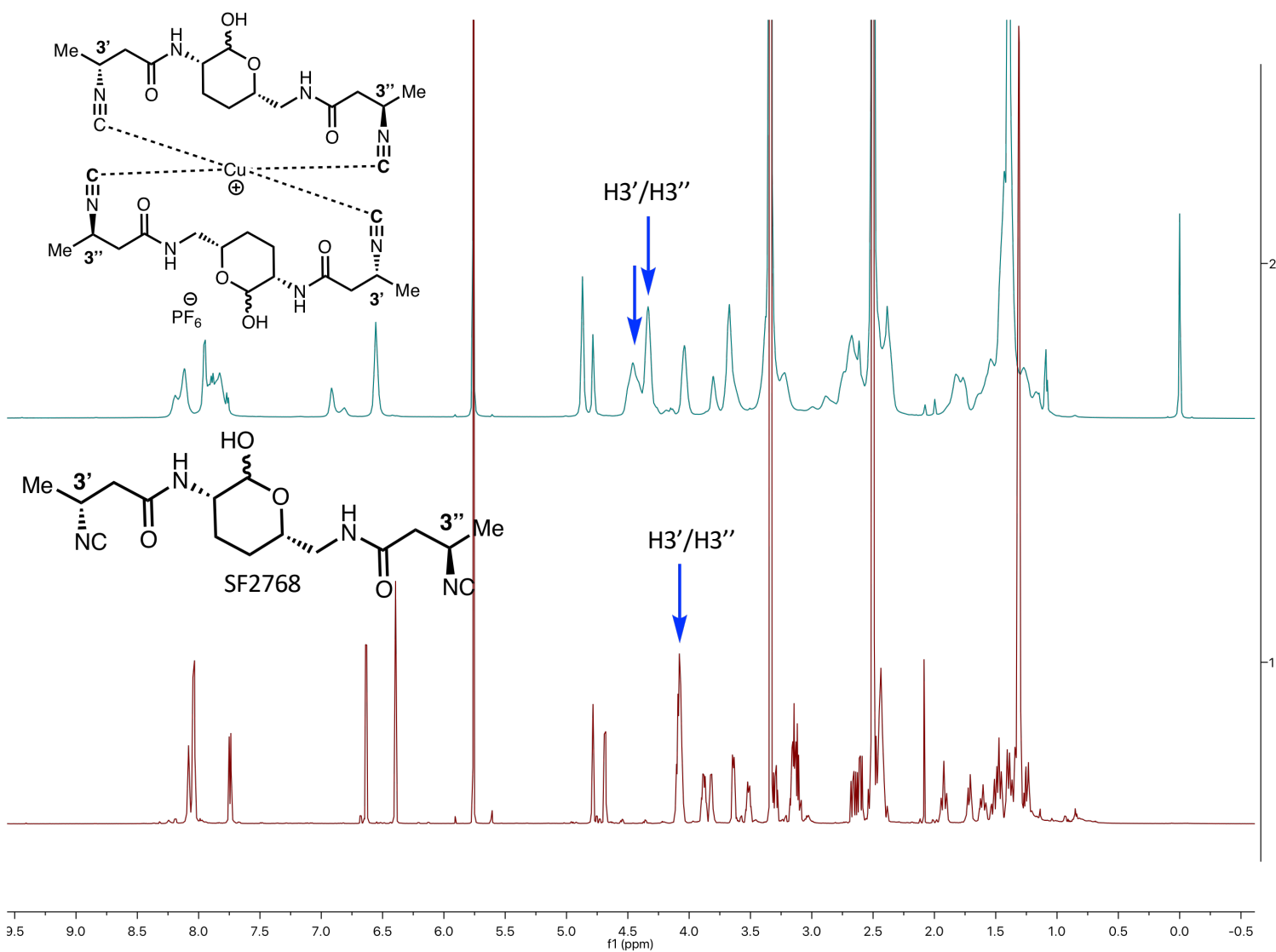

Figure S12. Stacked ${ }^{1} \mathrm{H}-\mathrm{NMR}$ of diisonitrile SF2768 (2L,5S-1) and corresponding $\mathrm{Cu}(\mathrm{I})$ complex (S9). $\mathrm{H}^{\prime}$ ' and $\mathrm{H}^{\prime \prime}$ signals for copper complex assigned based on HSQC spectrum (see below).

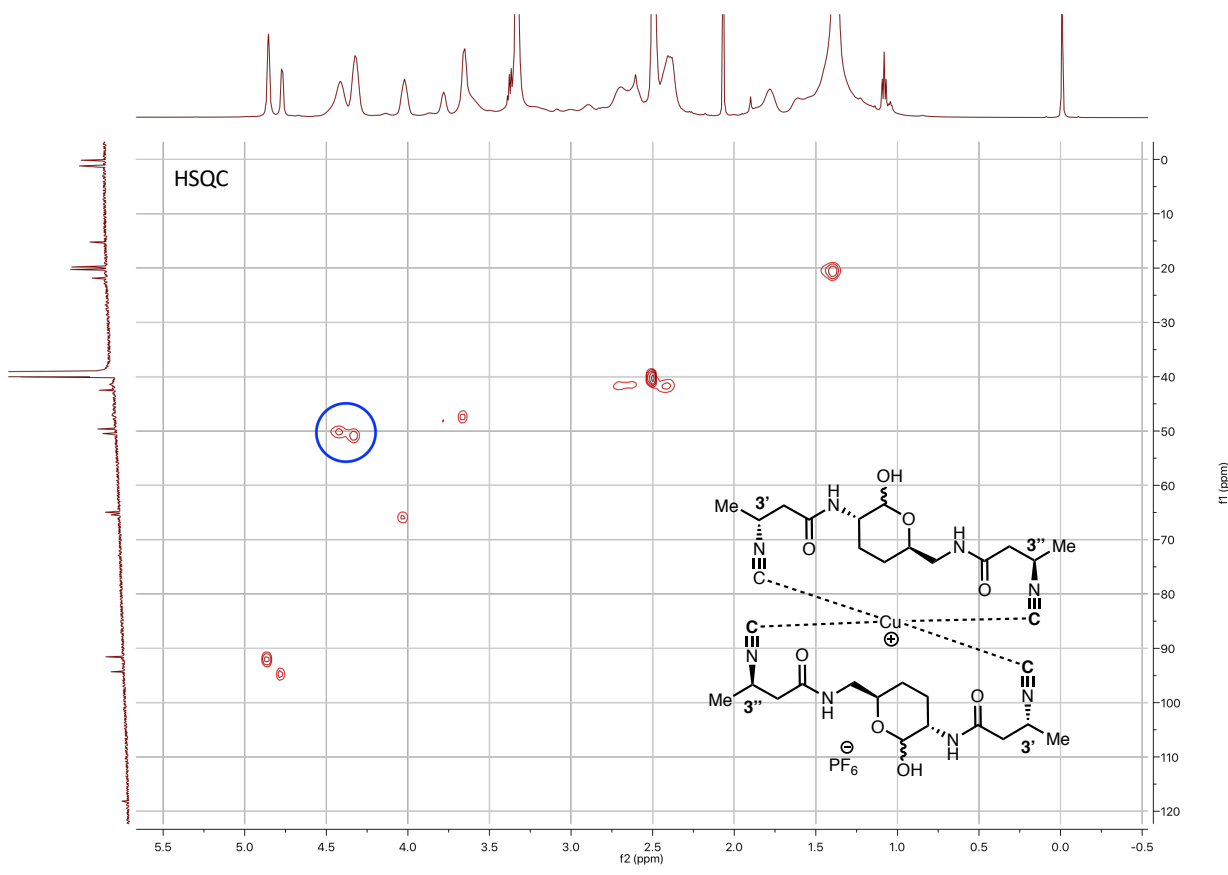

Figure S13. HSQC spectrum of $\mathrm{Cu}(\mathrm{I})$ complex (S9) of diisonitrile SF2768 (2L,5S-1) used for assignment of $\mathrm{H}^{\prime}$ and $\mathrm{H}^{\prime \prime}$ resonances. 
Formation of $\mathrm{Cu}(I)$ complex of 5-epi-SF2768 (2L,5R-1)<smiles>CC(C)CC(=O)N[C@H]1CC[C@@H](CNC(=O)C[C@H](C)N)O[C@@H]1O</smiles>

(3R)-N-(((2R,5S)-6-Hydroxy-5-((R)-3-isocyanobutanamido)tetrahydro-2H-pyran-2-yl)methyl)-3-isocyanobutanamide $\cdot \mathbf{C u}(\mathbf{I}) \cdot \mathbf{P F}_{6}$ complex (S10). In a flame-dried $13 \times 100 \mathrm{~mm}$ test tube in a glovebox were placed $(3 R)-N-((2 R, 5 S)-6$-hydroxy-5- $((R)-3$-isocyanobutanamido)tetrahydro-2H-pyran-2-yl)methyl)-3-isocyanobutanamide (5-epi-SF2768, 2L,5R-1) (7.3 mg, 0.0217 mmol, 2.0 equiv) and tetrakis(acetonitrile)copper(I)hexafluorophosphate (4.04 mg, $0.0109 \mathrm{mmol}$, 1.0 equiv). The test tube was sealed a septum and removed from the glovebox, followed by addition of $\mathrm{CH}_{2} \mathrm{Cl}_{2}(0.3 \mathrm{~mL}), \mathrm{MeCN}(0.3 \mathrm{~mL})$, and $\mathrm{MeOH}(0.3 \mathrm{~mL})$. The mixture was stirred at $\mathrm{rt}$ for $3 \mathrm{~h}$, then concentrated by rotary evaporation to afford 5-epi-SF2768. copper complex S10 as a white solid $(9.60 \mathrm{mg}$, quant. yield), which was characterized without further purification. HR-ESI-MS analysis indicated the mass of the desired copper complex and a change in the chemical shift of the protons alpha to the isonitrile groups was also observed by ${ }^{1} \mathrm{H}-\mathrm{NMR}$, although complete assignments were precluded by the broad peak shapes.

ESI-MS: Calcd for $\mathrm{C}_{32} \mathrm{H}_{48} \mathrm{~N}_{8} \mathrm{O}_{8}\left[{ }^{63} \mathrm{Cu}\right]\left([\mathrm{M}]^{+}\right)$: 735.2891, Found: 735.2872. Calcd for $\mathrm{C}_{32} \mathrm{H}_{48} \mathrm{~N}_{8} \mathrm{O}_{8}\left[{ }^{65} \mathrm{Cu}\right]\left([\mathrm{M}]^{+}\right): 737.2873$, Found: 737.2893. 

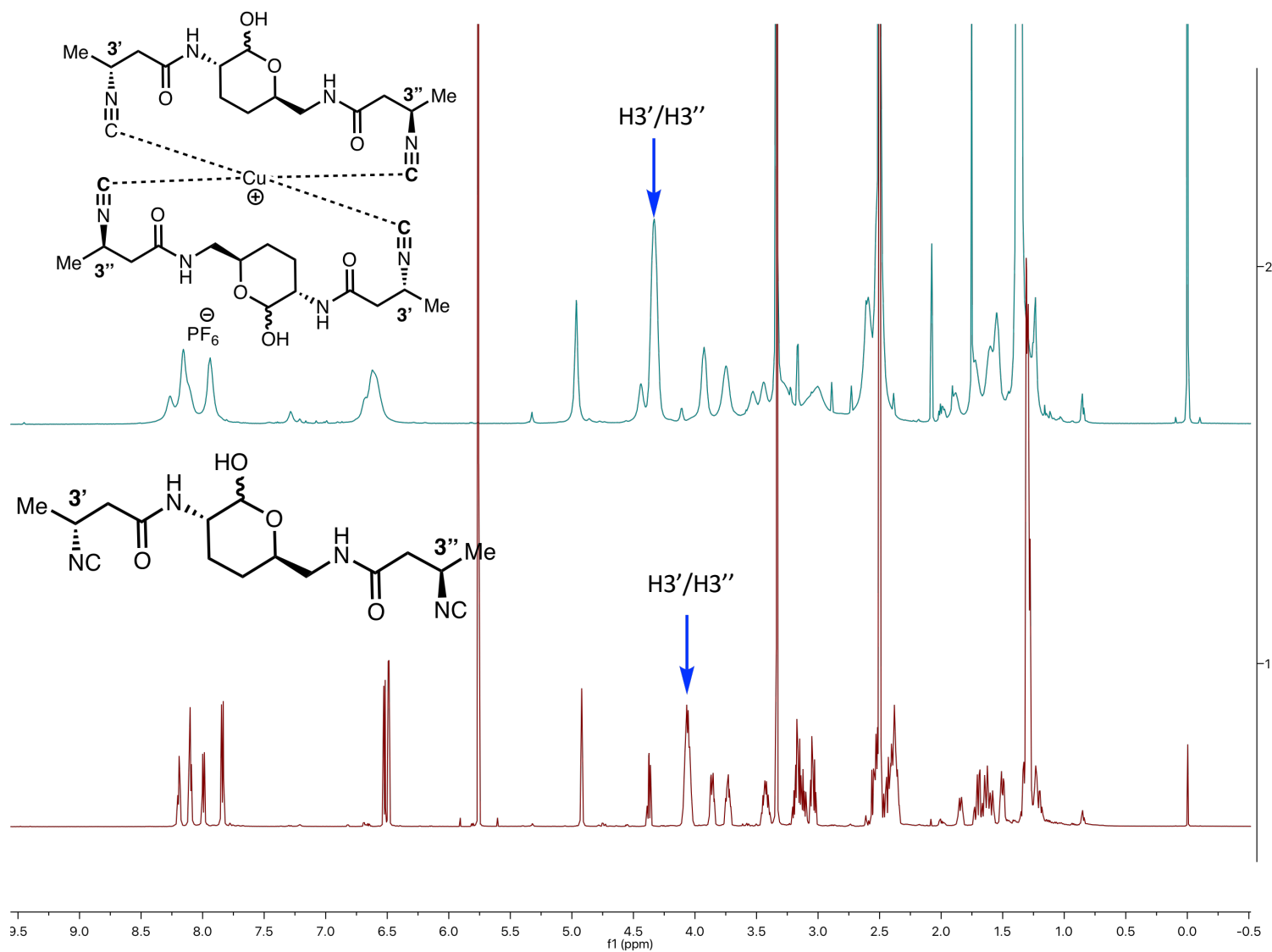

Figure S14. Stacked ${ }^{1} \mathrm{H}$-NMR of diisonitrile 5-epi-SF2768 (2L,5R-1) and corresponding $\mathrm{Cu}(\mathrm{I})$ complex (S10). $\mathrm{H}^{\prime}$ ' and $\mathrm{H}^{\prime \prime}$ signals for $\mathrm{Cu}$ complex assigned based on HSQC spectrum (see below).

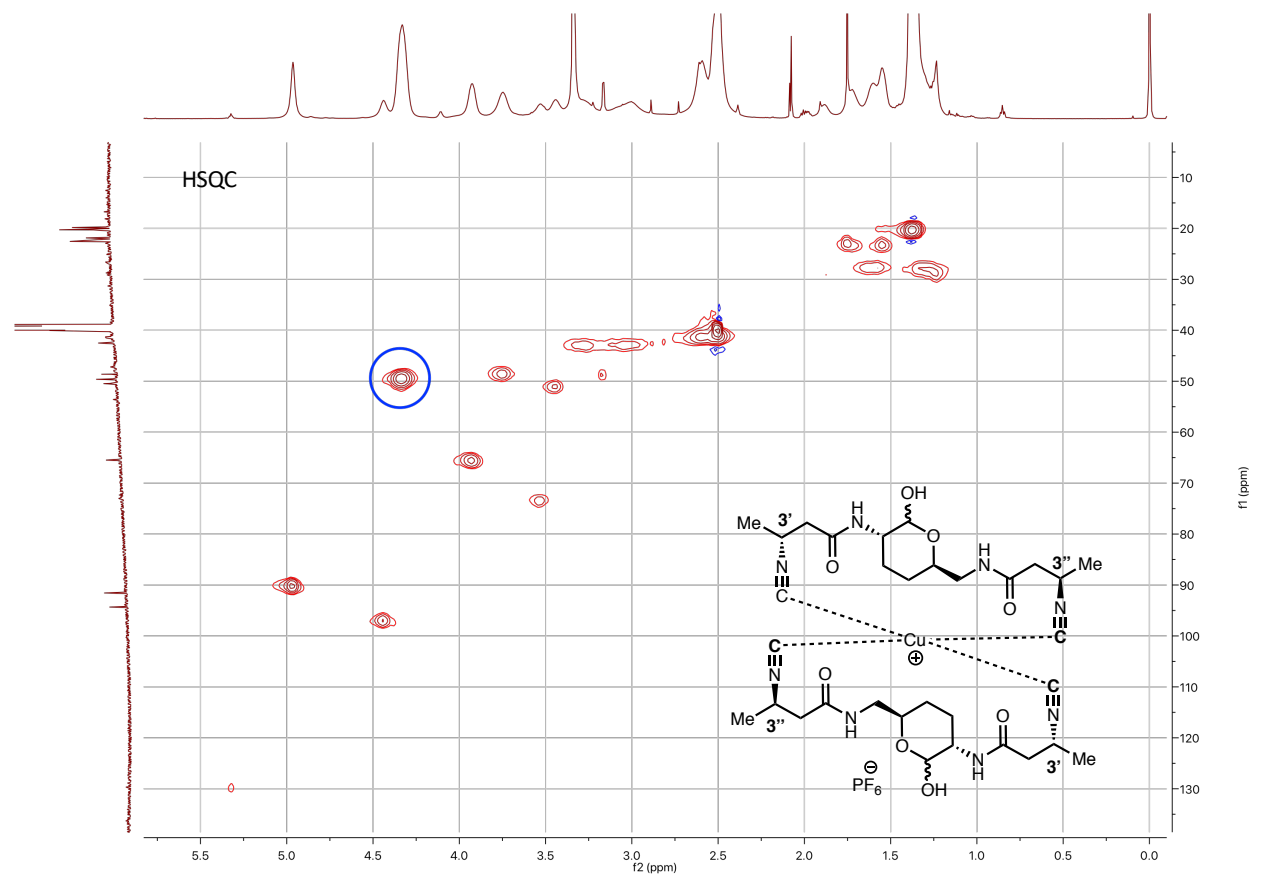

Figure S15. HSQC spectrum of $\mathrm{Cu}(\mathrm{I})$ complex (S10) of diisonitrile 5-epi-SF2768 (2L,5R-1) used for assignment of $\mathrm{H}^{\prime}$ ' and $\mathrm{H}^{\prime \prime}$ "resonances. 


\section{I. ${ }^{1}$ H-NMR TITRATION OF DiISONITRILE ACETATE 4 WITH CU(I)}

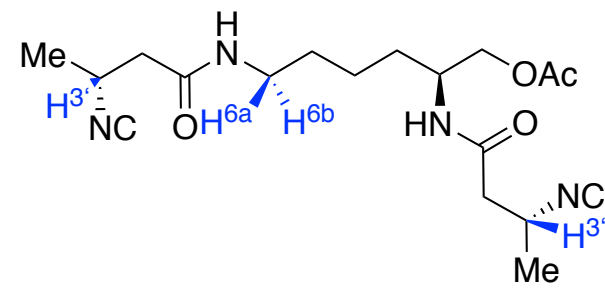

4

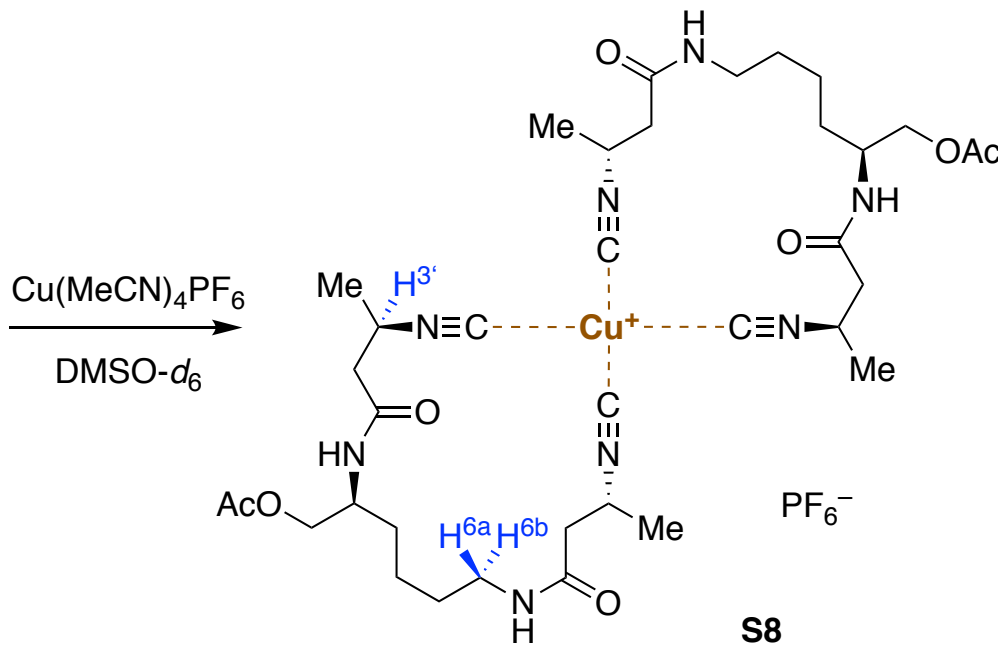

Individual samples were prepared with the same final concentration of diisonitrile acetate 4 [18.81 mM] and varying concentrations of $\mathrm{Cu}(\mathrm{I})$ for ${ }^{1} \mathrm{H}-\mathrm{NMR}$ analysis. In a $20-\mathrm{mL}$ flame-dried vial, diisonitrile acetate $4\left(48.0 \mathrm{mg}, 0.1317 \mathrm{mmol}\right.$ ) was dissolved in $6.0 \mathrm{~mL} \mathrm{DMSO}-d_{6}$ (from ampule). Aliquots of $0.6 \mathrm{~mL}$ of this solution were transferred into 9 separate NMR tubes, providing $4.8 \mathrm{mg}, 0.01317 \mathrm{mmol}$ of diisonitrile acetate 4 in each. $\mathrm{Cu}(\mathrm{MeCN})_{4} \mathrm{PF}_{6}$ was weighed outside the glovebox ${ }^{19}$ then the vial was placed under vacuum and filled with Ar three times, followed by addition of varying amounts DMSO- $d_{6}$ as indicated below. The color of the $\mathrm{Cu}$ solution slowly changed to green, and after $3 \mathrm{~min}, 100 \mu \mathrm{L}$ of each solution was transferred to the corresponding NMR tube containing diisonitrile acetate 4. The mixture was shaken well, then the ${ }^{1} \mathrm{H}-\mathrm{NMR}$ spectrum recorded after $30 \mathrm{~min}$.

\begin{tabular}{|l|l|l|l|l|}
\hline sample & final equiv. $\mathbf{C u}(\mathbf{l})$ & $\begin{array}{l}\text { mass } \\
\text { Cu(MeCN})_{4} \text { PF }_{6}\end{array}$ & $\begin{array}{l}\text { total volume } \\
\text { DMSO- } \boldsymbol{d}_{6}\end{array}$ & $\begin{array}{l}\text { volume DMSO- } \boldsymbol{d}_{6} \\
\text { transferred }\end{array}$ \\
\hline 1 & 0 & 0 & $0.2000 \mathrm{~mL}$ & $100 \mu \mathrm{L}$ \\
\hline 2 & $0.1(0.0013 \mathrm{mmol})$ & $5.0 \mathrm{mg}$ & $1.0186 \mathrm{~mL}$ & $100 \mu \mathrm{L}$ \\
\hline 3 & $0.2(0.0026 \mathrm{mmol})$ & $5.0 \mathrm{mg}$ & $0.5093 \mathrm{~mL}$ & $100 \mu \mathrm{L}$ \\
\hline 4 & $0.3(0.0039 \mathrm{mmol})$ & $5.0 \mathrm{mg}$ & $0.3395 \mathrm{~mL}$ & $100 \mu \mathrm{L}$ \\
\hline 5 & $0.4(0.0052 \mathrm{mmol})$ & $5.0 \mathrm{mg}$ & $0.2547 \mathrm{~mL}$ & $100 \mu \mathrm{L}$ \\
\hline 6 & $0.5(0.0065 \mathrm{mmol})$ & $5.0 \mathrm{mg}$ & $0.2037 \mathrm{~mL}$ & $100 \mu \mathrm{L}$ \\
\hline 7 & $0.6(0.0078 \mathrm{mmol})$ & $10.0 \mathrm{mg}$ & $0.3396 \mathrm{~mL}$ & $100 \mu \mathrm{L}$ \\
\hline 8 & $0.8(0.0104 \mathrm{mmol})$ & $10.0 \mathrm{mg}$ & $0.2546 \mathrm{~mL}$ & $100 \mu \mathrm{L}$ \\
\hline 9 & $1.0(0.0132 \mathrm{mmol})$ & $10.0 \mathrm{mg}$ & $0.2037 \mathrm{~mL}$ & $100 \mu \mathrm{L}$ \\
\hline
\end{tabular}

${ }^{19} \mathrm{Cu}(\mathrm{MeCN})_{4} \mathrm{PF}_{6}$ was stored in a glovebox, but could be weighed outside of the glovebox in a short period of time. The stability of this complex has been discussed previously: Wang, F. , Chen, P. and Liu, G. (2017).

Tetrakis(acetonitrile)copper(I) Hexafluorophosphate. In Encyclopedia of Reagents for Organic Synthesis. doi:10.1002/047084289X.rn02002 


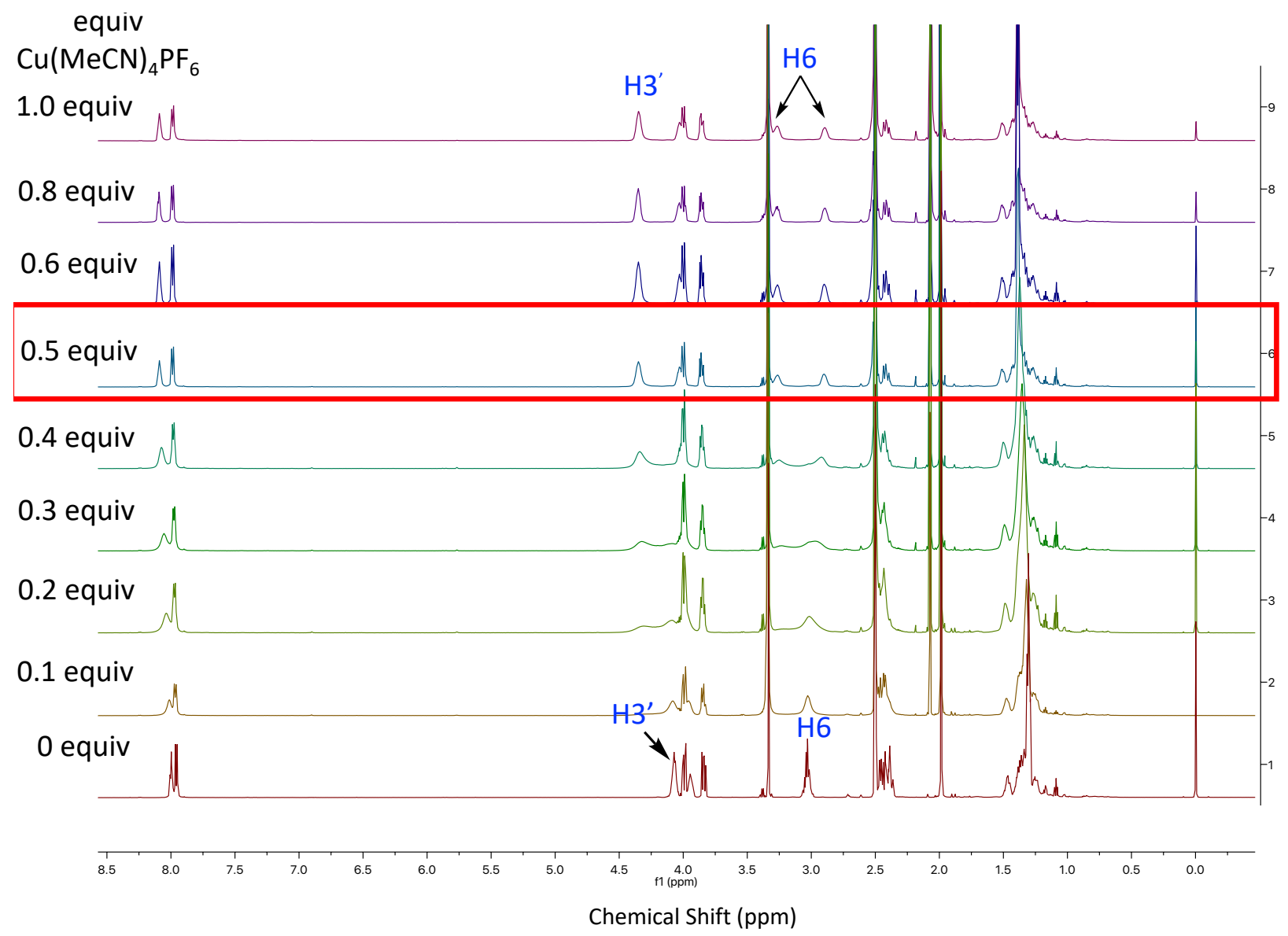

Figure S16. Stacked ${ }^{1} \mathrm{H}-\mathrm{NMR}$ titration spectra of diisonitrile acetate 4 with varying amounts of $\mathrm{Cu}(\mathrm{MeCN})_{4} \mathrm{PF}_{6}$ in DMSO- $d_{6}$. 


\section{J. UV-Vis Competitive Titration EXPERIMENTS OF CU(I) COMPLEXES WiTH BCS}

\section{Preparation of $\mathrm{Cu}(\mathrm{BCS})_{2}$ stock solution}

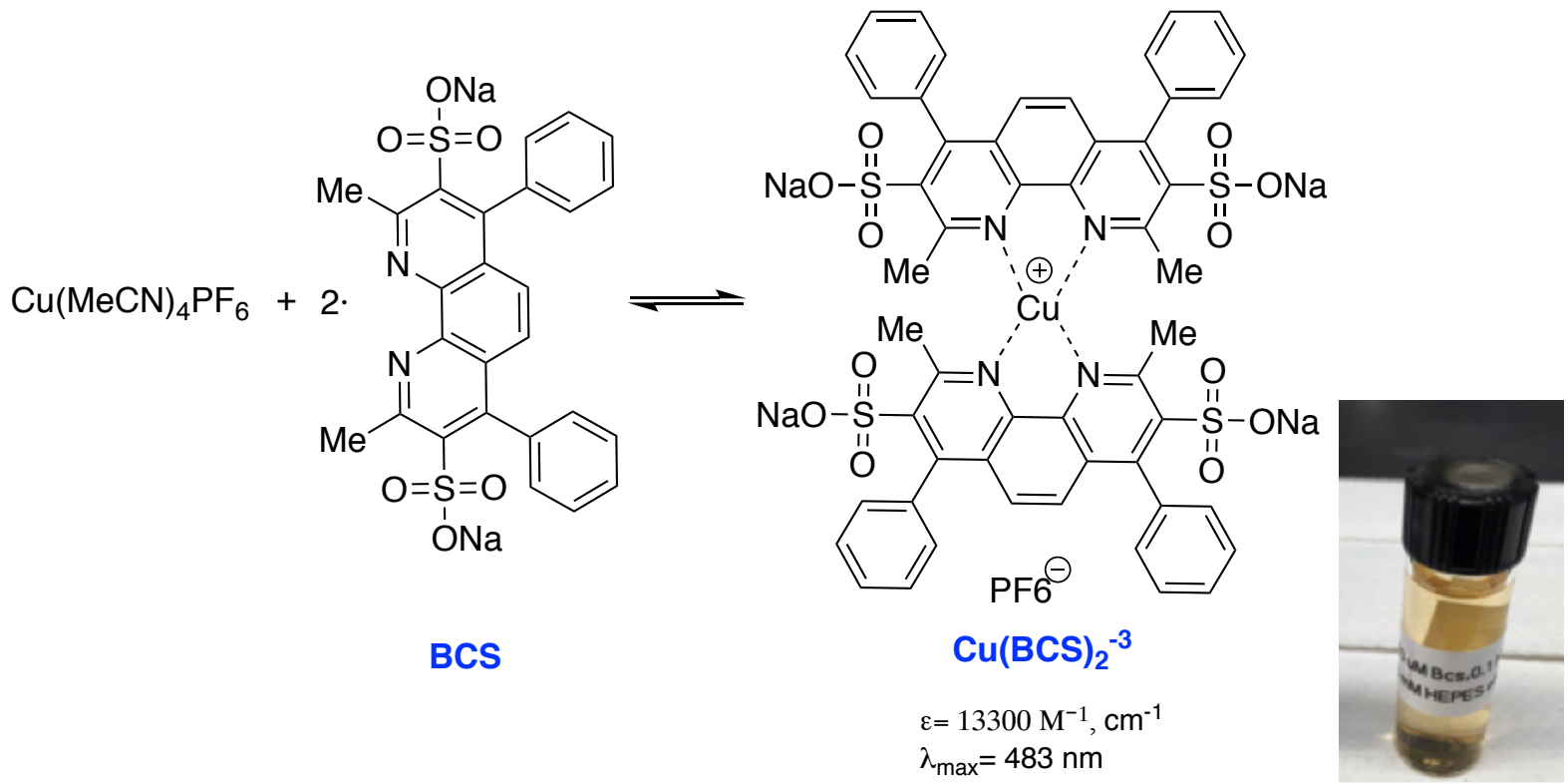

A solution of $25 \mathrm{mM}$ HEPES buffer $\left(0.1 \mathrm{M} \mathrm{NaCl}, \mathrm{pH} 7.2, \mathrm{MeCN}: \mathrm{H}_{2} \mathrm{O} \mathrm{v} / \mathrm{v}=1: 10\right)$ was prepared by diluting commercial 1.0 M HEPES buffer (Corning) with Milli-Q water and degassed MeCN. The diluted HEPES buffer was then degassed and deoxygenated using a sonicator and Ar. An aliquot of $20 \mu \mathrm{L}$ of $10 \mathrm{mM}$ stock solution of Bathocuproinedisulfonic acid disodium salt (BCS) in degassed $25 \mathrm{mM}$ HEPES buffer and $4 \mu \mathrm{L}$ of $20 \mathrm{mM}$ stock solution of $\mathrm{Cu}(\mathrm{MeCN})_{4} \mathrm{PF}_{6}$ in degassed MeCN was mixed and diluted using $25 \mathrm{mM}$ HEPES buffer to the final volume of $2 \mathrm{~mL}$.

\section{Competitive titration experiments of $\mathrm{Cu}(\mathrm{BCS})_{2}$ with diisonitrile acetate 4}

Stock solutions of $1 \mathrm{mM}, 1.5 \mathrm{mM}, 2 \mathrm{mM}, 2.5 \mathrm{mM}, 3 \mathrm{mM}, 3.5 \mathrm{mM}, 4 \mathrm{mM}, 5 \mathrm{mM}$, and $6 \mathrm{mM}$ diisonitrile acetate 4 were prepared in degassed, anhydrous DMSO. Aliquots of $1.2 \mu \mathrm{L}$ of each stock solution of diisonitrile acetate 4 were added to aliquots of $118.8 \mu \mathrm{L}$ of the $\mathrm{Cu}(\mathrm{BCS})_{2}$ solution. Samples of $100 \mu \mathrm{L}$ of each final solution were analyzed by UV-Vis. ${ }^{20}$ The concentration of $\mathrm{Cu}(\mathrm{BCS})_{2}$ remaining in each sample was calculated using the Beer-Lambert law $\left(\lambda_{\max }=483 \mathrm{~nm}, \varepsilon=13300 \mathrm{M}^{-1} \mathrm{~cm}^{-1}\right){ }^{21}$ We confirmed separately that the $\mathrm{Cu}(\mathrm{I})$ complex with diisonitrile acetate 4 has no absorbance at $483 \mathrm{~nm}$.

\footnotetext{
${ }^{20}$ Using the Costar 96-well black, clear-bottom multiplate for the UV-Vis measurements, raw absorbance was recorded and the pathlength of the $100 \mu \mathrm{L}$ solution was $0.3 \mathrm{~cm}$. see: http://sites.psu.edu/msproteomics/wpcontent/uploads/sites/7129/2014/05/User-Manual-SpectraMax-M3-M4-M5-M5e-.pdf

${ }^{21}$ Xiao, Z.; Loughlin, F.; George, G. N.; Howlett, G. J.; Wedd, A. G. J. Am. Chem. Soc. 2004, 126 (10), 3081.
} 

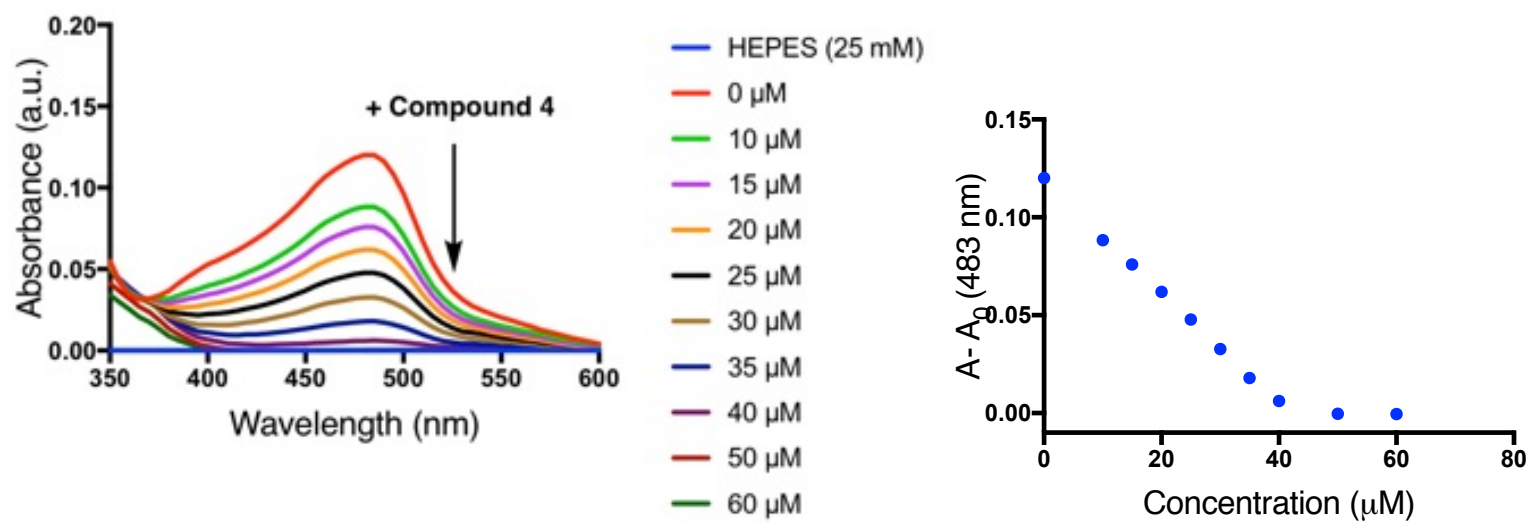

Figure S17. UV-Vis titration of $\mathrm{Cu}(\mathrm{BCS})_{2}$ with various equivalents of diisonitrile acetate 4.

Table S9. Results of $\mathrm{Cu}(\mathrm{BCS})_{2}$ titration experiments with diisonitrile acetate 4.

\begin{tabular}{|l|l|l|l|l|l|}
\hline $\begin{array}{l}\text { total }[\mathrm{BCS}] \\
(\mu \mathrm{M})\end{array}$ & $\begin{array}{l}\text { total }[\mathrm{Cu}] \\
(\mu \mathrm{M})\end{array}$ & $\begin{array}{l}\text { total } \\
{[(\mu \mathrm{M})}\end{array}$ & $\mathbf{A}_{483}$ & $\begin{array}{l}\text { calculated } \\
{\left[\mathrm{Cu}(\mathrm{BCS})_{2}\right]} \\
(\mu \mathrm{M})^{a}\end{array}$ & $\begin{array}{l}\text { calculated } \\
{[\mathrm{Cu} \cdot 4 \cdot \mathrm{BCS}]+} \\
{\left[\mathrm{Cu} \cdot(\mathbf{4})_{2}\right](\mu \mathrm{M})^{b}}\end{array}$ \\
\hline 100 & 40 & 0 & 0.1201 & 30.1 & 0 \\
\hline & & 0.0883 & 22.13 & 7.97 \\
\hline & & 10 & 0.0759 & 19.02 & 11.08 \\
\hline & & 20 & 0.062 & 15.54 & 14.56 \\
\hline & & 0.0478 & 11.98 & 18.12 \\
\hline & & 30 & 0.0327 & 8.2 & 21.9 \\
\hline & & 35 & 0.018 & 4.51 & 25.59 \\
\hline & & 40 & 0.0062 & 1.55 & 28.55 \\
\hline
\end{tabular}

${ }^{a}$ Based on Beer-Lambert law using $\lambda_{\max }=483 \mathrm{~nm}, \varepsilon=13300 \mathrm{M}^{-1} \mathrm{~cm}^{-1}$; ${ }^{b}$ Based on subtraction of calculated $\left[\mathrm{Cu}(\mathrm{BCS})_{2}\right]$ concentration from initial concentration $(30.1 \mu \mathrm{M})$.

The overall equation for the competition experiment is as follows:

$$
\mathrm{Cu}(\mathrm{I}) \cdot \mathrm{BCS}_{2}+2 \cdot(4) \rightleftharpoons \mathrm{Cu}(\mathrm{I}) \cdot \mathrm{BCS} \cdot 4+\mathrm{BCS}+\mathbf{4} \rightleftharpoons \mathrm{Cu}(\mathrm{I}) \cdot(4)_{2}+2 \cdot \mathrm{BCS}
$$

The titration results above indicate nearly complete consumption of $\mathrm{Cu}(\mathrm{I}) \cdot \mathrm{BCS}_{2}$ after addition of $\approx 1$ equiv. of diisonitrile 4 . These data are consistent with formation of the intermediate mixed complex $\mathrm{Cu}(\mathrm{I}) \cdot \mathrm{BCS} \cdot 4$ and with that complex having little or no interfering absorbance at 483 $\mathrm{nm}$. Beyond that, because the titration was essentially stoichiometric, we were unable to establish a competitive equilibrium between BCS and diisonitrile acetate $\mathbf{4}$ for coordination of $\mathrm{Cu}(\mathrm{I})$ and, hence, were unable to calculate association constants for diisonitrile acetate 4 with $\mathrm{Cu}(\mathrm{I})$. 


\section{Competitive titration experiments of $\mathrm{Cu}(\mathrm{BCS})_{2}$ with $\mathrm{SF} 2768$ and 5-epi-SF2768}

The analogous competitive titration experiments of $\mathrm{Cu}(\mathrm{BCS})_{2}$ were also carried out with SF2768 $((\mathbf{2 L}, \mathbf{5 S})-\mathbf{1})$ and 5-epi-SF2768 ((2L,5R)-1). Similar results were obtained, showing stoichiometric consumption of $\mathrm{Cu}(\mathrm{BCS})_{2}$ over addition of the first equivalent of the diisonitrile.
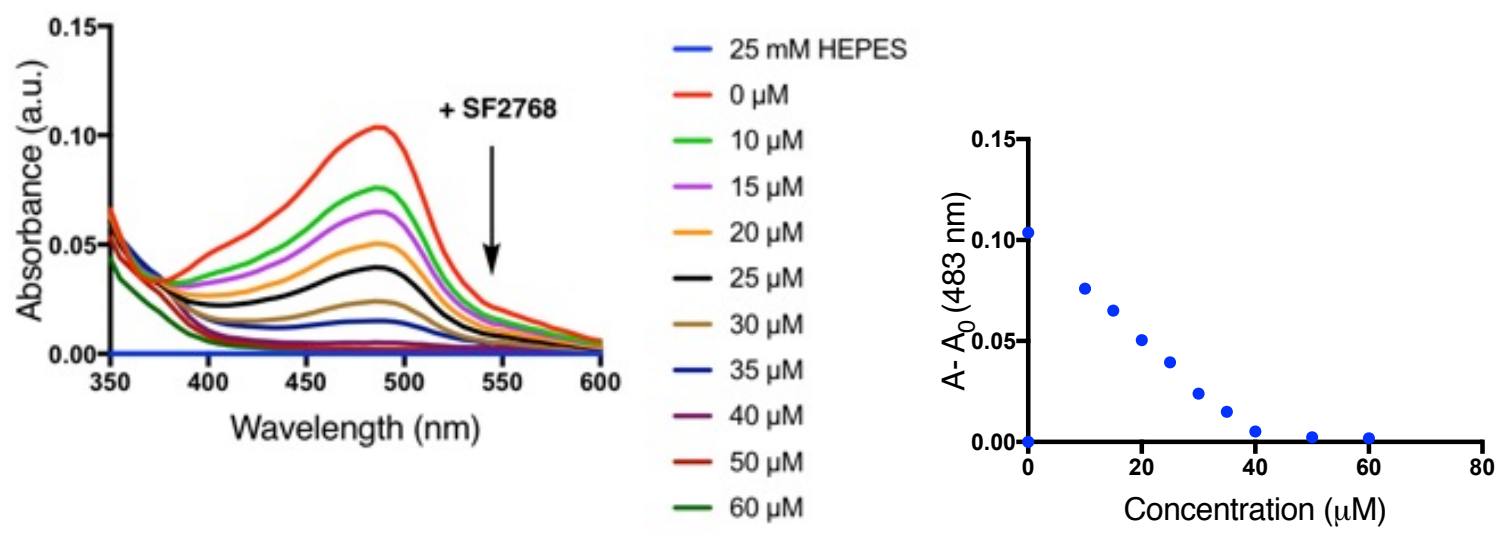

Figure S18. UV-Vis titration of $\mathrm{Cu}(\mathrm{BCS})_{2}$ with various equivalents of SF2768 ((2L,5S)-1).
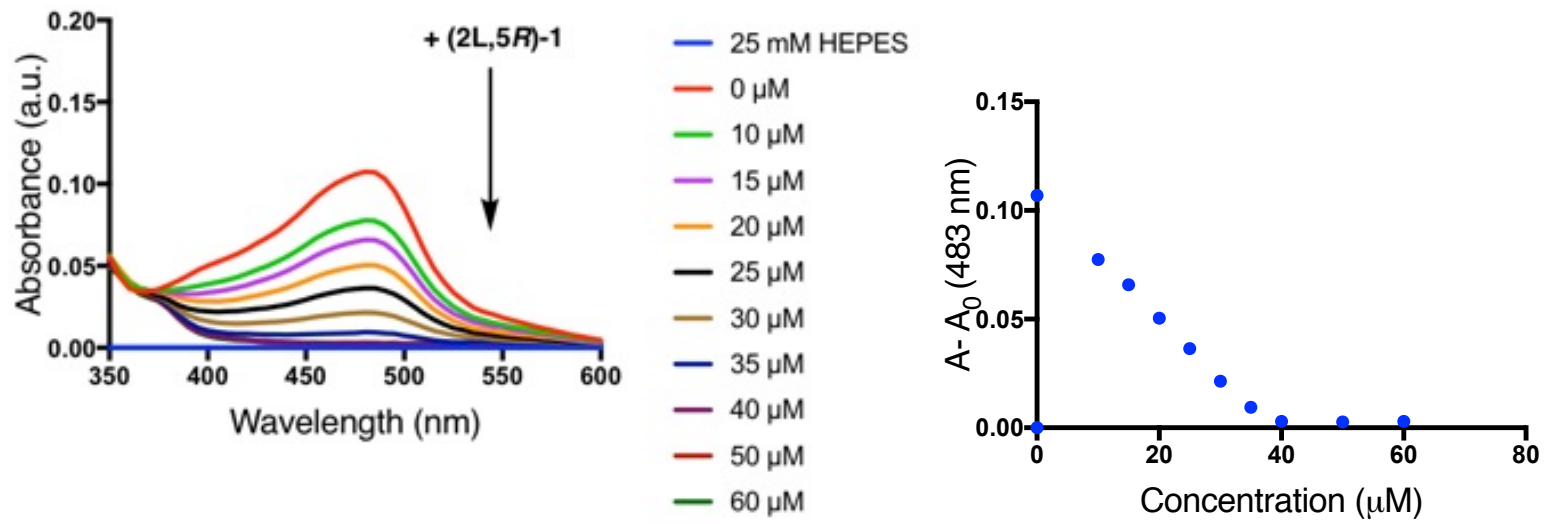

Figure $\mathrm{S} 19 . \mathrm{UV}-\mathrm{Vis}$ titration of $\mathrm{Cu}(\mathrm{BCS})_{2}$ with various equivalents of 5-epi-SF2768 $((2 \mathrm{~L}, 5 R)-1)$. 


\section{Competitive titration experiments of $\mathrm{Cu}(4)_{2}(\mathrm{~S} 8)$ with $\mathrm{BCS}$}

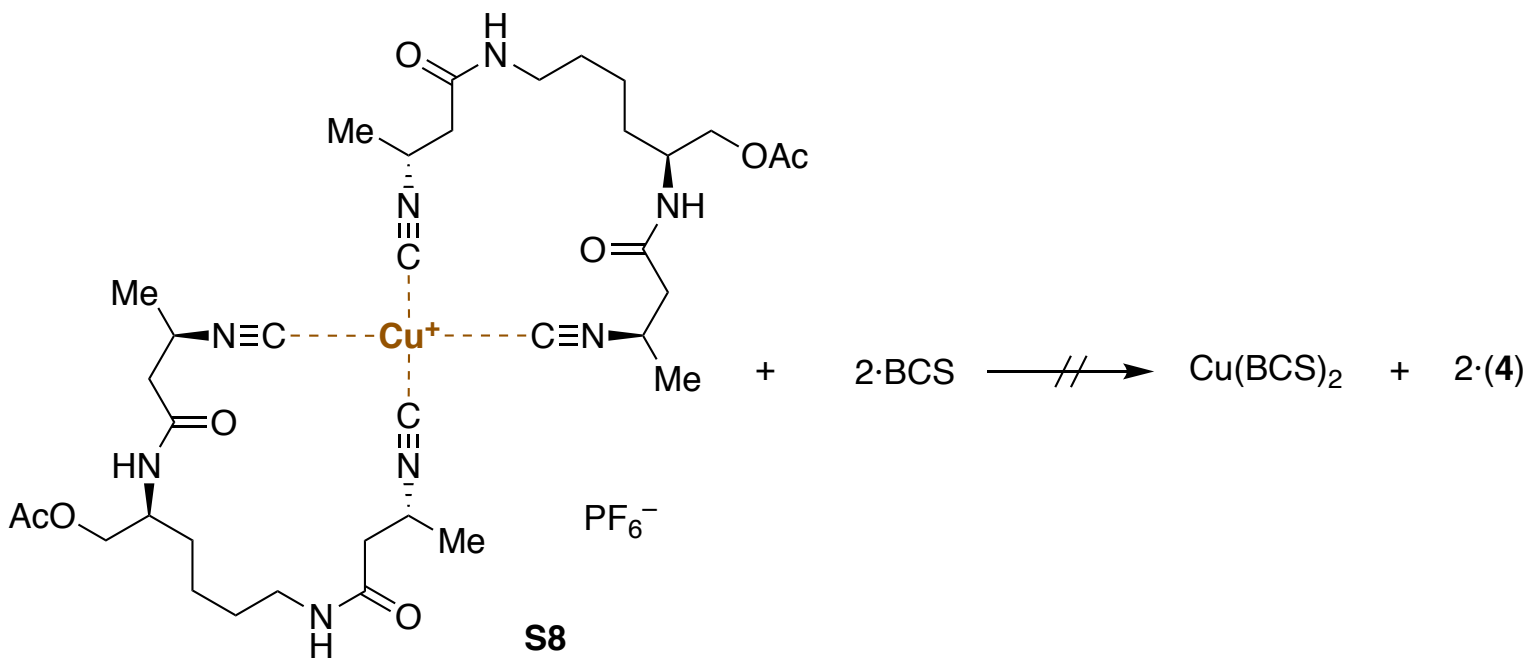

A solution of $25 \mathrm{mM}$ HEPES buffer $\left(0.1 \mathrm{M} \mathrm{NaCl}, \mathrm{pH} 7.2, \mathrm{MeCN}: \mathrm{H}_{2} \mathrm{O} v / \mathrm{v}=1: 10\right)$ was prepared by diluting commercial 1.0 M HEPES buffer (Corning) with Milli-Q water and degassed MeCN. The diluted HEPES buffer was then degassed and deoxygenated using a sonicator and Ar.

Stock solutions of $1 \mathrm{mM}, 2 \mathrm{mM}, 3 \mathrm{mM}, 4 \mathrm{mM}, 5 \mathrm{mM}, 6 \mathrm{mM}, 8 \mathrm{mM}, 10 \mathrm{mM}, 12 \mathrm{mM}, 15 \mathrm{mM}$, $20 \mathrm{mM}, 40 \mathrm{mM}, 100 \mathrm{mM}, 200 \mathrm{mM}$, and $400 \mathrm{mM}$ BCS were prepared in $25 \mathrm{mM}$ HEPES buffer. A $1 \mathrm{mM}$ stock solution of the $\mathrm{Cu}(\mathrm{I})$ complex with diisonitrile 4 (S8) was prepared in degassed, anhydrous DMSO. Aliquots of $1.2 \mu \mathrm{L}$ of each stock solution of BCS were added to aliquots of $4.8 \mu \mathrm{L}$ of the copper complex of diisonitrile 4 (S8) and diluted to $120 \mu \mathrm{L}$ with $25 \mathrm{mM}$ HEPES buffer. Samples of $100 \mu \mathrm{L}$ of each final solution were analyzed by UV-Vis. ${ }^{20}$ No competitive binding of BCS was observed, even with up to 100 equiv $(4000 \mu \mathrm{M})$.
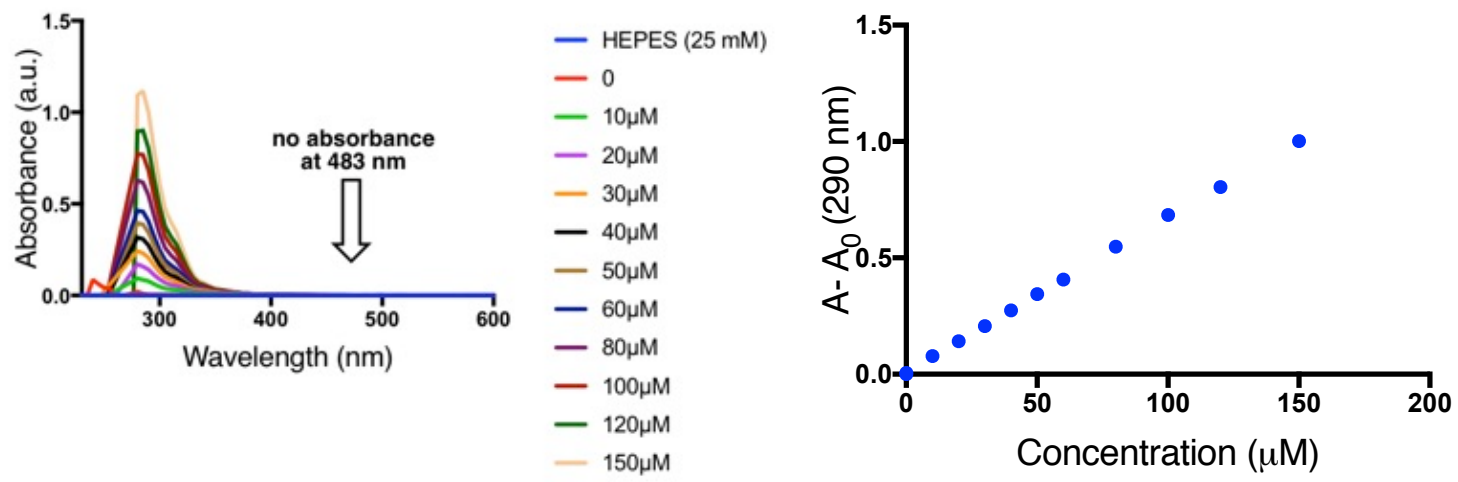

Figure S20. UV-Vis titration of the $\mathrm{Cu}(\mathrm{l})$ complex of diisonitrile 4 (S8) (40 $\mu \mathrm{M}$ ) with various equivalents of BCS. Increasing absorbance at $290 \mathrm{~nm}$ is observed, corresponding to free BCS, while no absorbance at $483 \mathrm{~nm}$ is observed, indicating no formation of $\mathrm{Cu}(\mathrm{BCS})_{2}$. 

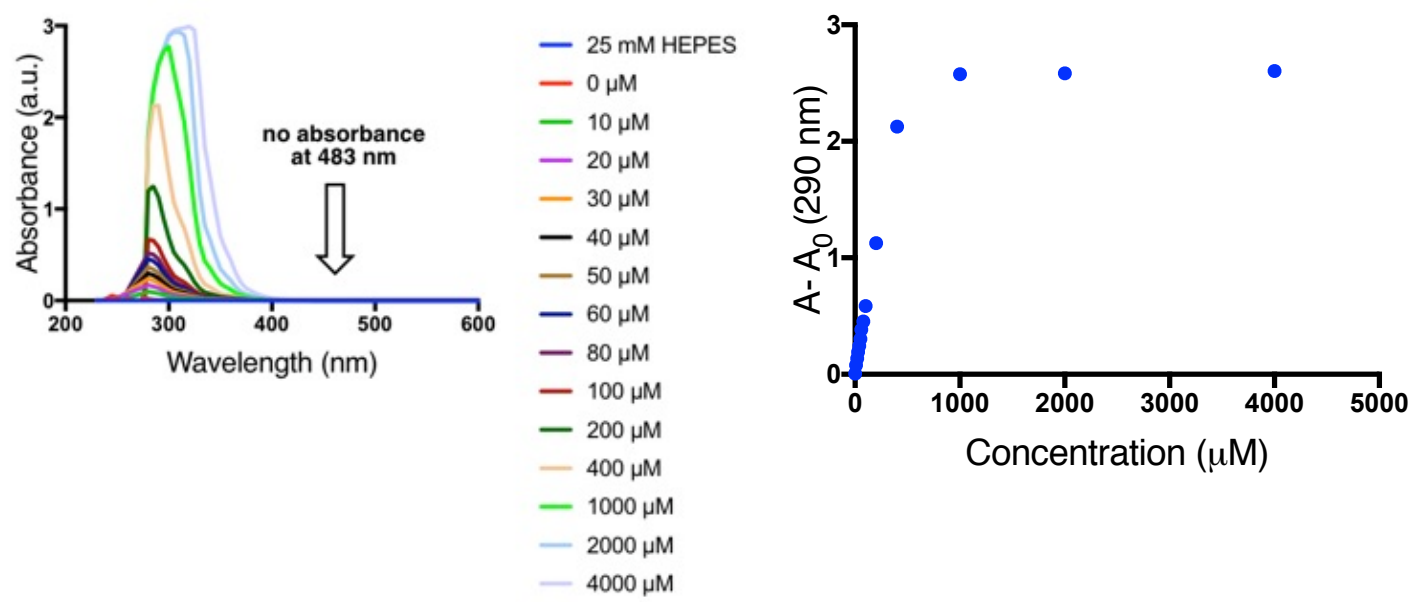

Figure S21. UV-Vis titration of the Cu(I) complex of diisonitrile 4 (S8 (40 $\mu \mathrm{M})$ with large excesses of BCS. Increasing absorbance at $290 \mathrm{~nm}$ is observed, corresponding to free BCS, until saturation of the signal $(\approx 1000 \mu \mathrm{M})$, while no absorbance at $483 \mathrm{~nm}$ is observed, indicating no formation of $\mathrm{Cu}(\mathrm{BCS})_{2}$ with up to 100 -fold excess of BCS relative to the $\mathrm{Cu}(\mathrm{I})$ complex of diisonitrile 4 (S8) . 


\section{Competitive titration experiments of $\mathrm{Cu}(\mathrm{SF2768})_{2}$ (S9) and $\mathrm{Cu}(5-e p i-S F 2768)_{2}$ (S10) with $B C S$}

The analogous competitive titration experiments were also carried out with the $\mathrm{Cu}(\mathrm{I})$ complex of SF2768 (S9) as well as the $\mathrm{Cu}(\mathrm{I})$ complex of 5-epi-SF2768 (S10). Similar results were obtained, showing no competitive $\mathrm{Cu}(\mathrm{I})$ binding of BCS, even with up 100 equiv $(4000 \mu \mathrm{M})$.
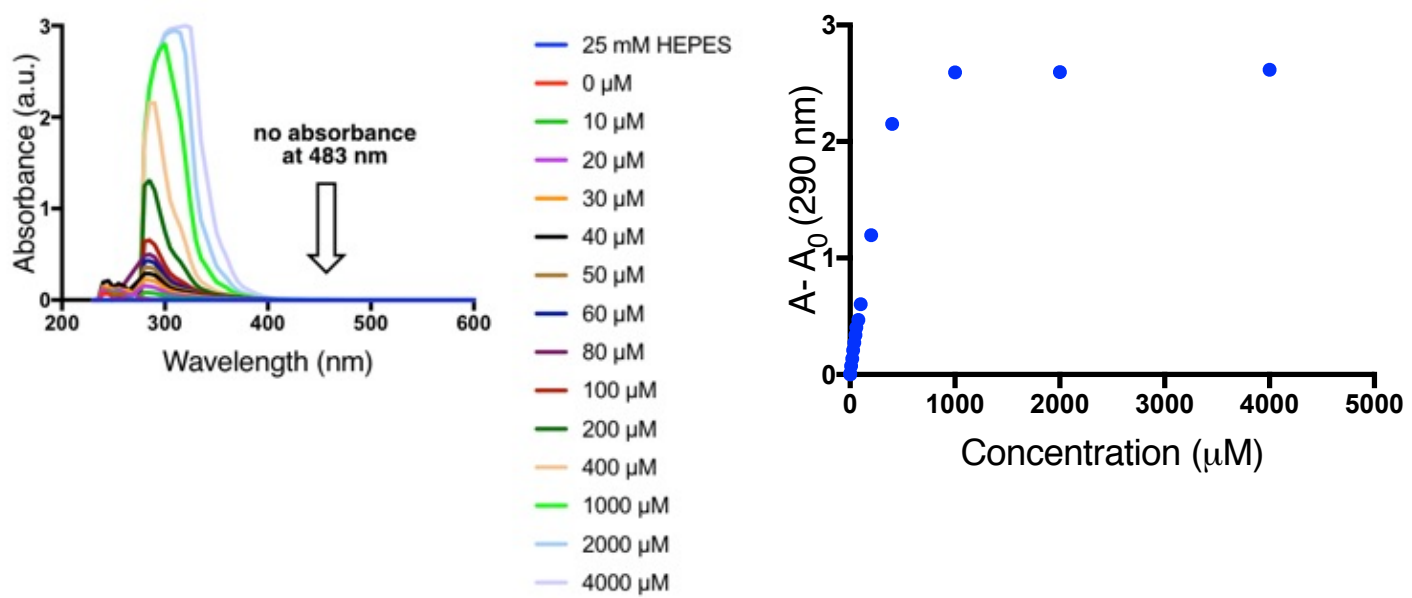

Figure S22. UV-Vis titration of the Cu(I) complex of SF2768 (S9) (40 $\mu \mathrm{M})$ with large excesses of BCS. Increasing absorbance at $290 \mathrm{~nm}$ is observed, corresponding to free BCS, until saturation of the signal $(\approx 1000 \mu \mathrm{M})$, while no absorbance at $483 \mathrm{~nm}$ is observed, indicating no formation of $\mathrm{Cu}(\mathrm{BCS})_{2}$ with up to 100 -fold excess of BCS relative to the $\mathrm{Cu}(\mathrm{I})$ complex of SF2768 (S9).
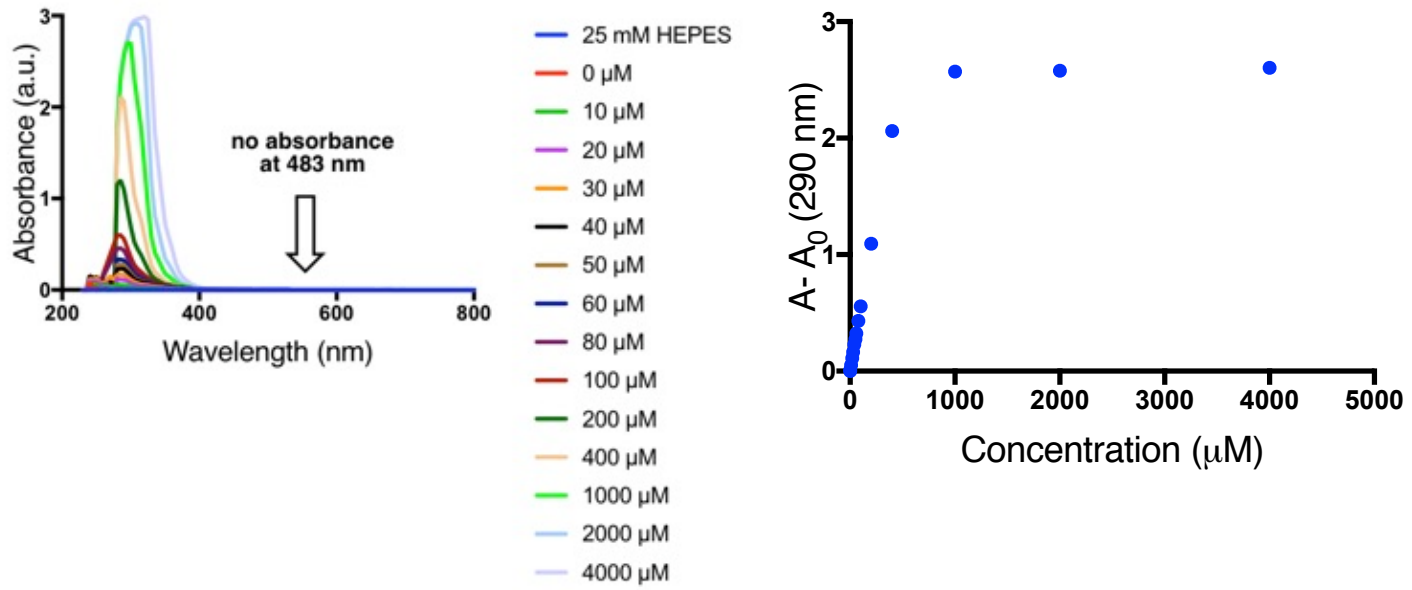

Figure S23. UV-Vis titration of the $\mathrm{Cu}(\mathrm{I})$ complex of 5-epi-SF2768 (S10) (40 $\mu \mathrm{M})$ with large excesses of BCS. Increasing absorbance at $290 \mathrm{~nm}$ is observed, corresponding to free BCS, until saturation of the signal $(\approx 1000 \mu \mathrm{M})$, while no absorbance at $483 \mathrm{~nm}$ is observed, indicating no formation of $\mathrm{Cu}(\mathrm{BCS})_{2}$ with up to 100 -fold excess of BCS relative to the $\mathrm{Cu}(\mathrm{I})$ complex of 5-epi-SF2768 (S10). 
K. AtTempted Formation of Zn(II) Complex of Diisonitrile Acetate 4

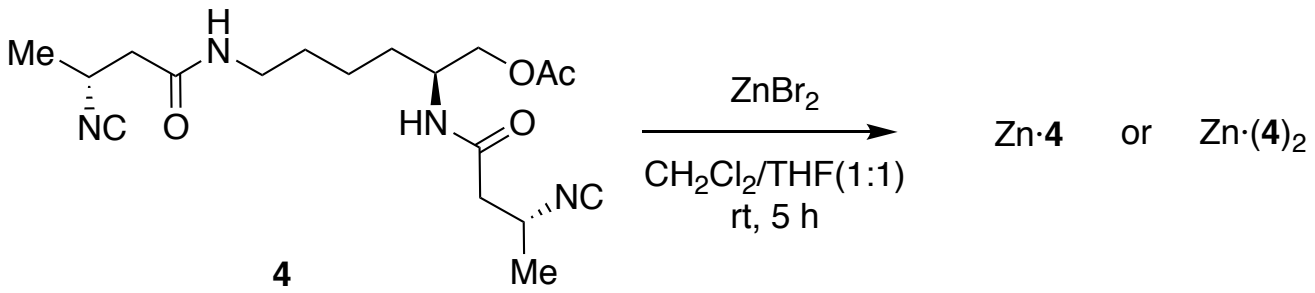

In a flame-dried $13 \times 100 \mathrm{~mm}$ test tube, in the glovebox, were placed $(S)$-2,6-bis $((R)-3$-isocyanobutanamido)hexyl acetate 4 (7.28 mg, $0.0200 \mathrm{mmol}, 1.0$ equiv) and $\mathrm{ZnBr}_{2}$ (4.5 $\mathrm{mg}, 0.0200$ mmol, 1.0 equiv). The test tube was sealed with a septum and removed from the glovebox, followed by addition of $\mathrm{CH}_{2} \mathrm{Cl}_{2}(0.3 \mathrm{~mL})$ and THF $(0.3 \mathrm{~mL})$. The mixture was stirred at $\mathrm{rt}$ for $5 \mathrm{~h}$. Analysis by direct injection ESI-MS led to detection of the free diisonitrile $4(\mathrm{~m} / \mathrm{z} 365.4$, $[\mathrm{M}+\mathrm{H}]^{+}, 100 \%$ rel int) and 1:1 complex with zinc $(\mathrm{Zn} \cdot 4)\left(215.4,[\mathrm{M}+\mathrm{Zn}]^{2+} / 2,72 \%\right)$. However, the 2:1 complex $\left(\mathrm{Zn} \cdot(\mathbf{4})_{2}\right)$ was not observed $\left(396.2,[2 \mathrm{M}+\mathrm{Zn}]^{2+} / 2\right)$. In the ${ }^{1} \mathrm{H}-\mathrm{NMR}$ spectrum of the mixture, only peaks for the free diisonitrile 4 could be readily assigned, with considerable peak broadening. Taken together, these results suggest that diisonitrile $\mathbf{4}$ coordinates to $\mathrm{Zn}$ (II) much more weakly than to $\mathrm{Cu}(\mathrm{I})$ ( $c f$. SECTIONS $\mathrm{H}$ and I above), and as a 1:1 complex rather than a 2:1 complex.

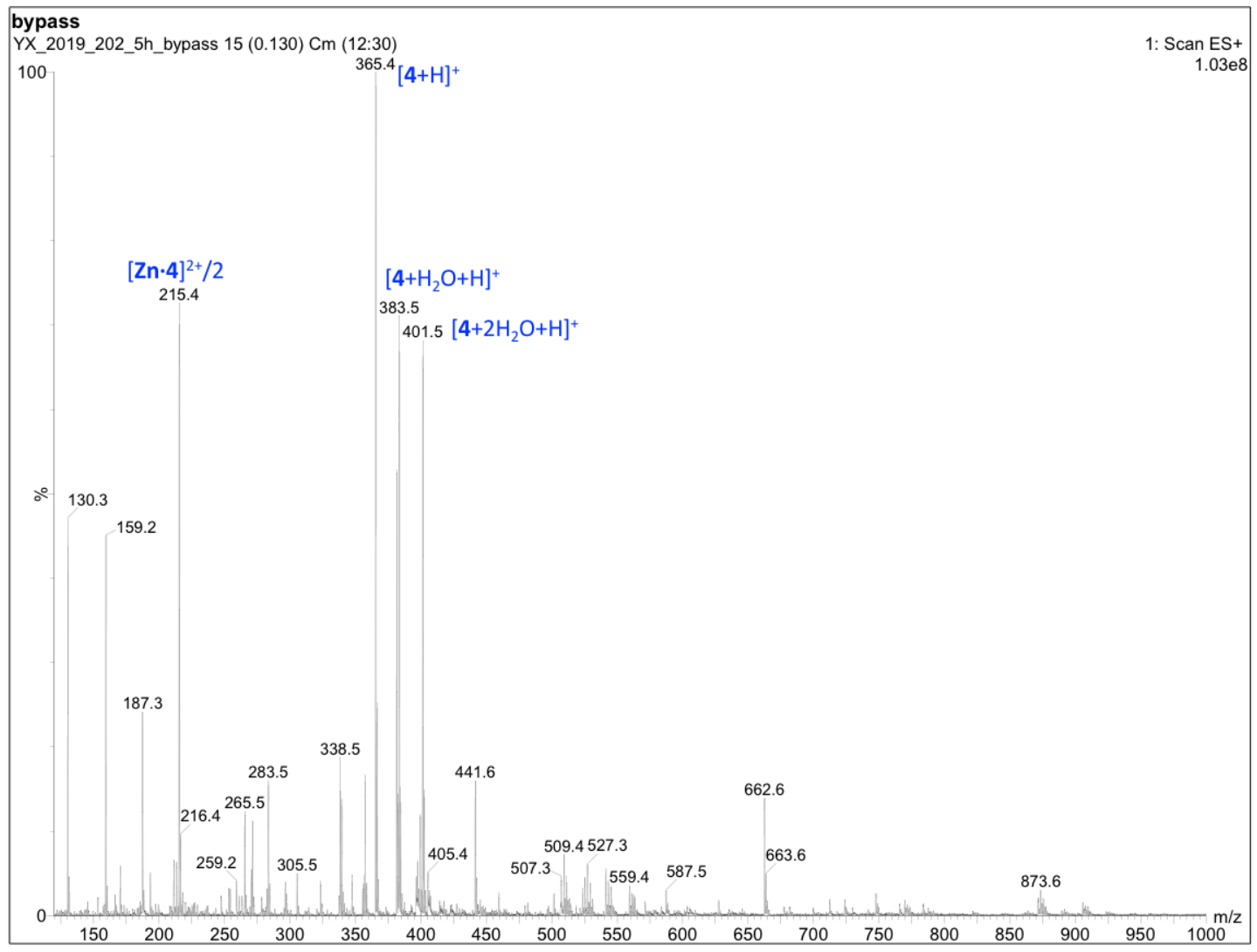

Figure S24. Direct-injection ESI-MS spectrum of reaction mixture from attempted formation of Zn(II) complex with diisonitrile acetate 4. 


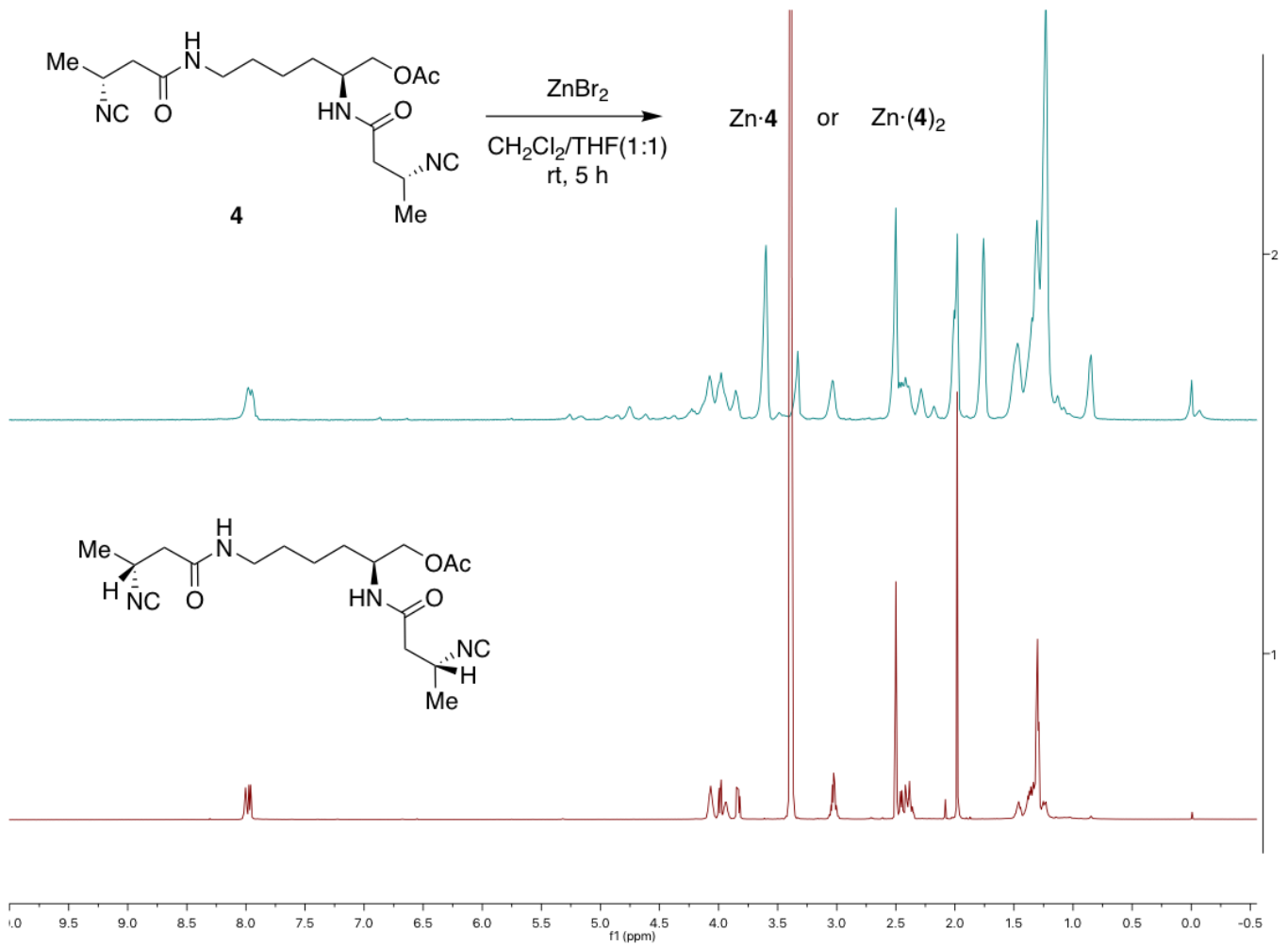

Figure S25. Stacked ${ }^{1} \mathrm{H}-\mathrm{NMR}$ of diisonitrile acetate 4 and the mixture resulting from reaction with with $\mathrm{ZnBr}_{2}$ (1 equiv). 


\section{L. ${ }^{1}$ H-NMR AND ${ }^{13}$ C-NMR SPECTRA}

1. SYNTHESIS OF NHS ESTER $5 \quad$ S49

a. (R)-3-Formamidobutanoic acid (11) $\quad$ S49

b. (R)-3-Formamidobutanoate NHS ester (12) S50

c. (R)-3-Isocyanobutanoate NHS ester (5) S51

2. SYNTHESIS OF AZIDOALCOHOLS $8 \quad$ S52

a. $N$-Boc-oxazinone azidoalcohol $(2 \mathrm{~L}, 5 S)-8 \quad$ S52

b. $N$-Boc-oxazinone azidoalcohol $(\mathbf{2} \mathbf{L}, \mathbf{5 R})-8 \quad$ S53

3. SYNTHESIS OF SF2768 (2L,5S-1) AND 5-EPI-SF2768 (2L,5R-1) S54

a. Oxazinone azidoalcohol $(2 \mathrm{~L}, 5 S)-13 \quad$ S54

b. Oxazinone azidoalcohol $(2 \mathrm{~L}, \mathbf{5 R})-\mathbf{1 3} \quad \mathrm{S} 55$

c. $\delta$-Hydroxylysine methyl ester $(2 \mathrm{~L}, 5 S)-14 \quad$ S56

d. $\delta$-Hydroxylysine methyl ester $(2 \mathrm{~L}, \mathbf{5 R})-14 \quad$ S56

e. $\operatorname{Bis}(N$-Boc)- $\delta$-hydroxylysine methyl ester (2L,5S)-7 $\quad$ S57

f. $\quad \operatorname{Bis}(N$-Boc)- $\delta$-hydroxylysine methyl ester (2L,5 $\boldsymbol{R})-7 \quad$ S58

g. $\quad \operatorname{Bis}(N$-Boc)-diaminolactone (2L,5S)-15 (with COSY and NOESY) S59

h. $\operatorname{Bis}(N$-Boc)-diaminolactone (2L,5R)-15 (with COSY and NOESY) S61

i. $\operatorname{Bis}(N$-Boc)-diaminolactol (2L,5S)-16 (with COSY and HSQC) S63

j. $\operatorname{Bis}(N$-Boc)-diaminolactol (2L,5R)-16 (with COSY and HSQC) S65

k. SF2768, (2L,5S)-1 (with COSY, NOESY, HMBC, HSQC, authentic samples) S65

1. 5-epi-SF2768, (2L,5R)-1 (with COSY, NOESY, HMBC, HSQC) S72

4. Synthesis OF ACYCLIC DiISONITRILES 3 AND 4

$\begin{array}{ll}\text { a. } \operatorname{Bis}(N \text {-Boc)-lysinol S5 } & \mathrm{S} 75\end{array}$

$\begin{array}{ll}\text { b. } \operatorname{Bis}(N \text {-Boc)-lysinol acetate S6 } & \text { S76 }\end{array}$

$\begin{array}{ll}\text { c. Lysinol acetate } \cdot \text { bis(TFA) salt S7 } & \text { S77 }\end{array}$

d. Diisonitrile acetate 4 (with COSY, HSQC) $\quad$ S78

$\begin{array}{ll}\text { e. Diisonitrile alcohol } 3 & \text { S80 }\end{array}$

5. Synthesis of Cu(I) Complex of Dilsonitrile ACEtate 4

a. Diisonitrile acetate $(\mathbf{4})_{2} \cdot \mathrm{Cu}(\mathrm{I})$ complex $\mathbf{S 8}$ (with COSY, HSQC, HMBC) S81 


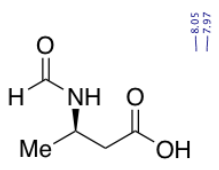

11
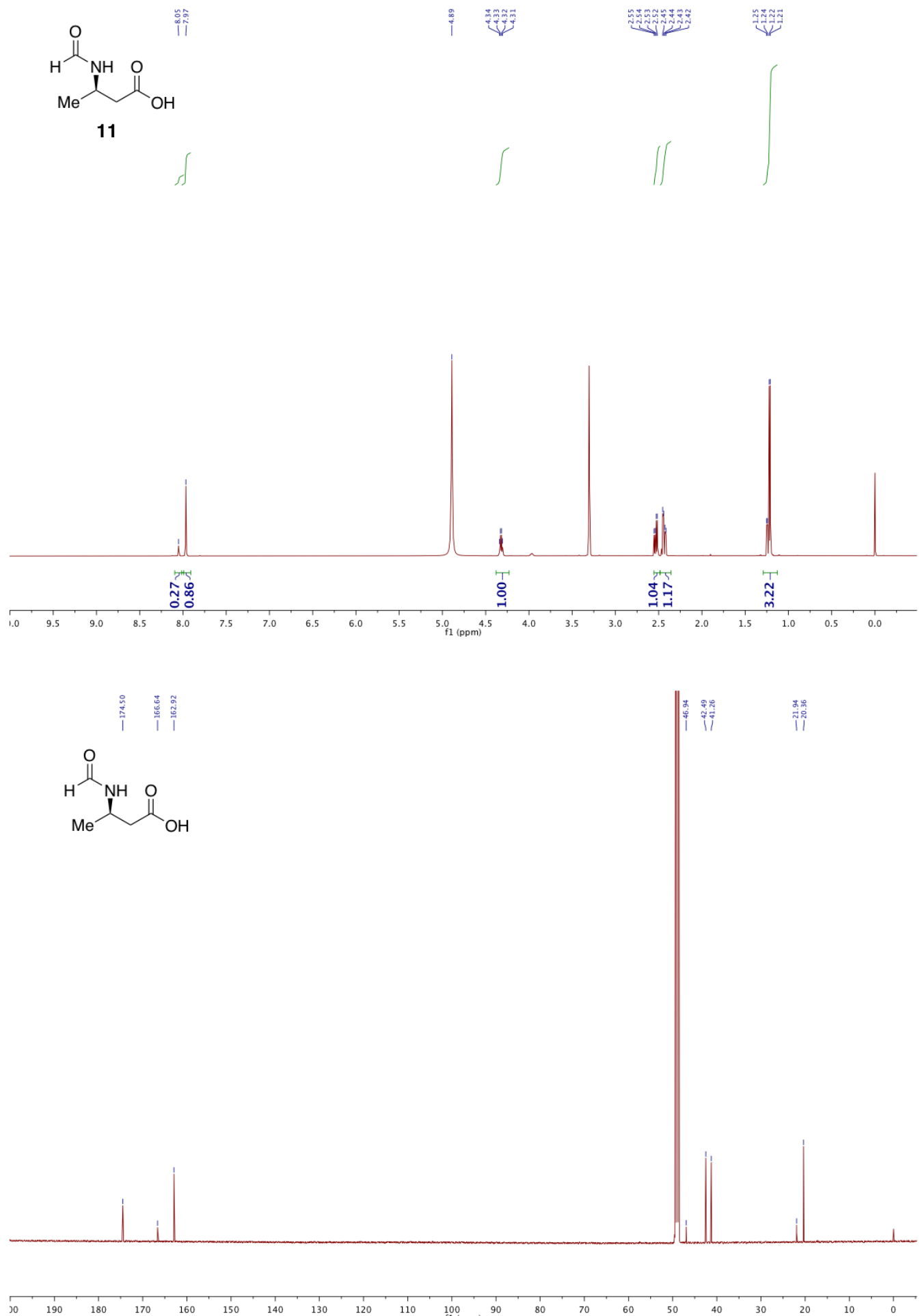


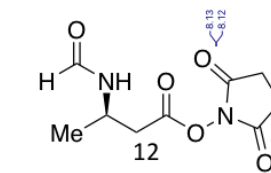

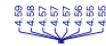

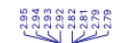
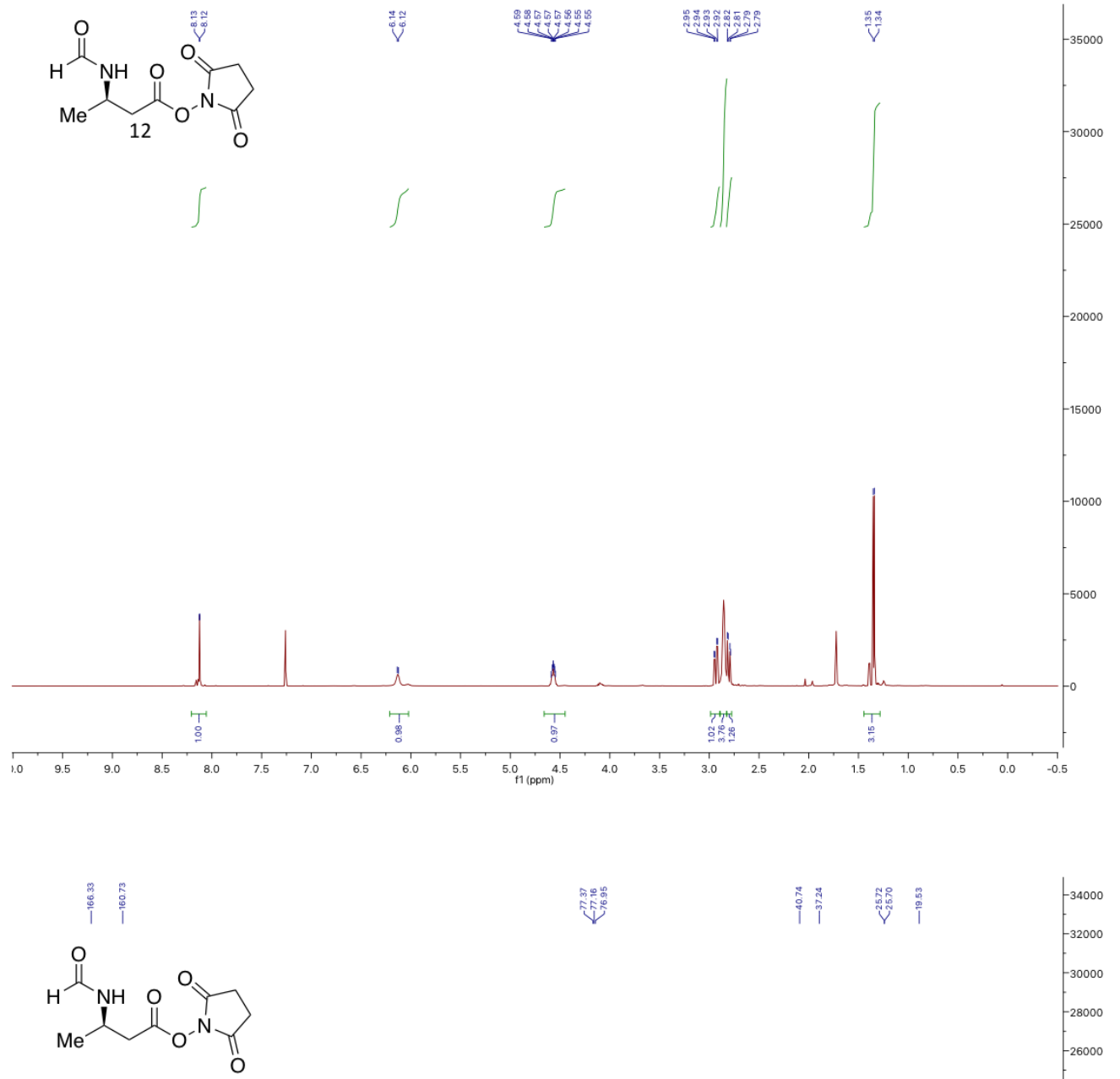

率昌
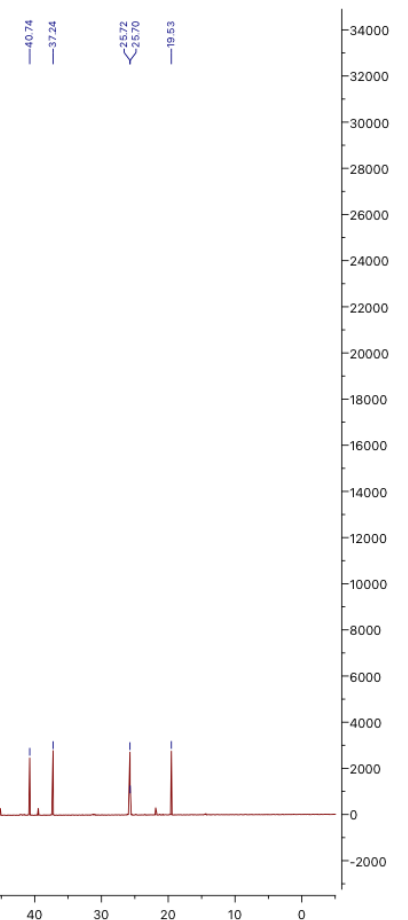

${ }_{30}$ 

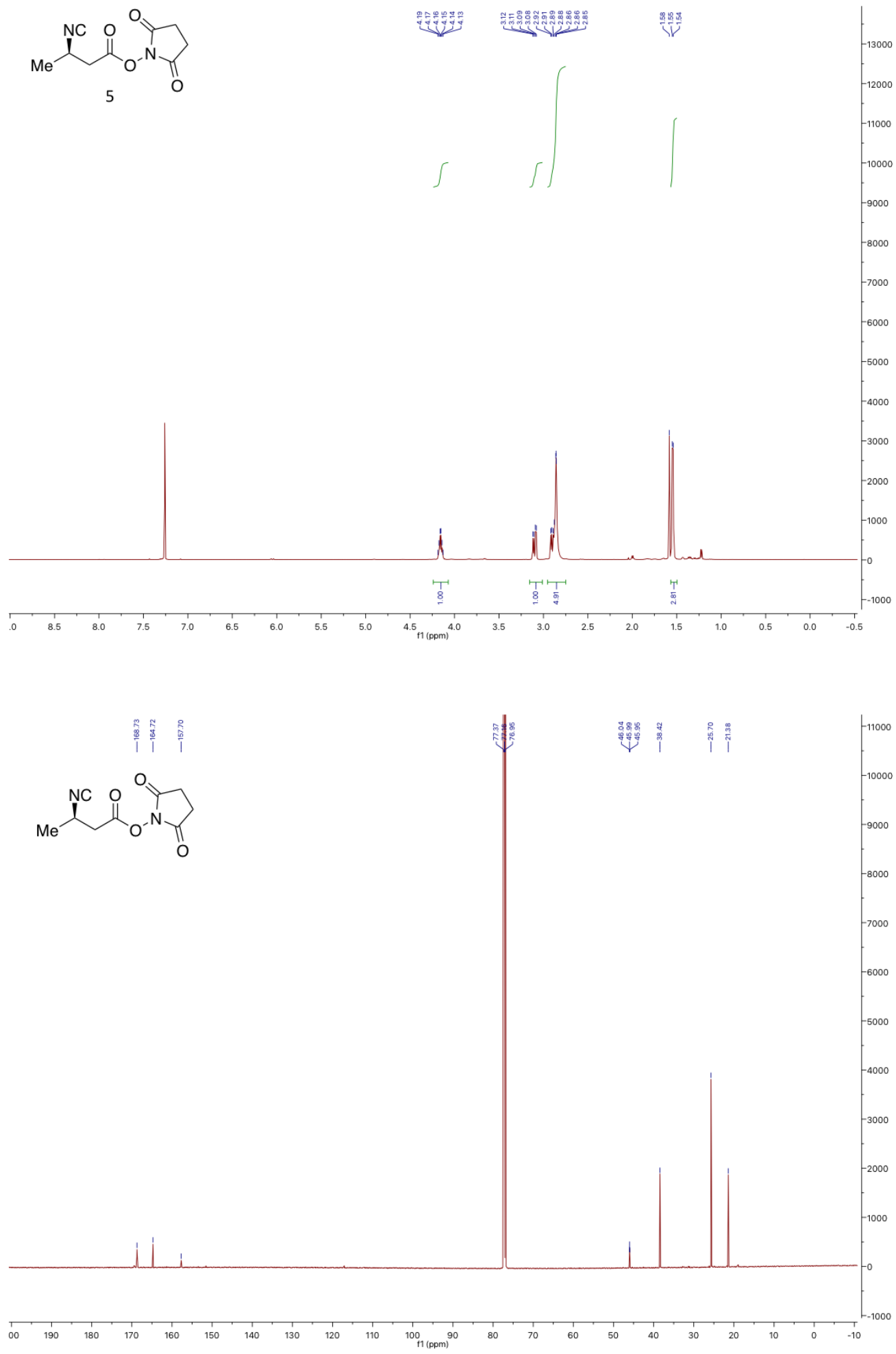

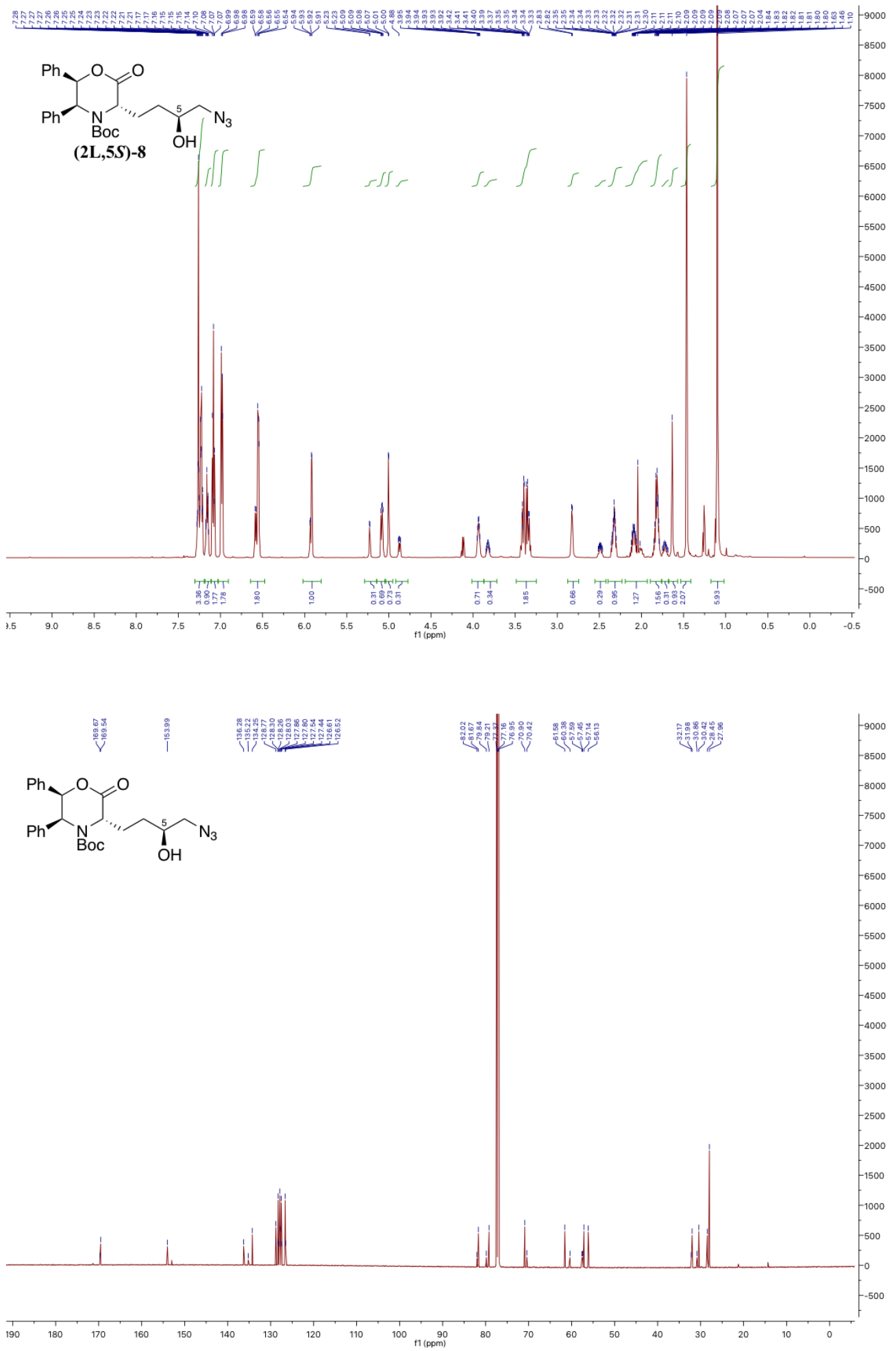

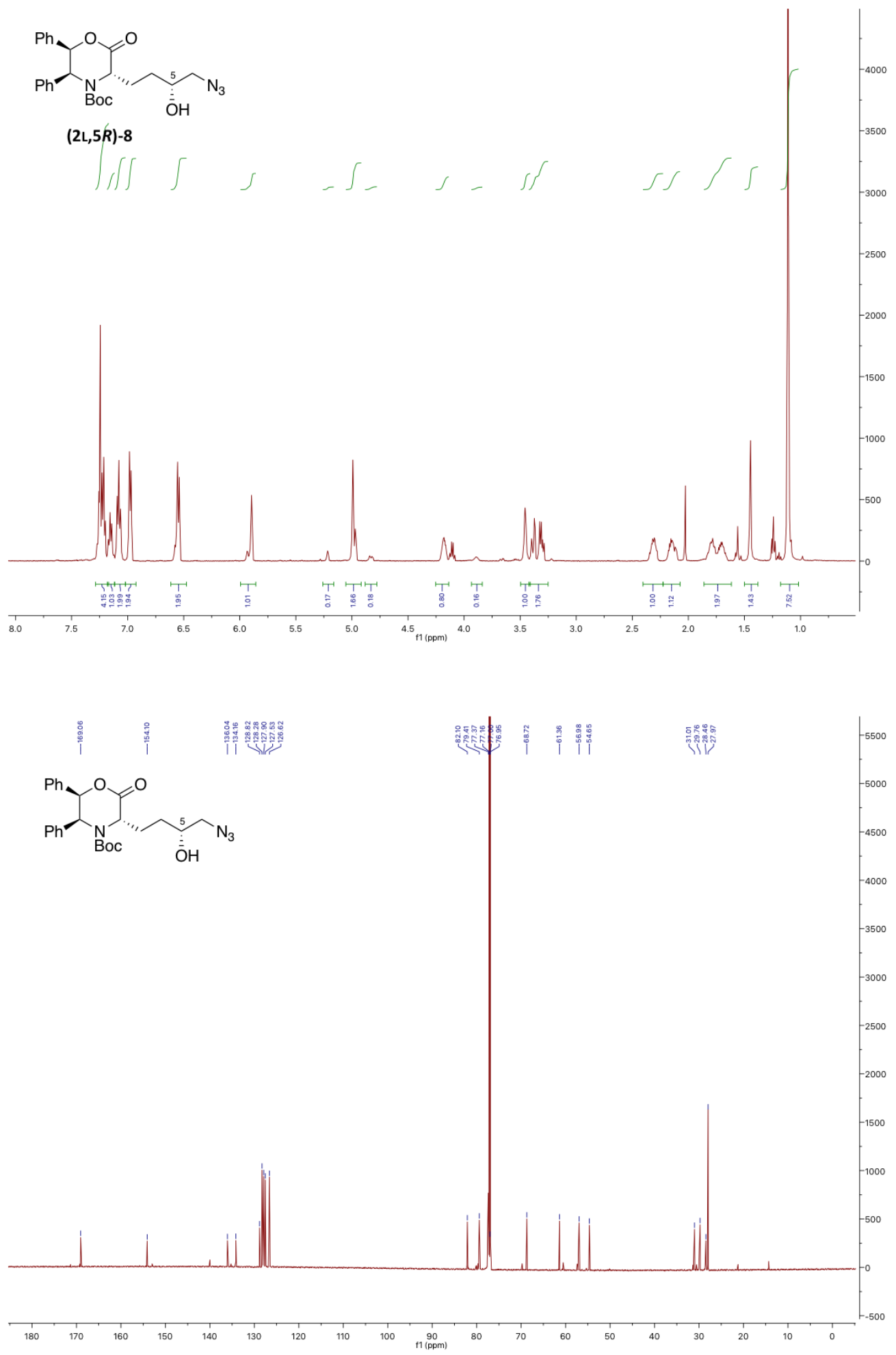

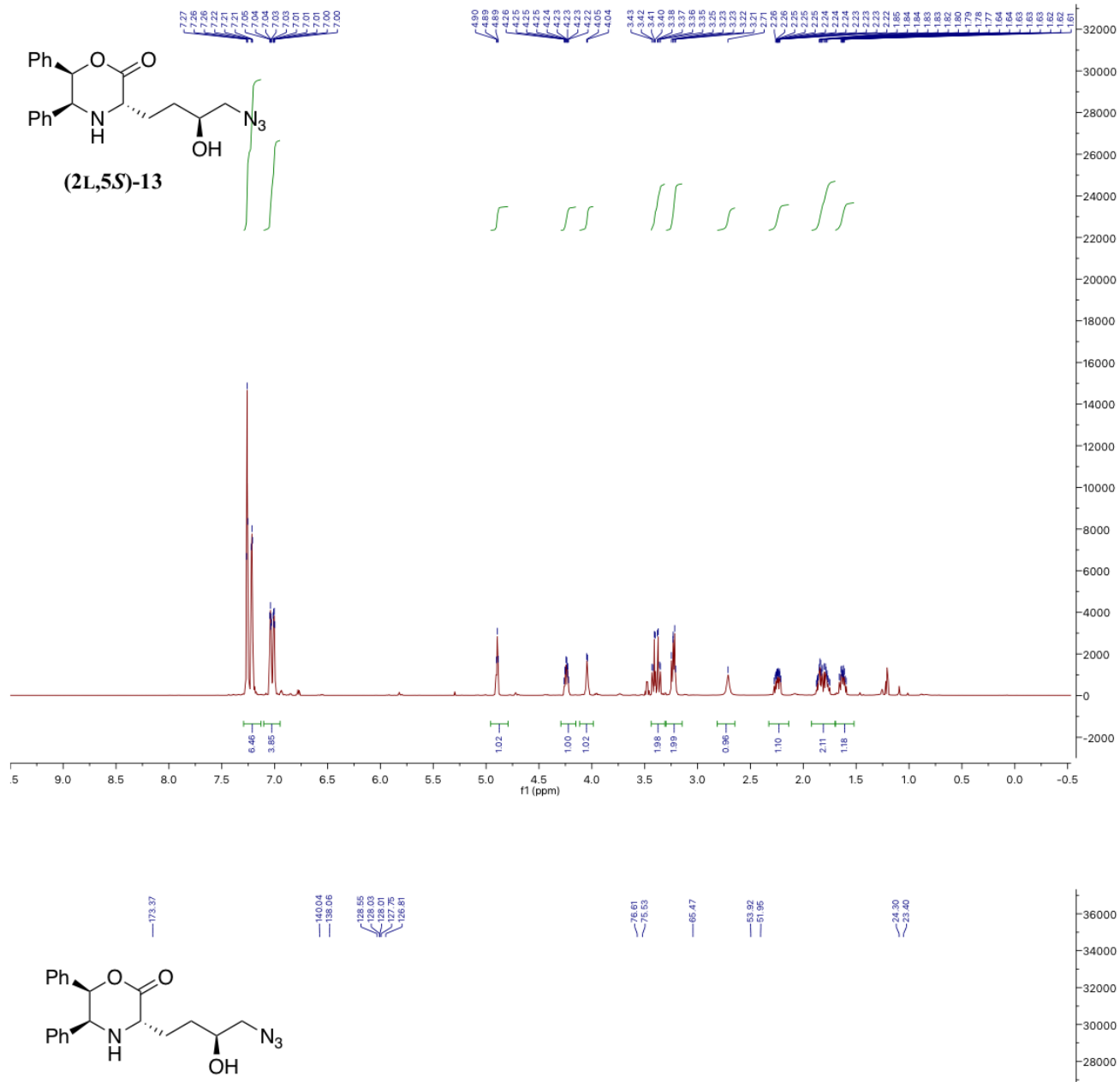

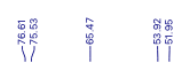

\section{政}

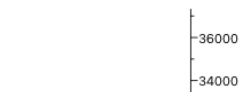



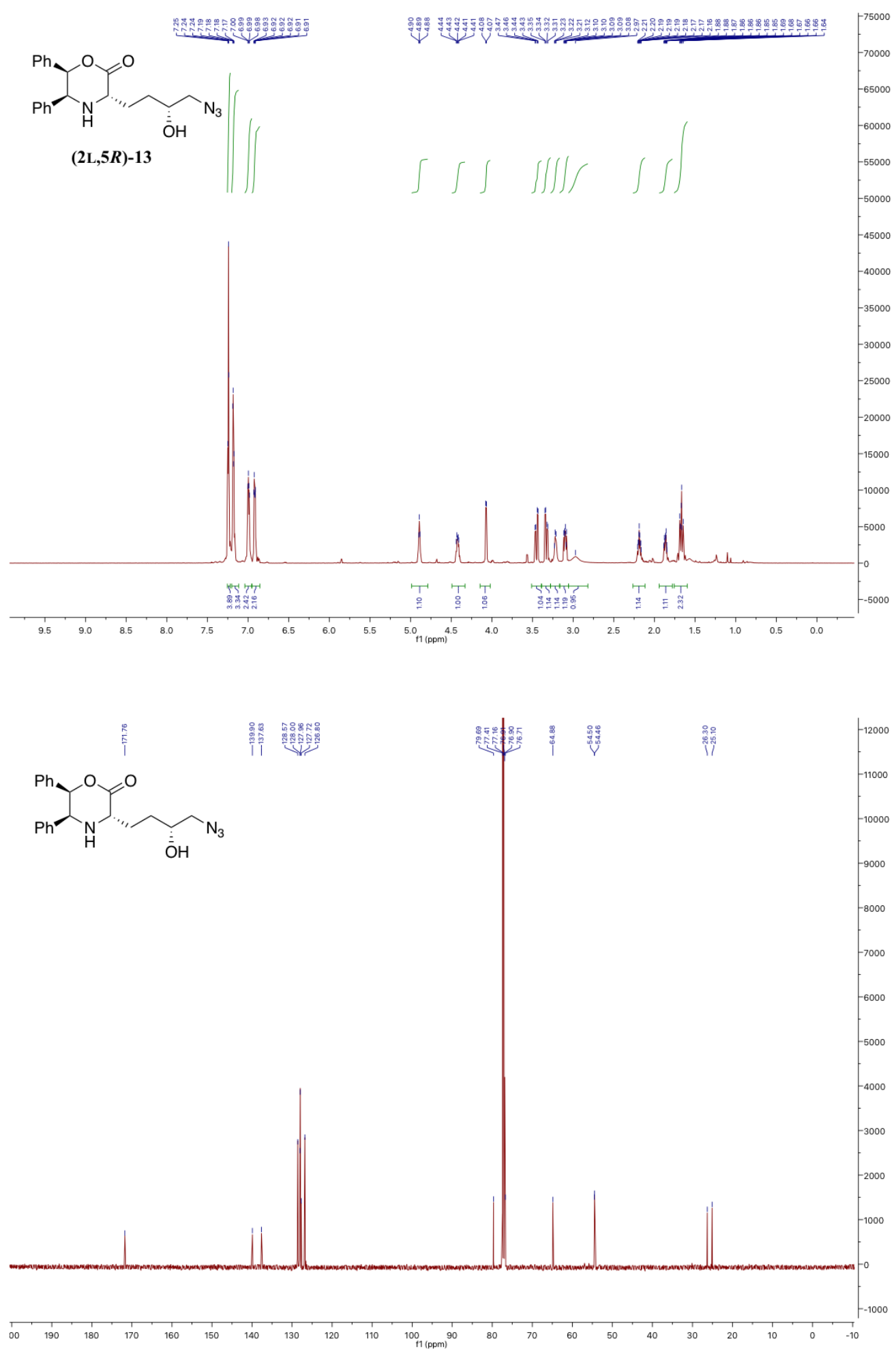


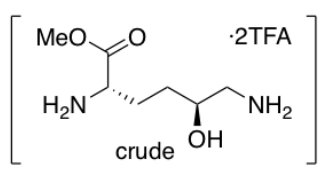

(2L,5S)-14
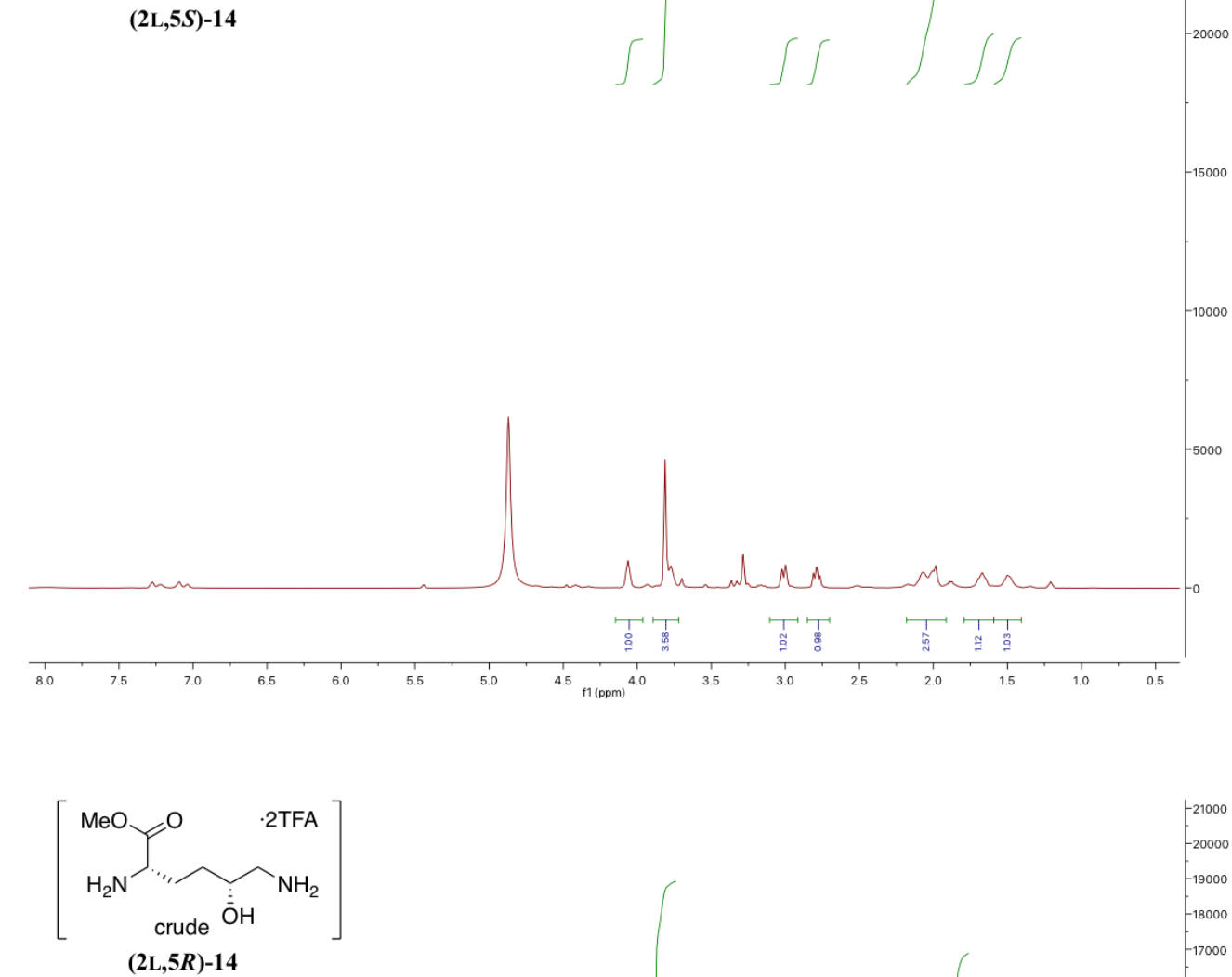

(2L,5R)-14

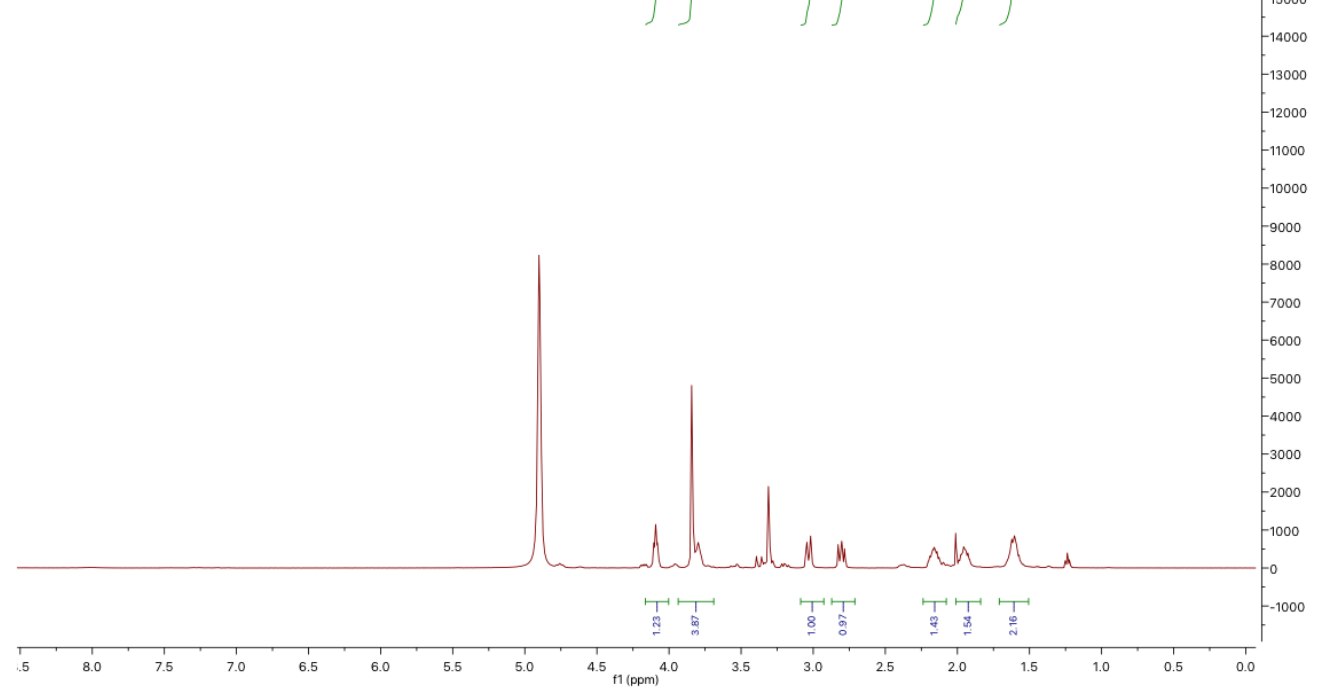



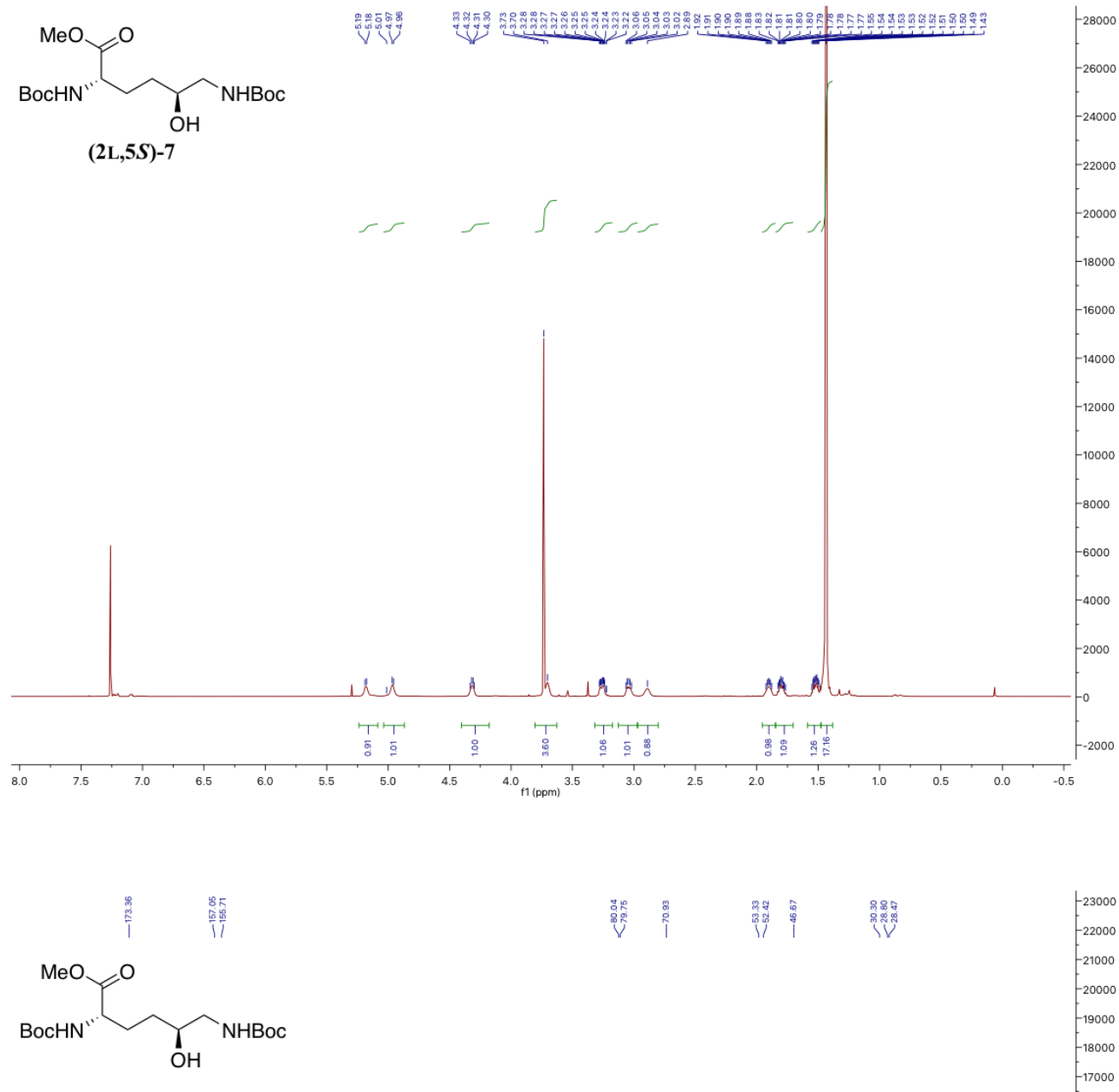

-23000
-22000
-21000
-20000
-19000
-18000
-17000
-16000

$-15000$

$-14000$

$-13000$

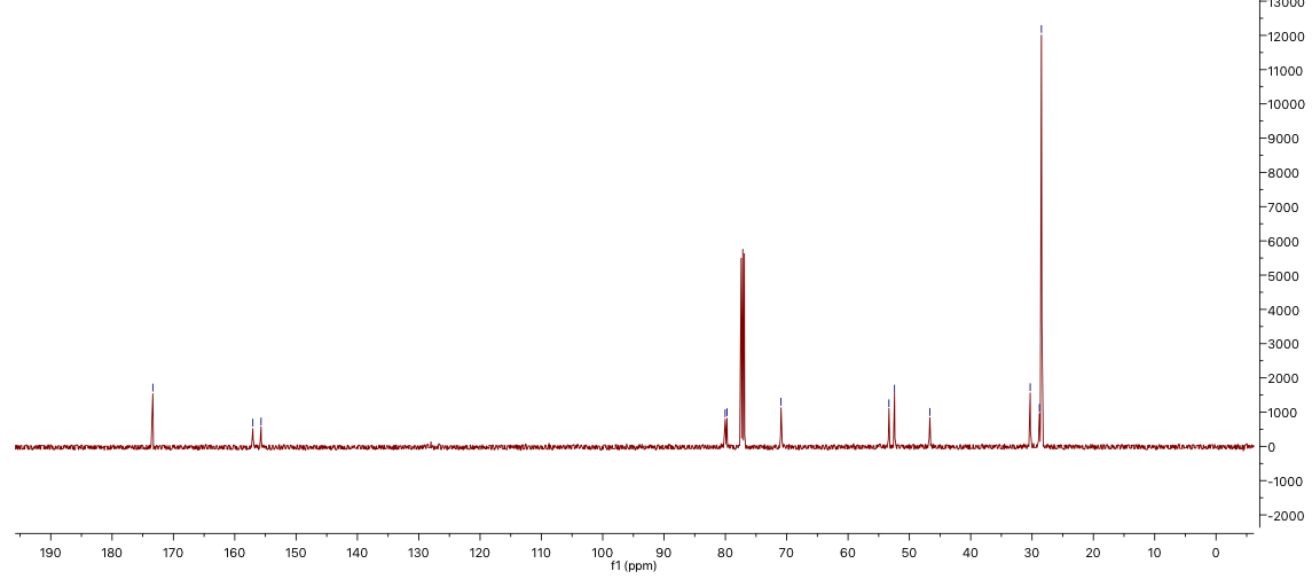



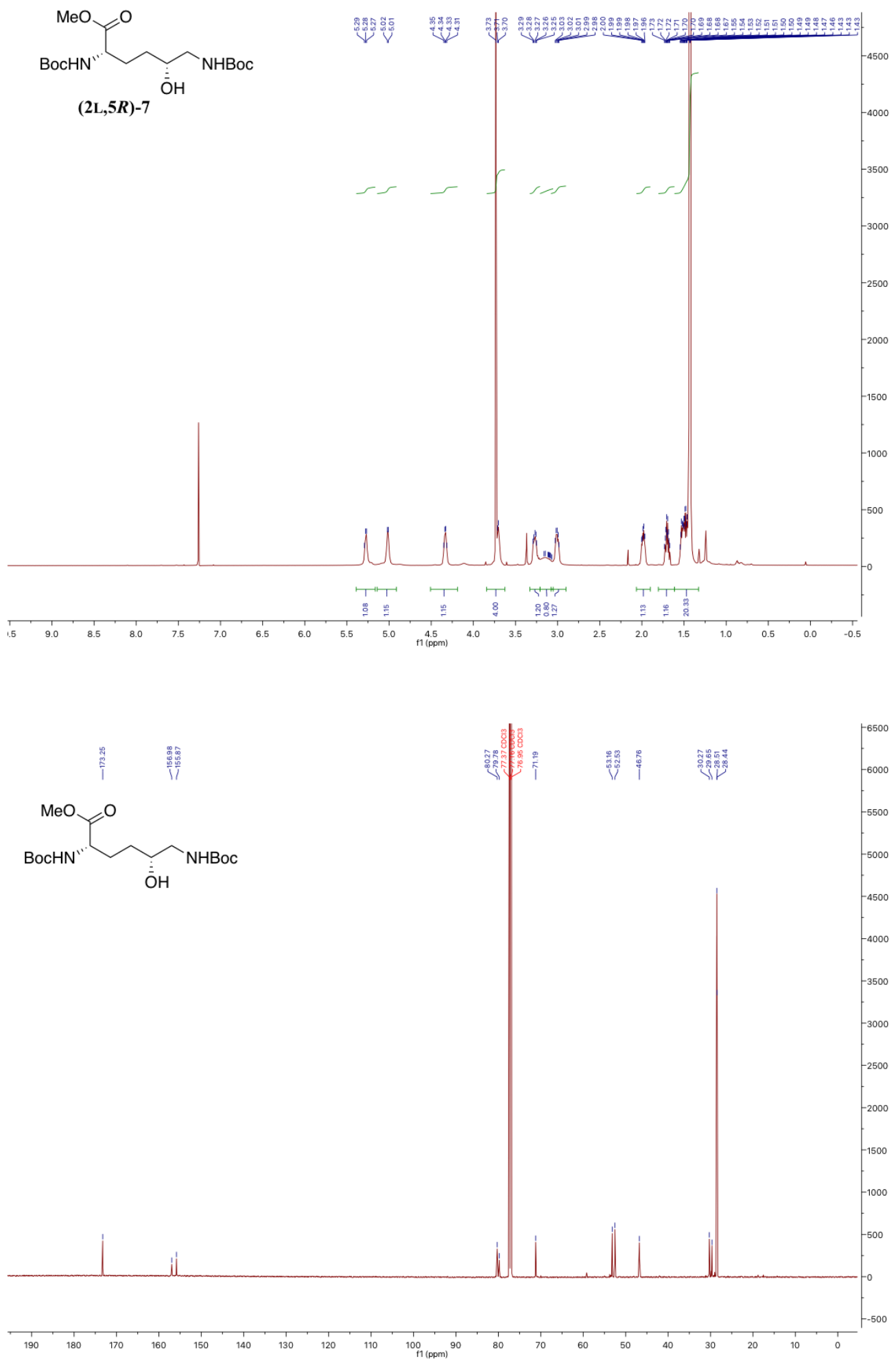


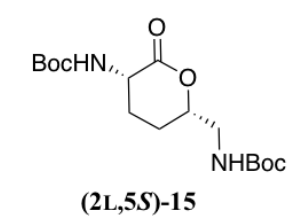

ทํ.
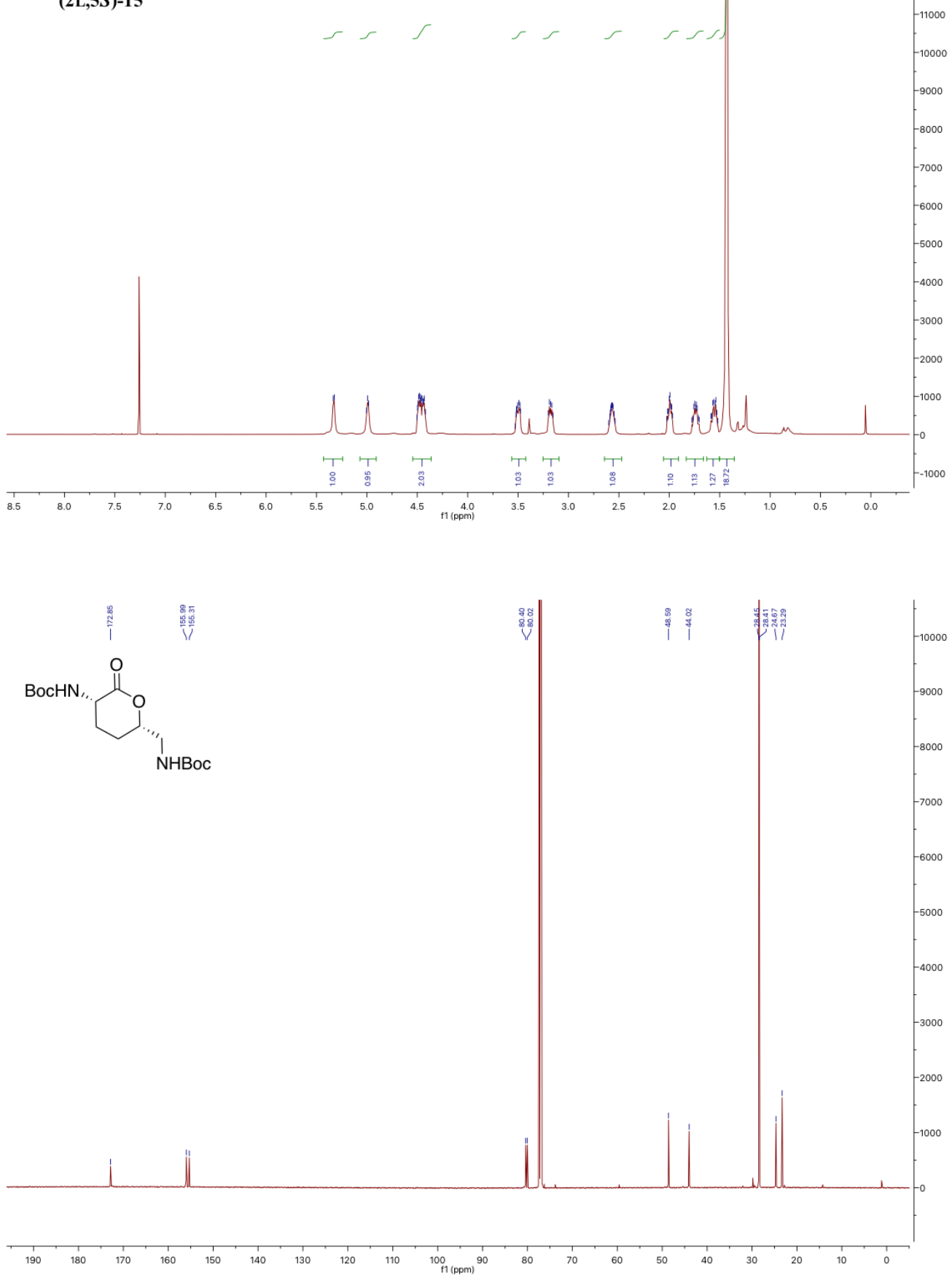

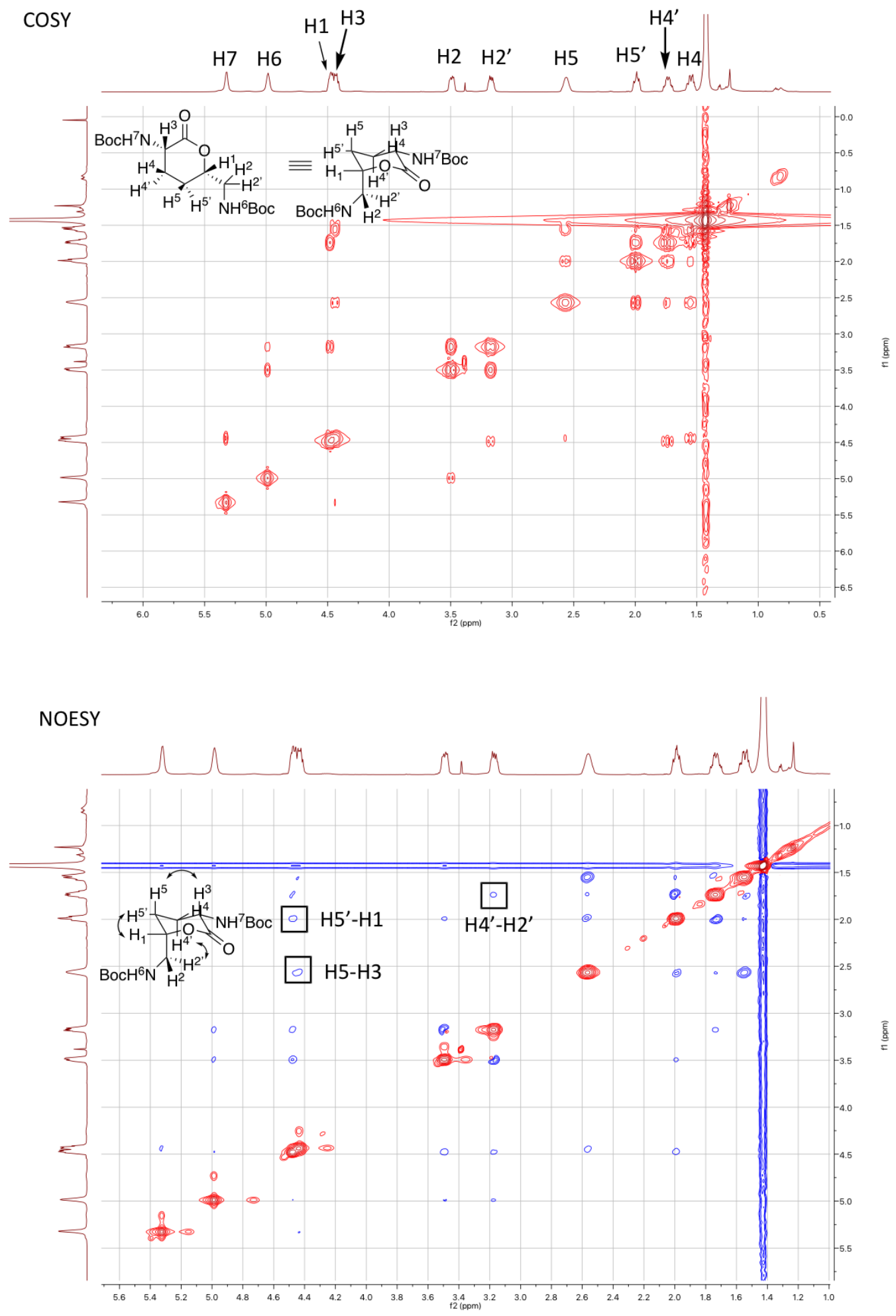

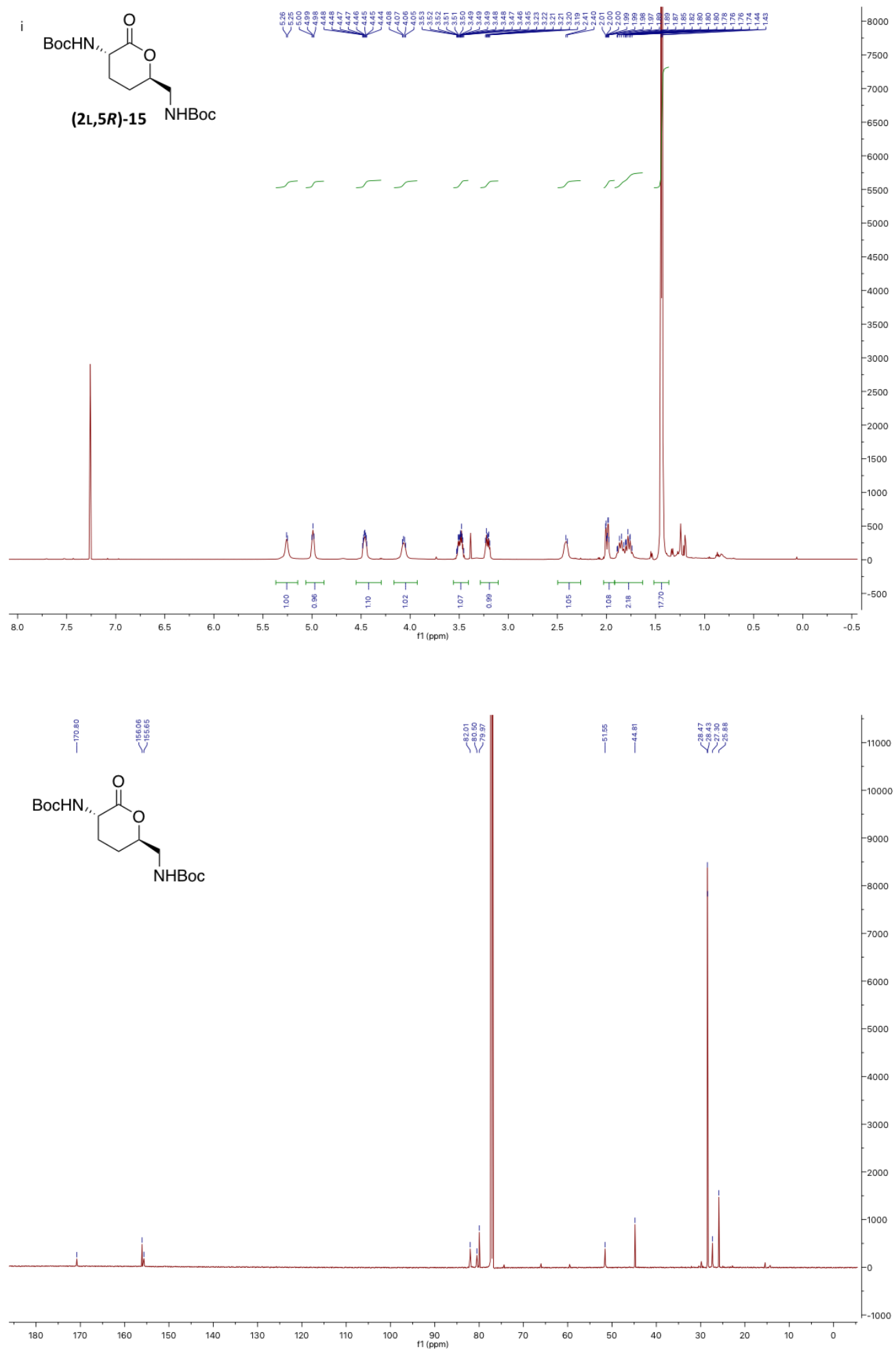
COSY
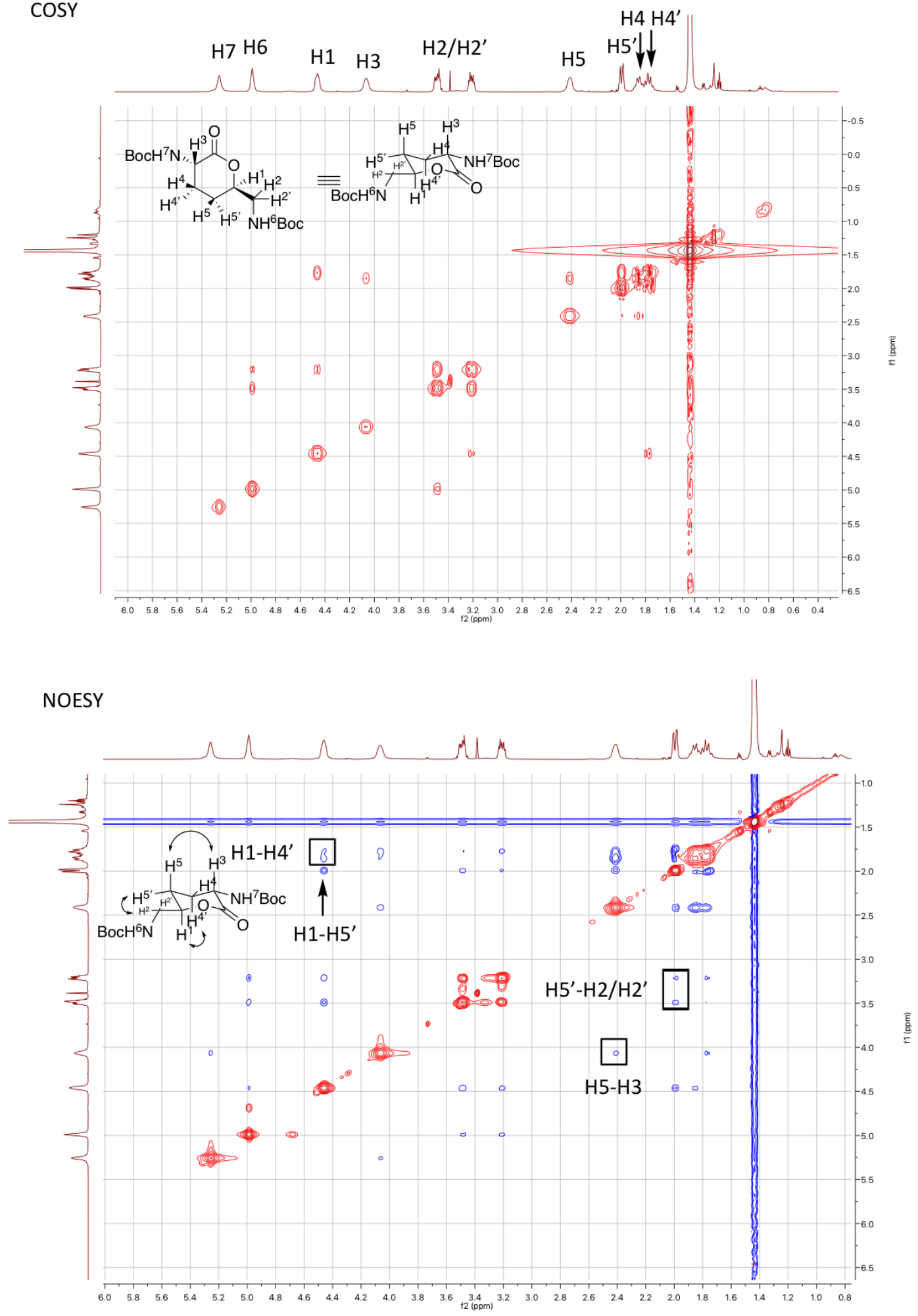

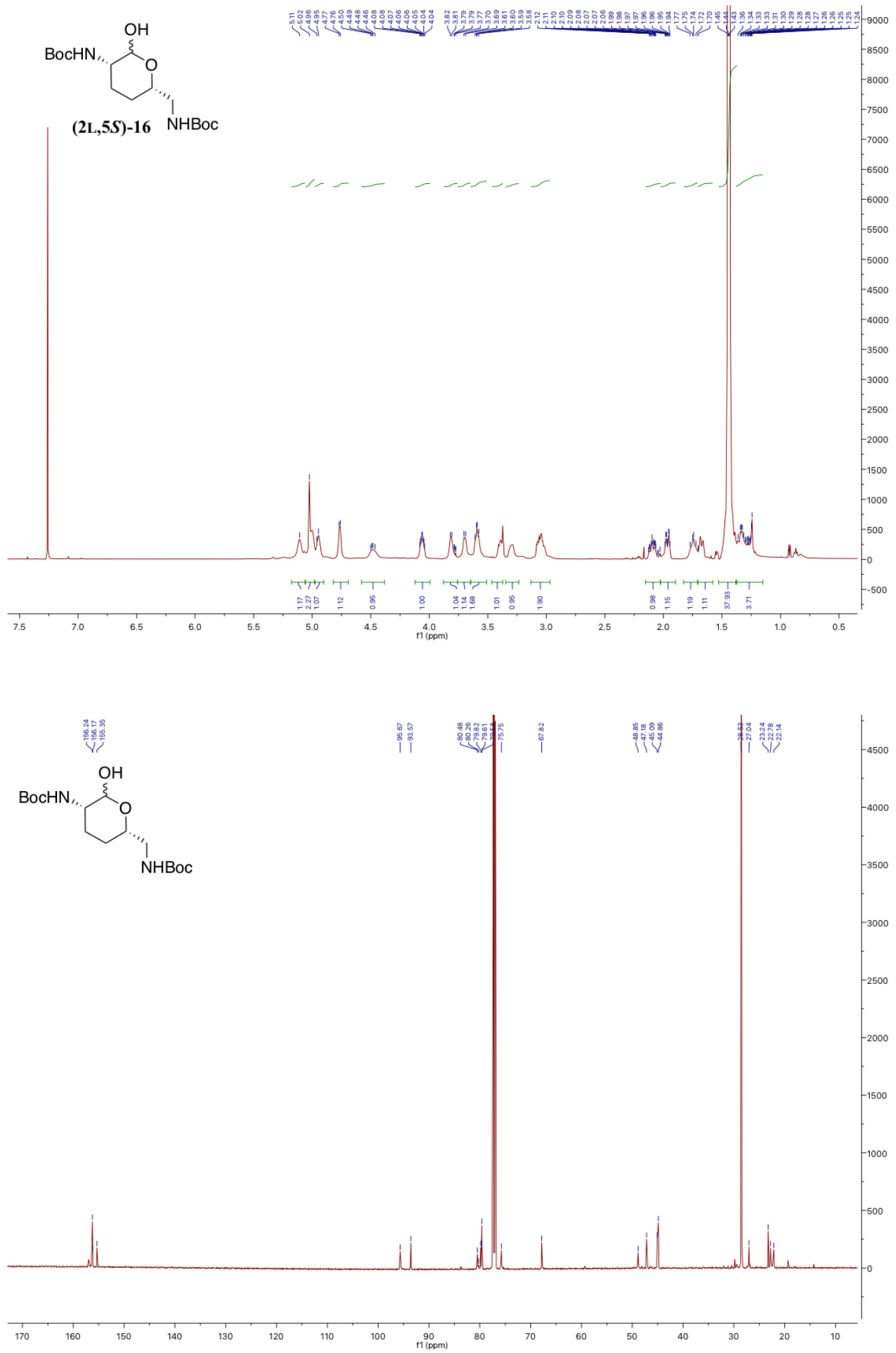

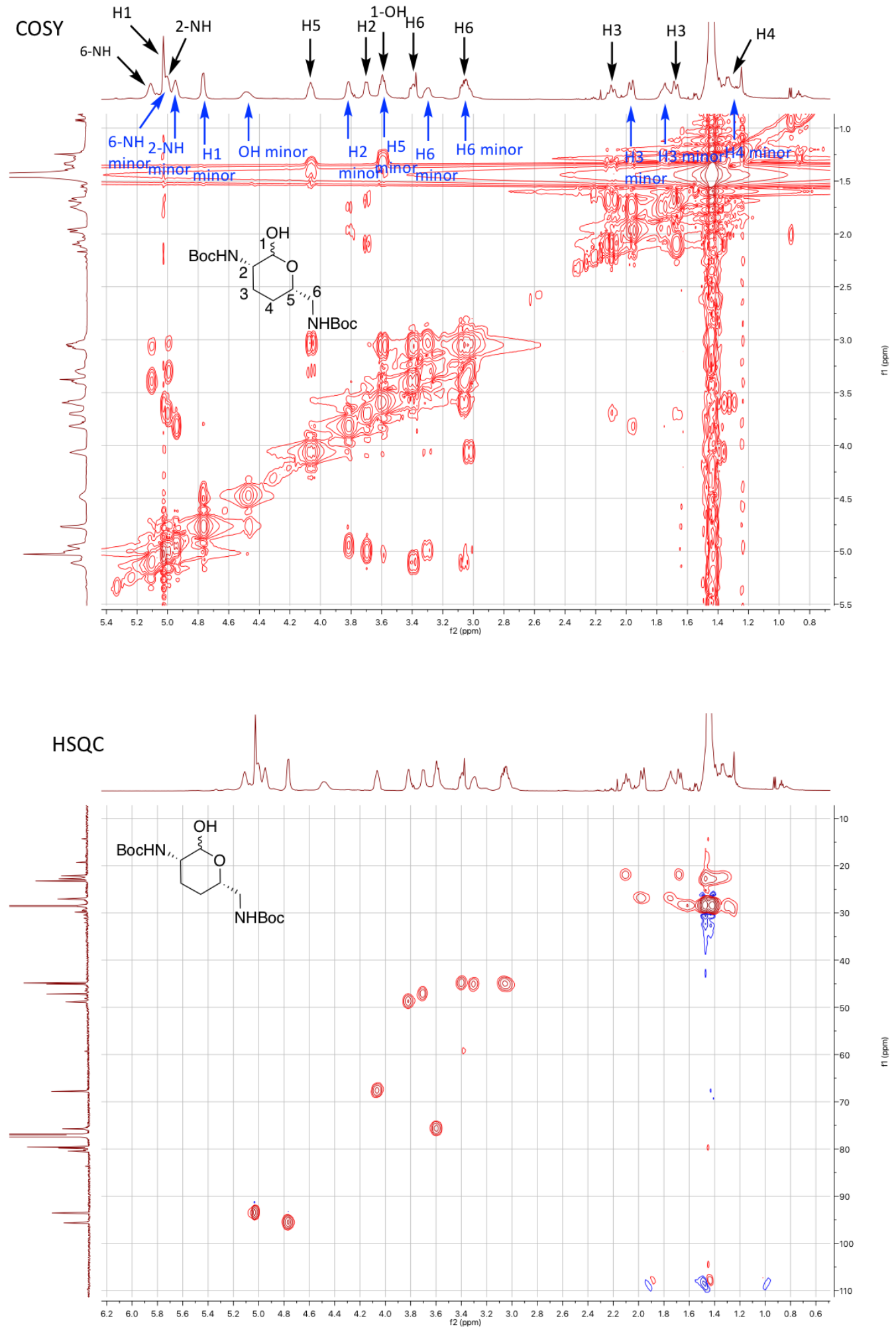

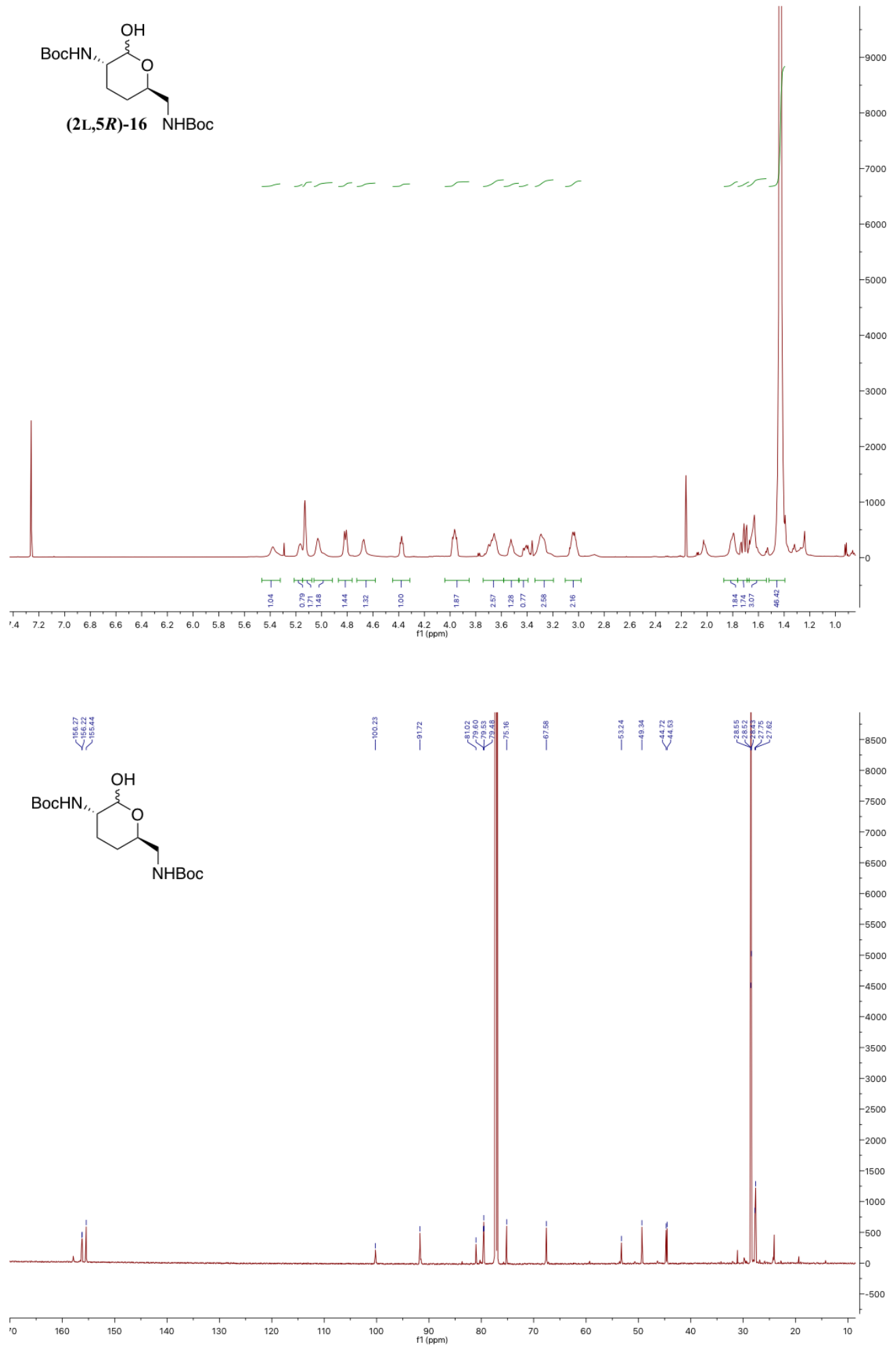

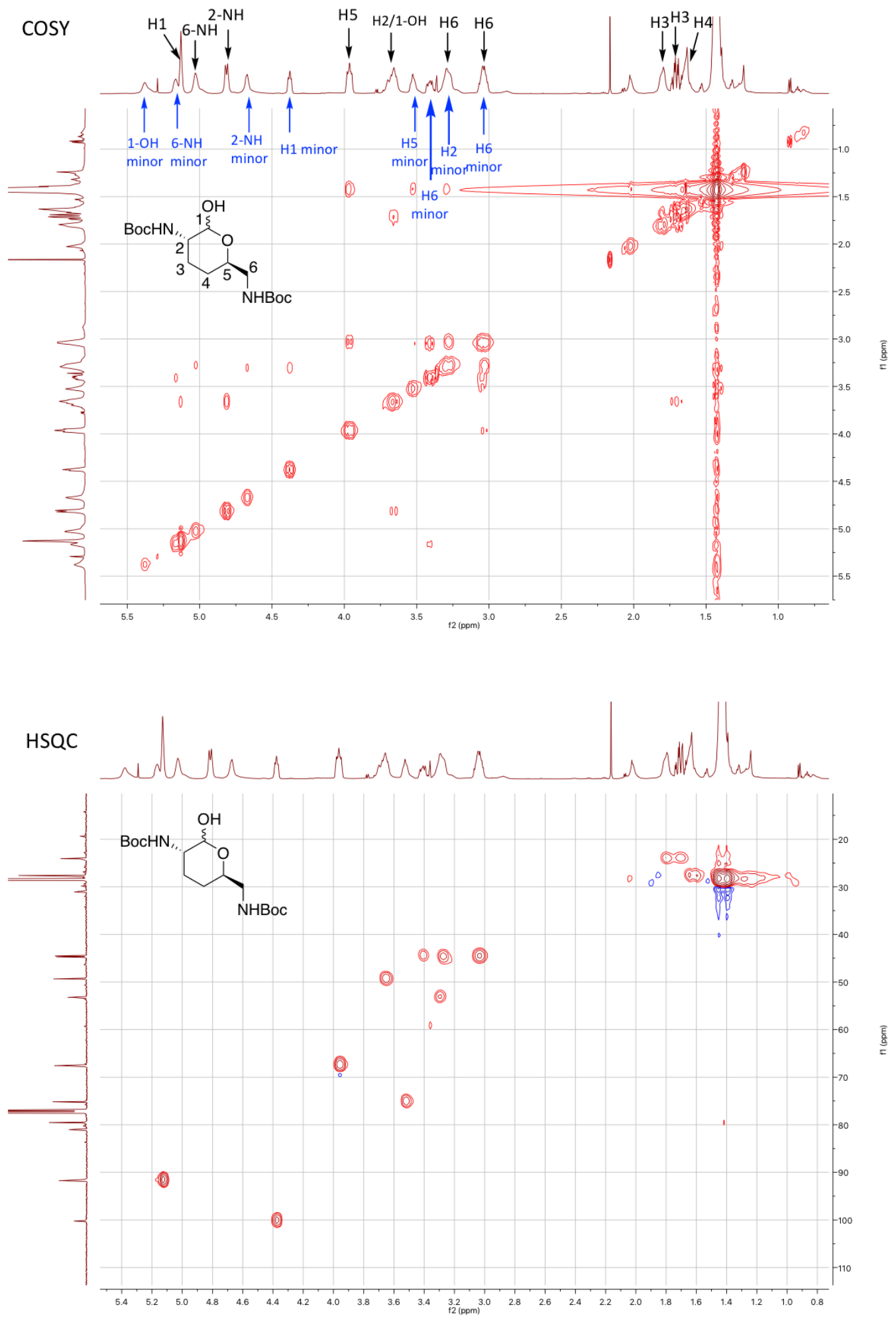


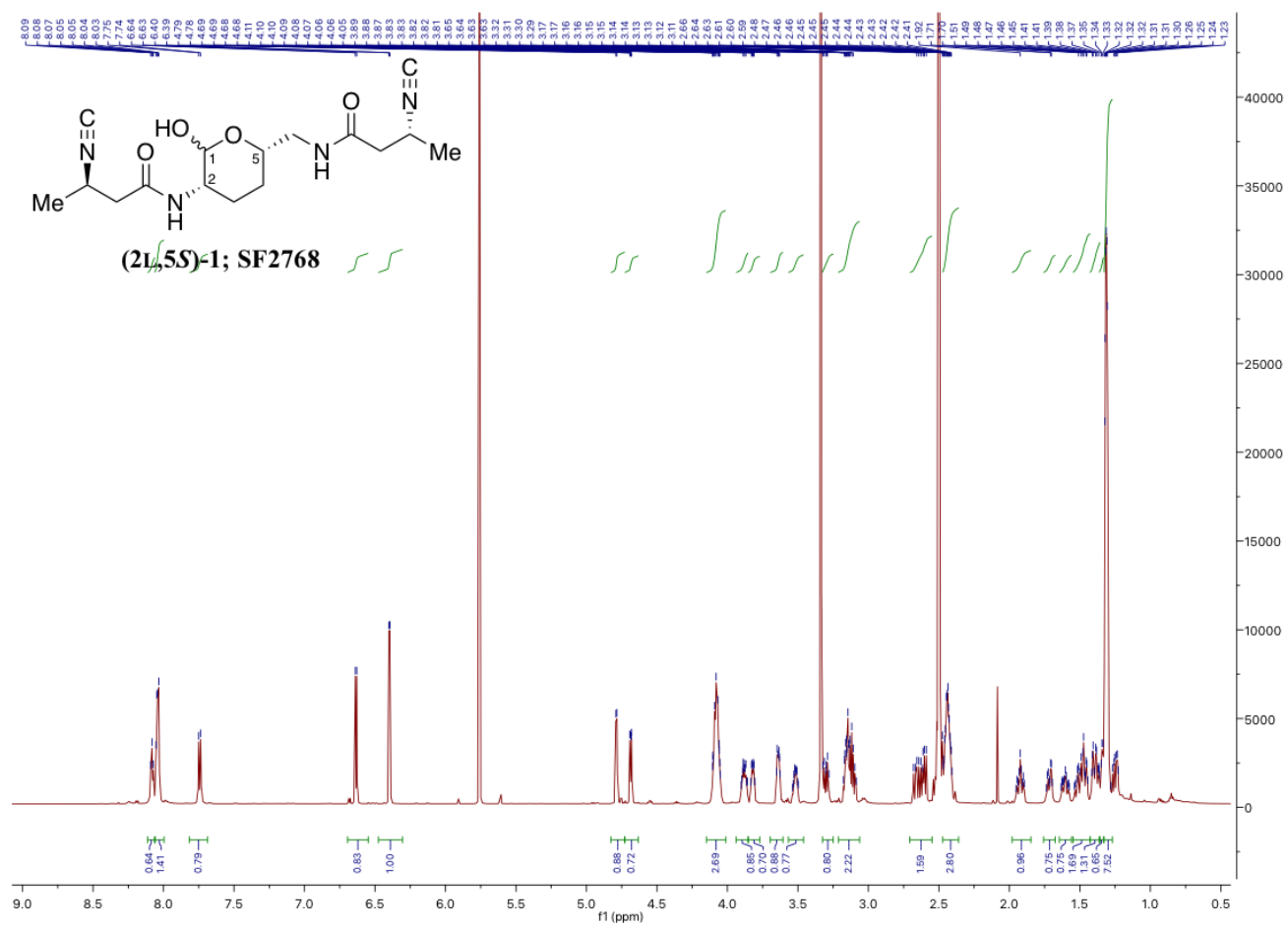

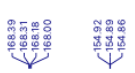<smiles>C=NC(=O)CCC1=CCC(NCC(=O)NC(C)CC(=O)N=C)=C(O)O1</smiles>

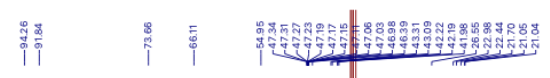
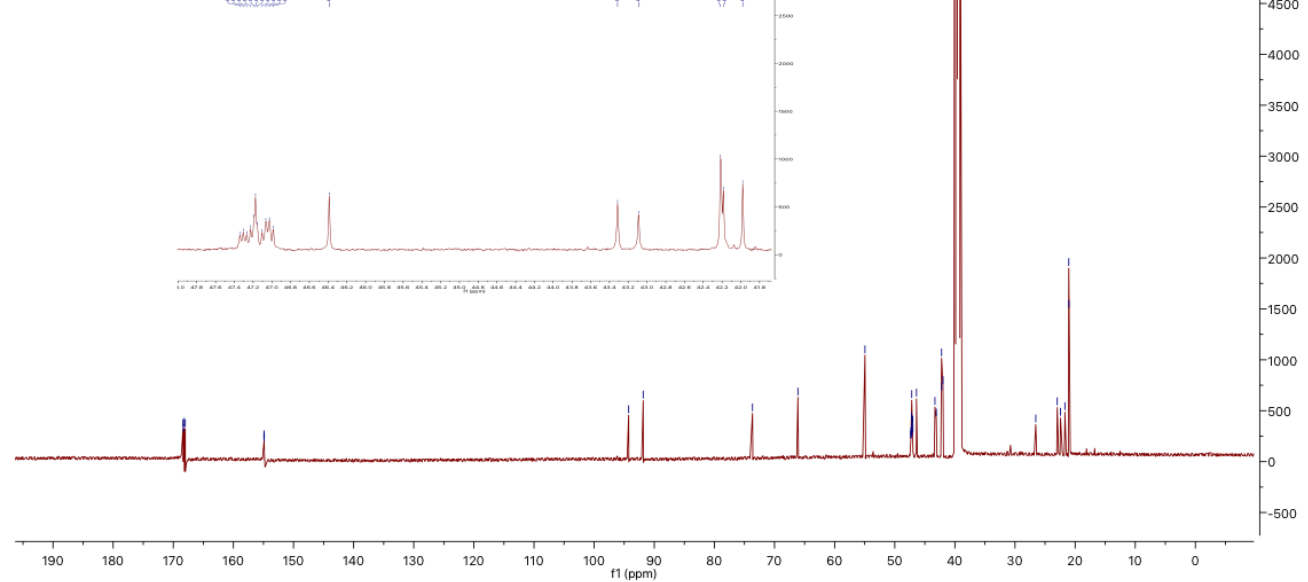

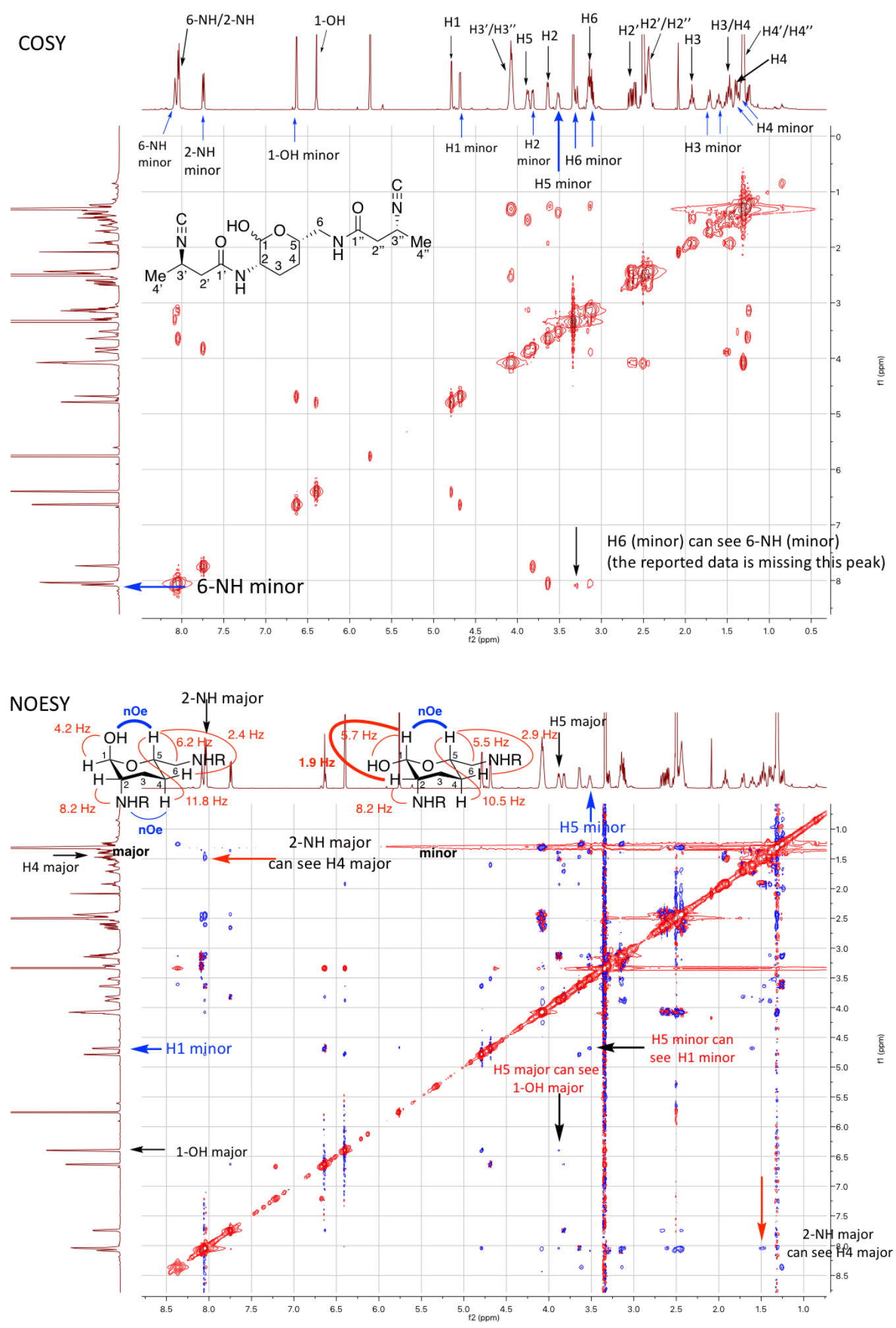

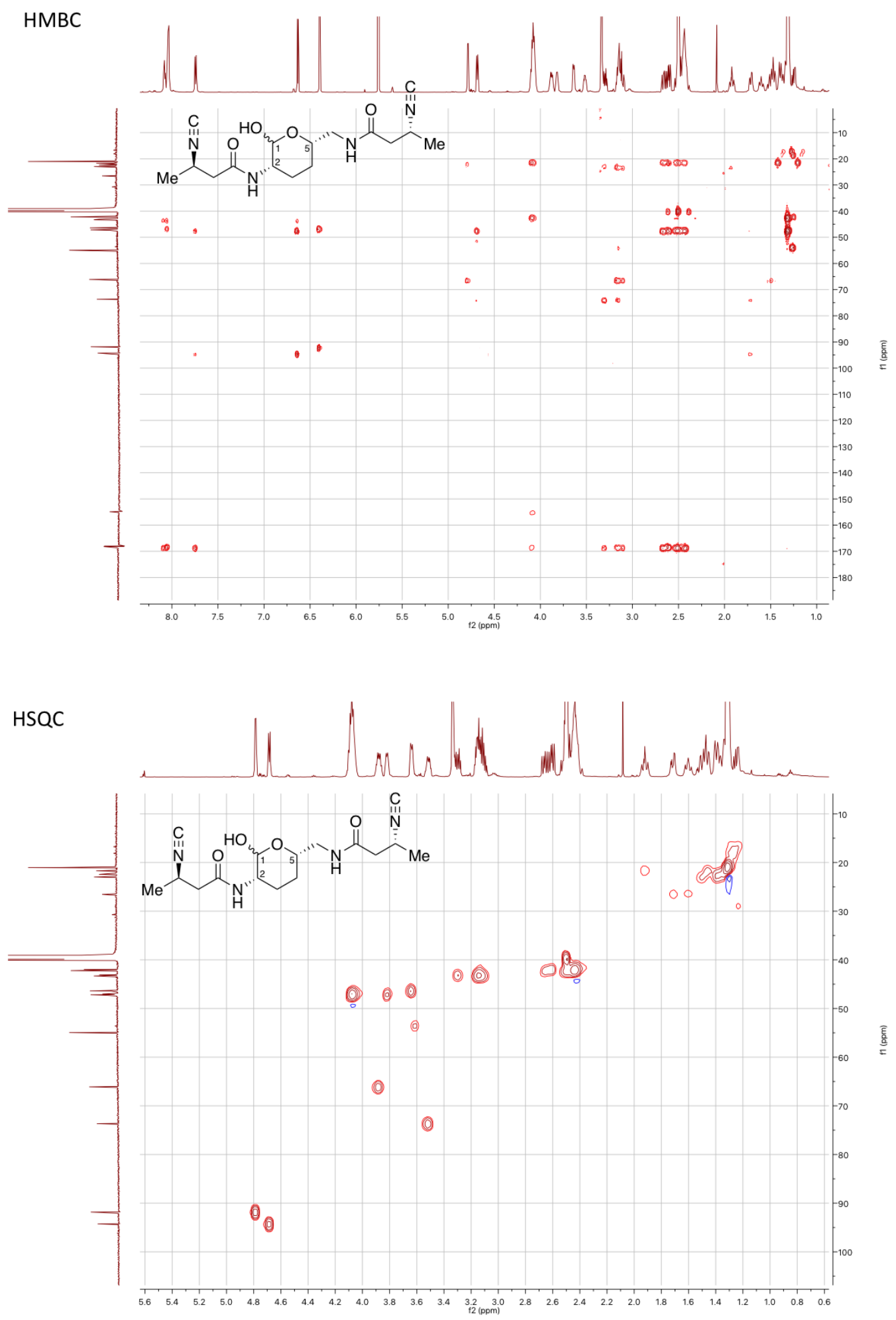
GTNDDRO PHOTON PAAMMETSOS

Pulse Sequence gresat

Solvent: Dao

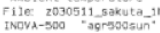

Pulse 90.0 degrees

Aco
M.

OASERVE H1. $499.9582839 \mathrm{NHZ}$

Tisize 65536
rotel tine 0 min, 56 sex
Spectrum of authentic SF2768 in $\mathrm{D}_{2} \mathrm{O}$

was obtained from Professor Kenji Ueda
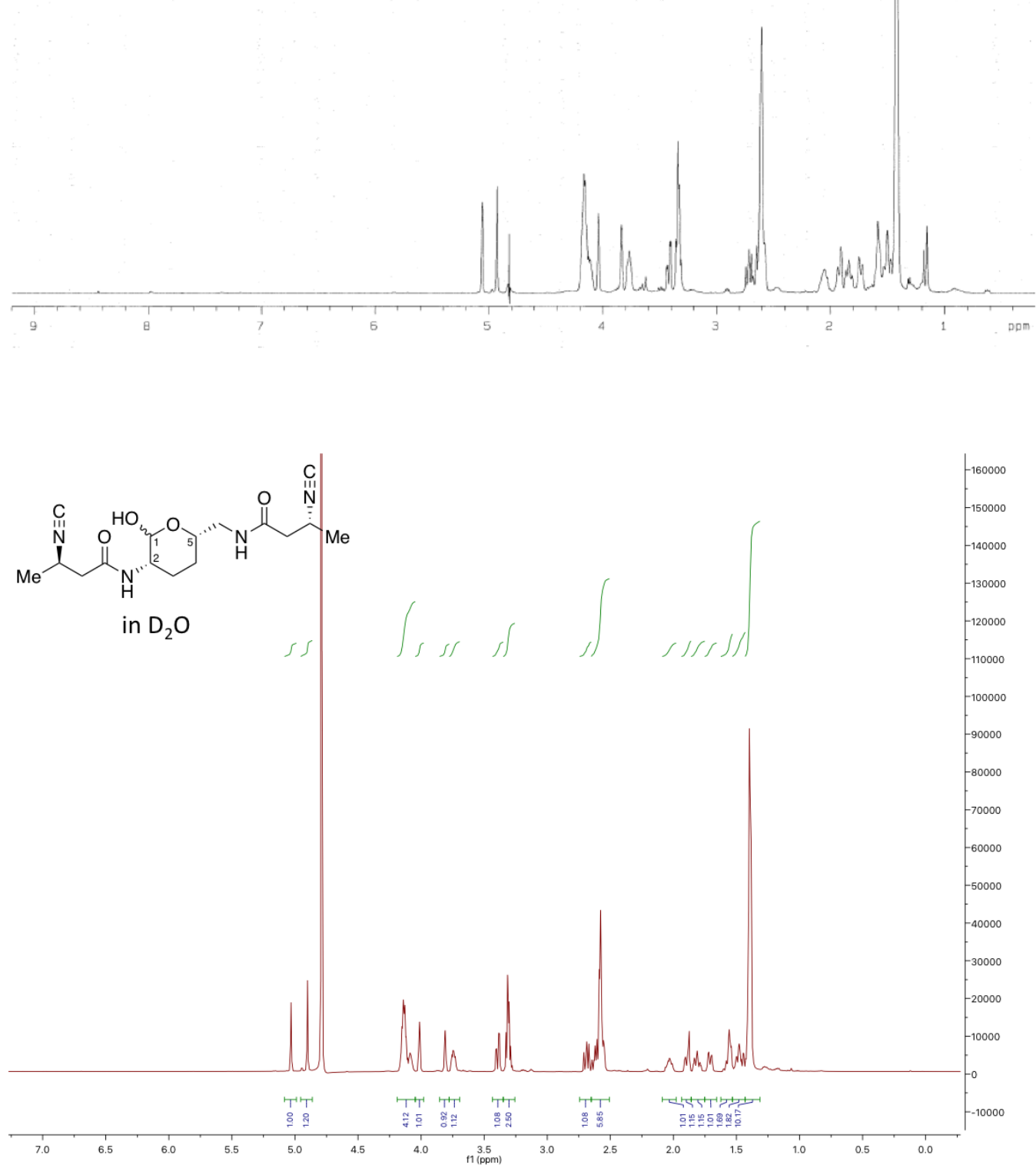

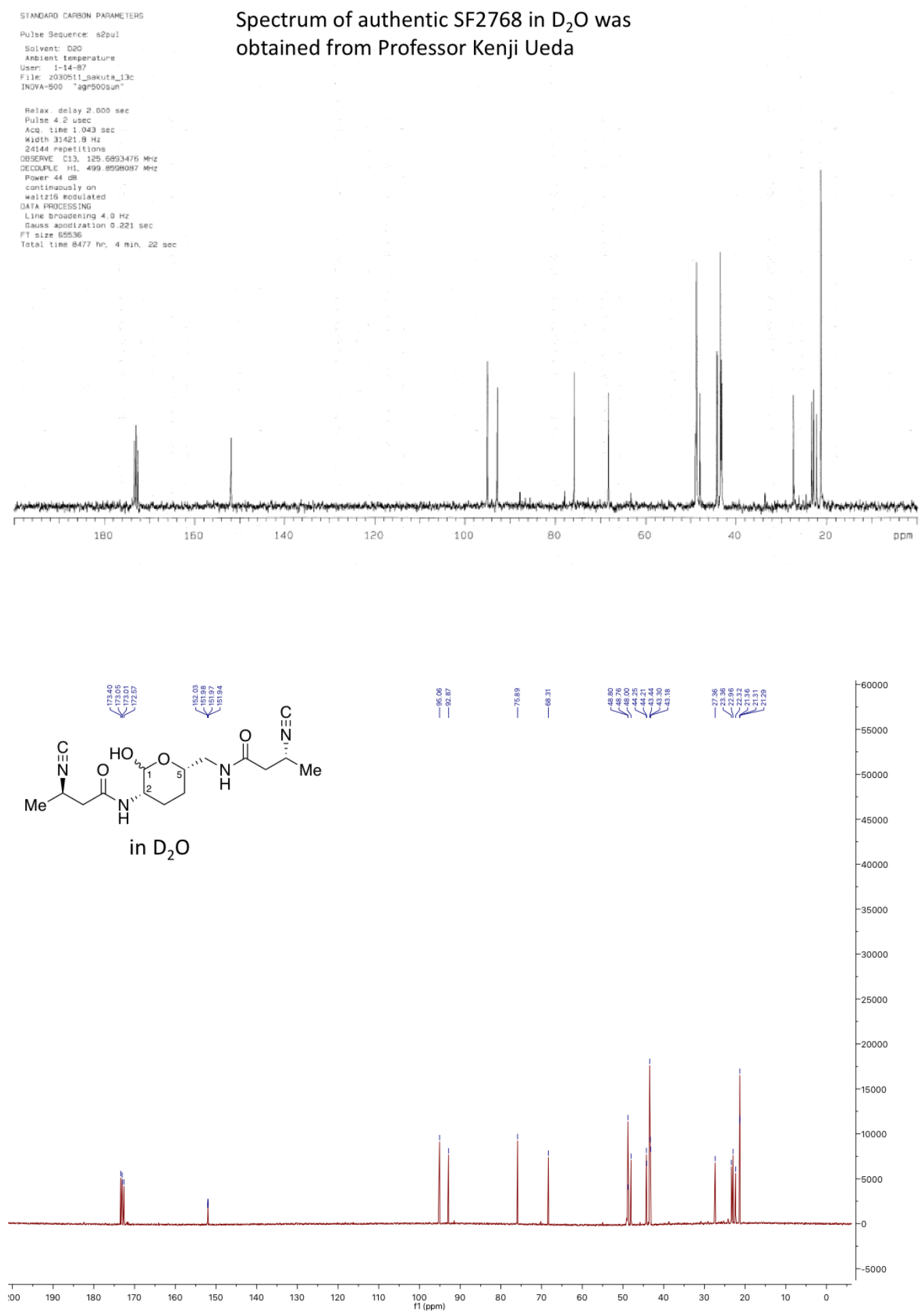

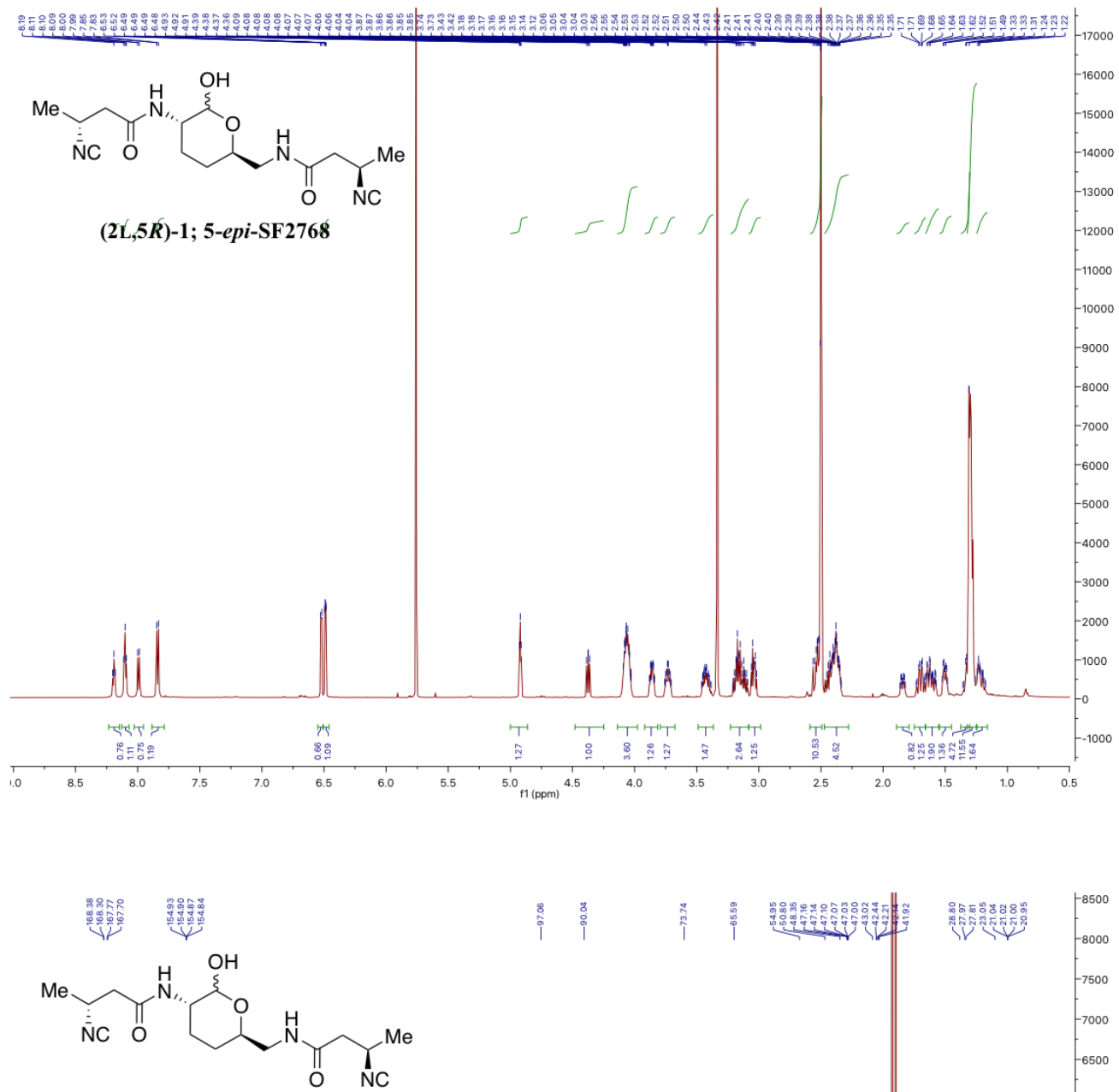

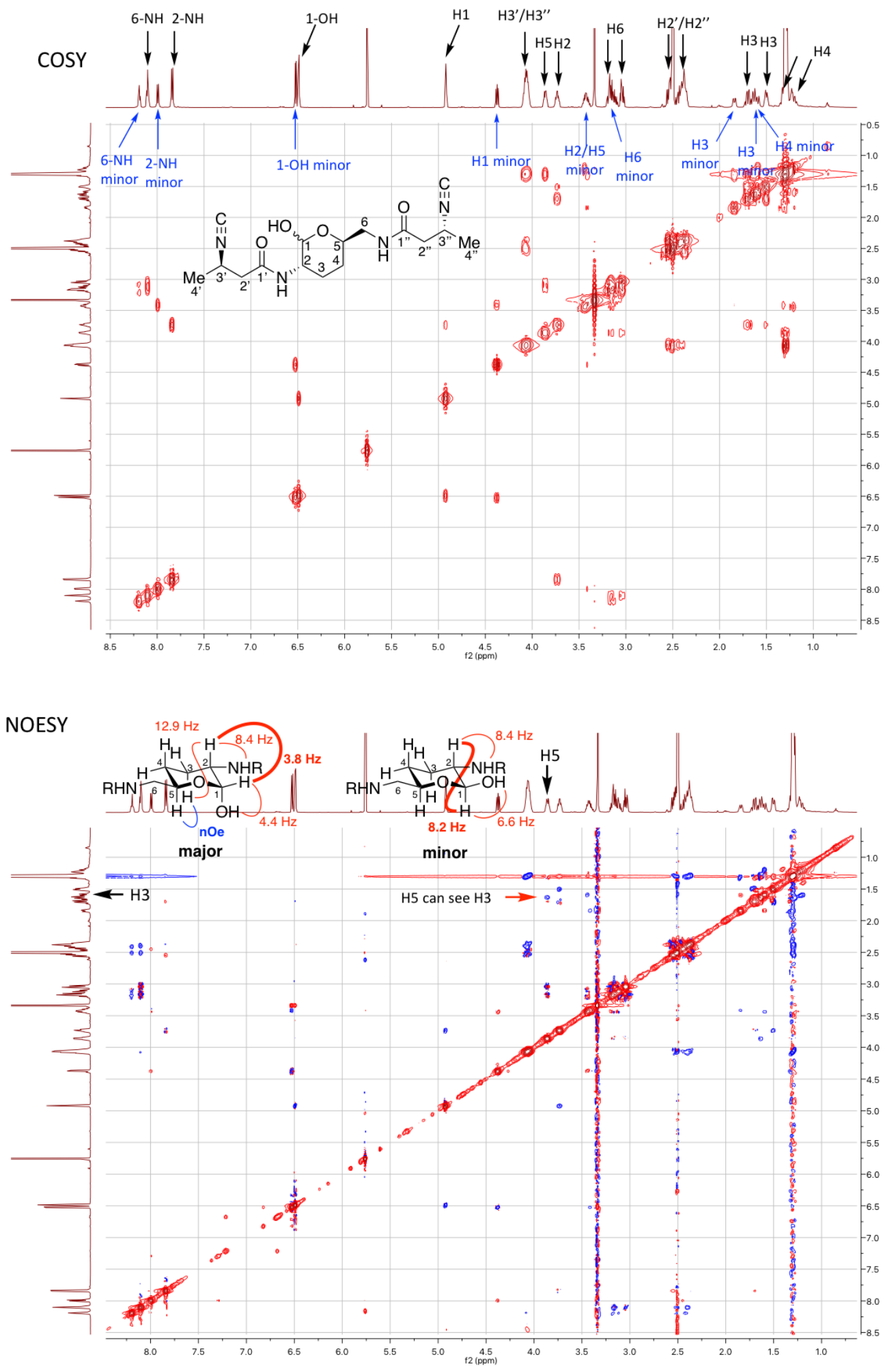

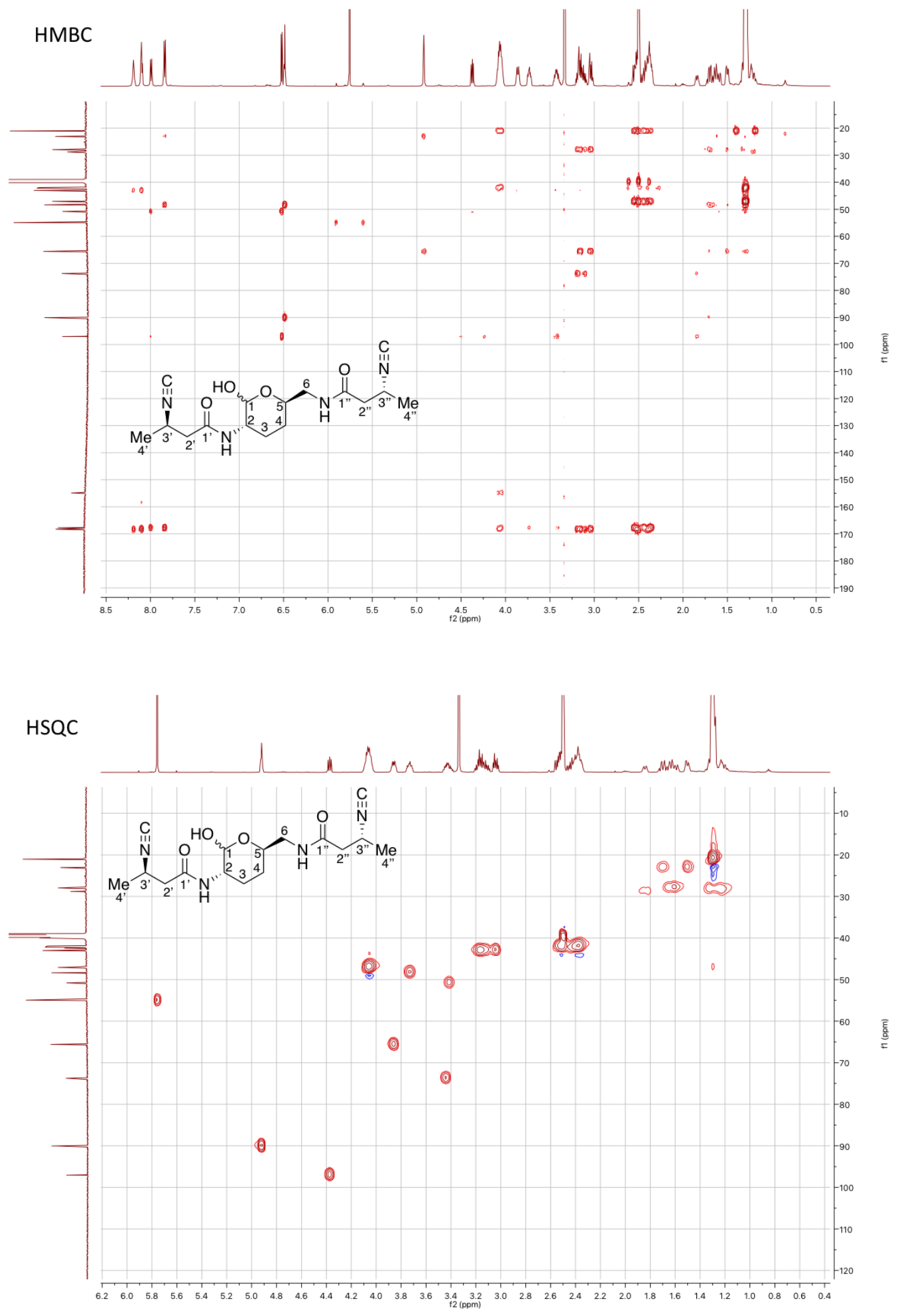

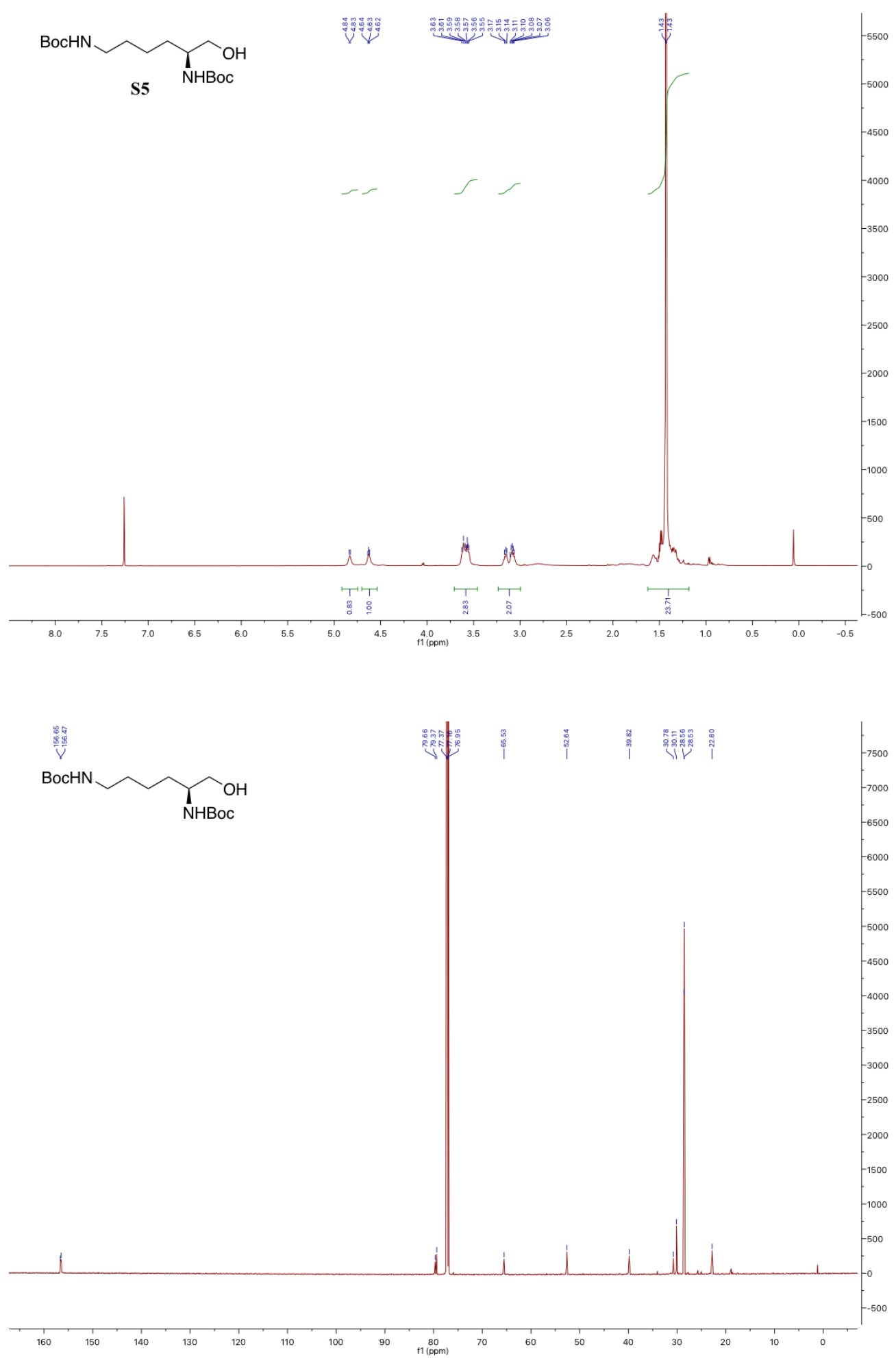


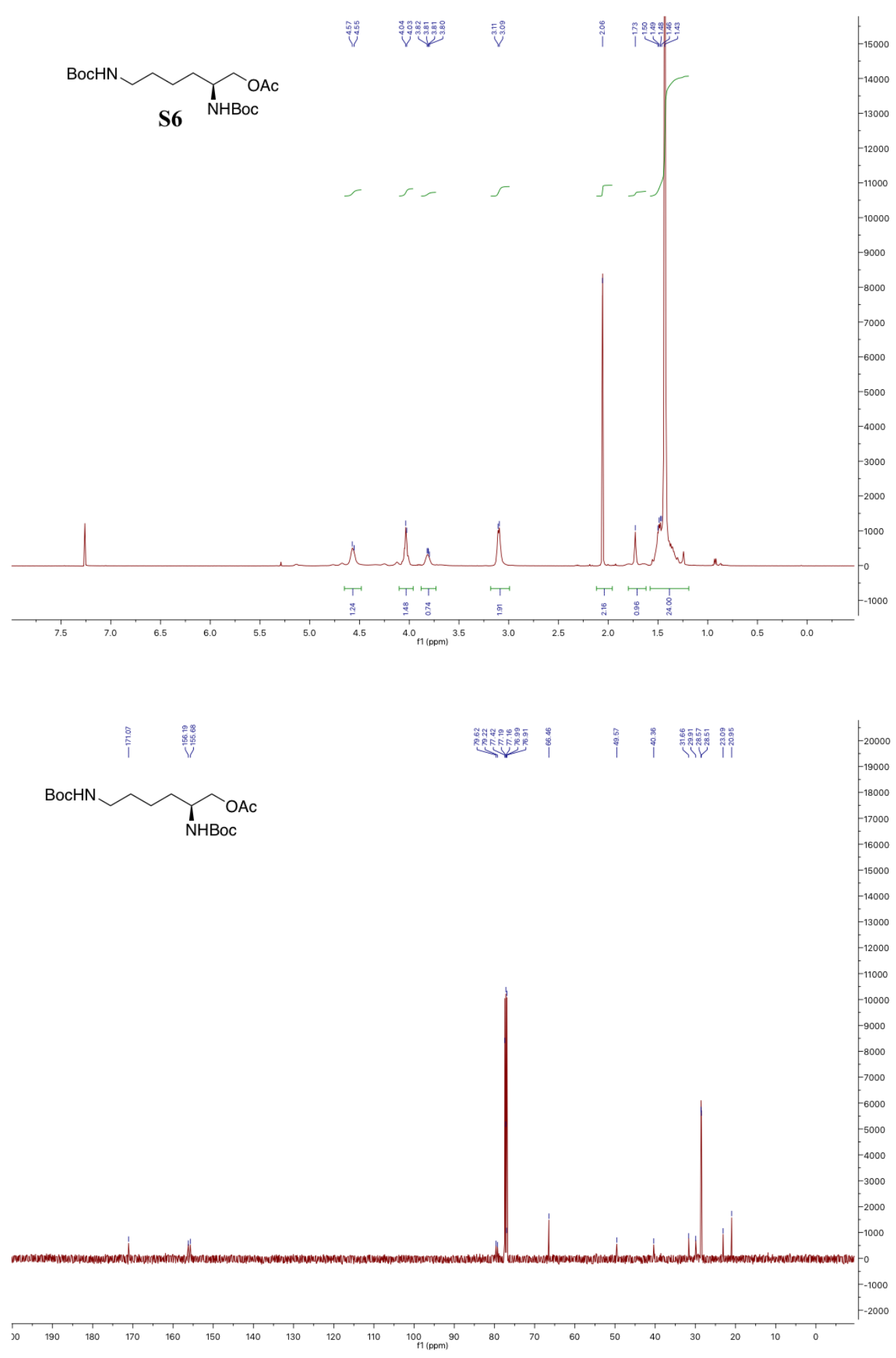



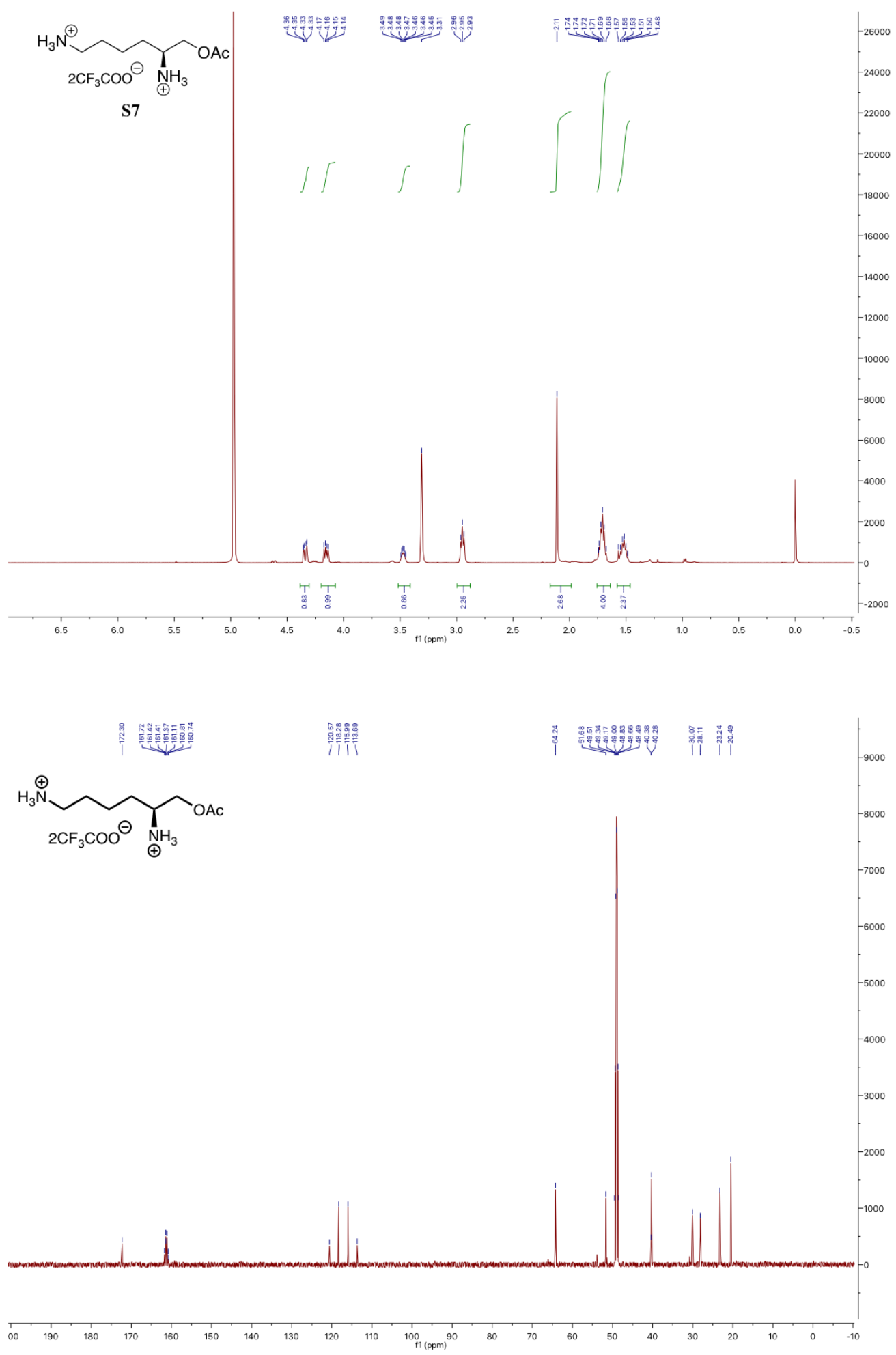

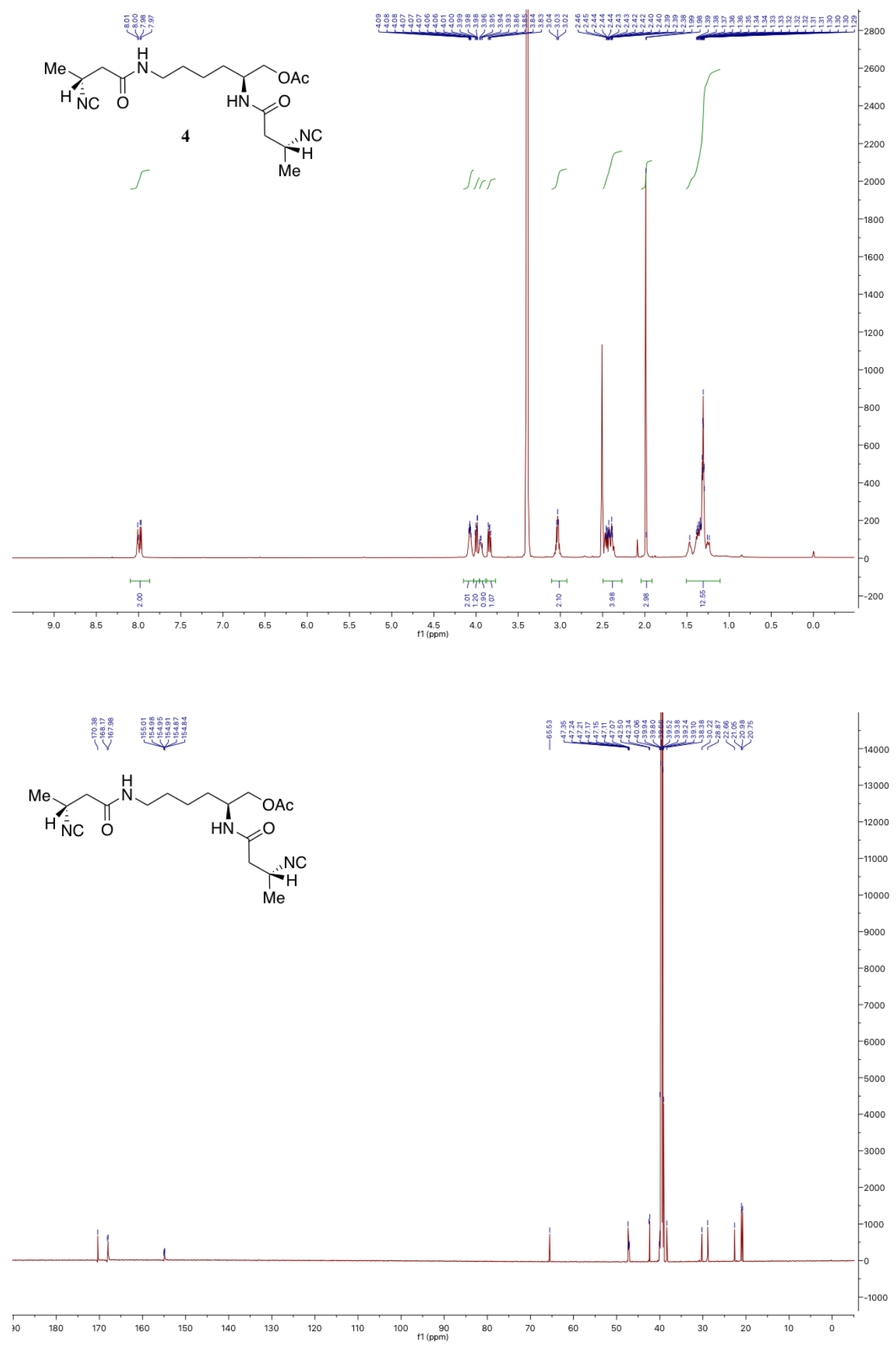

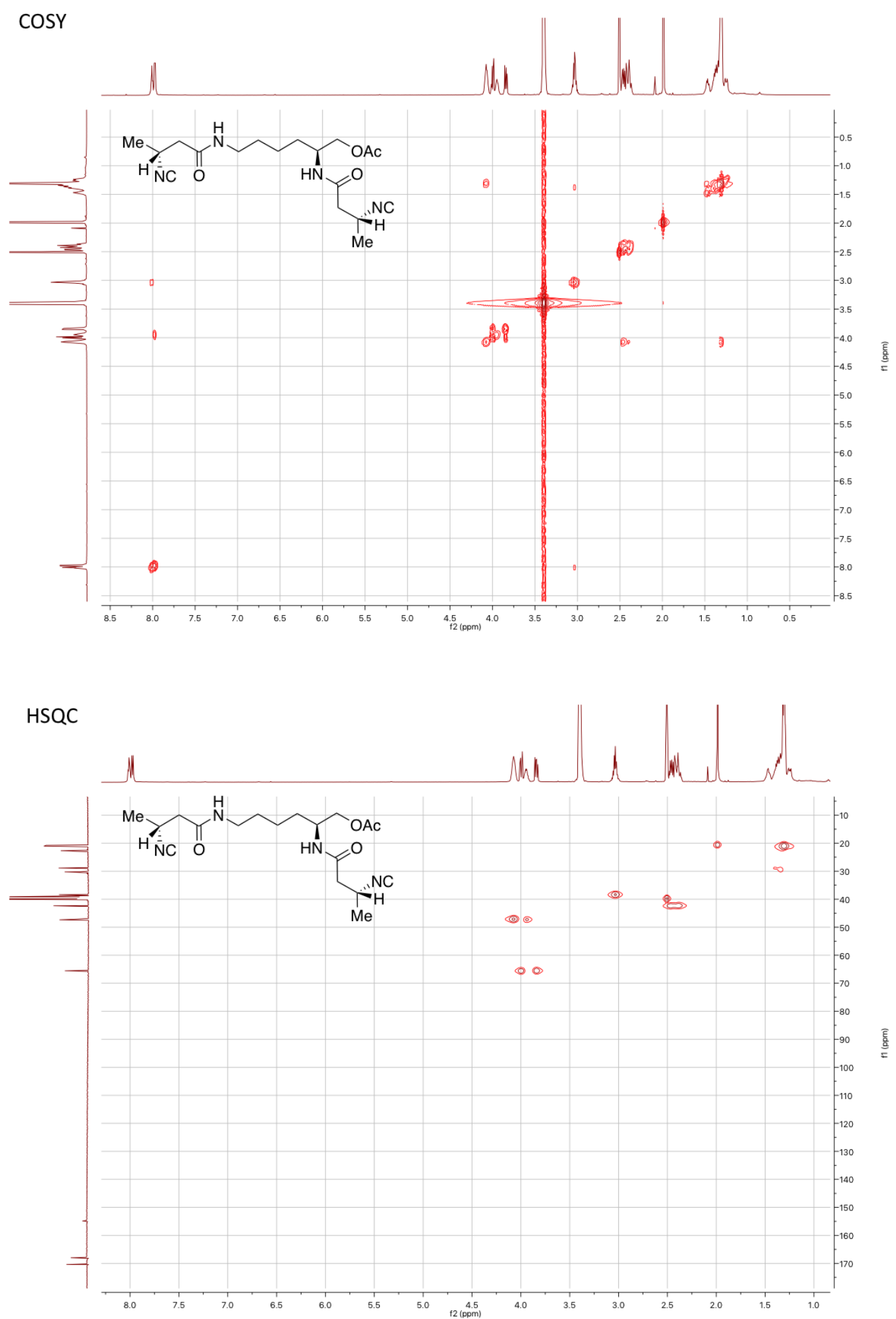

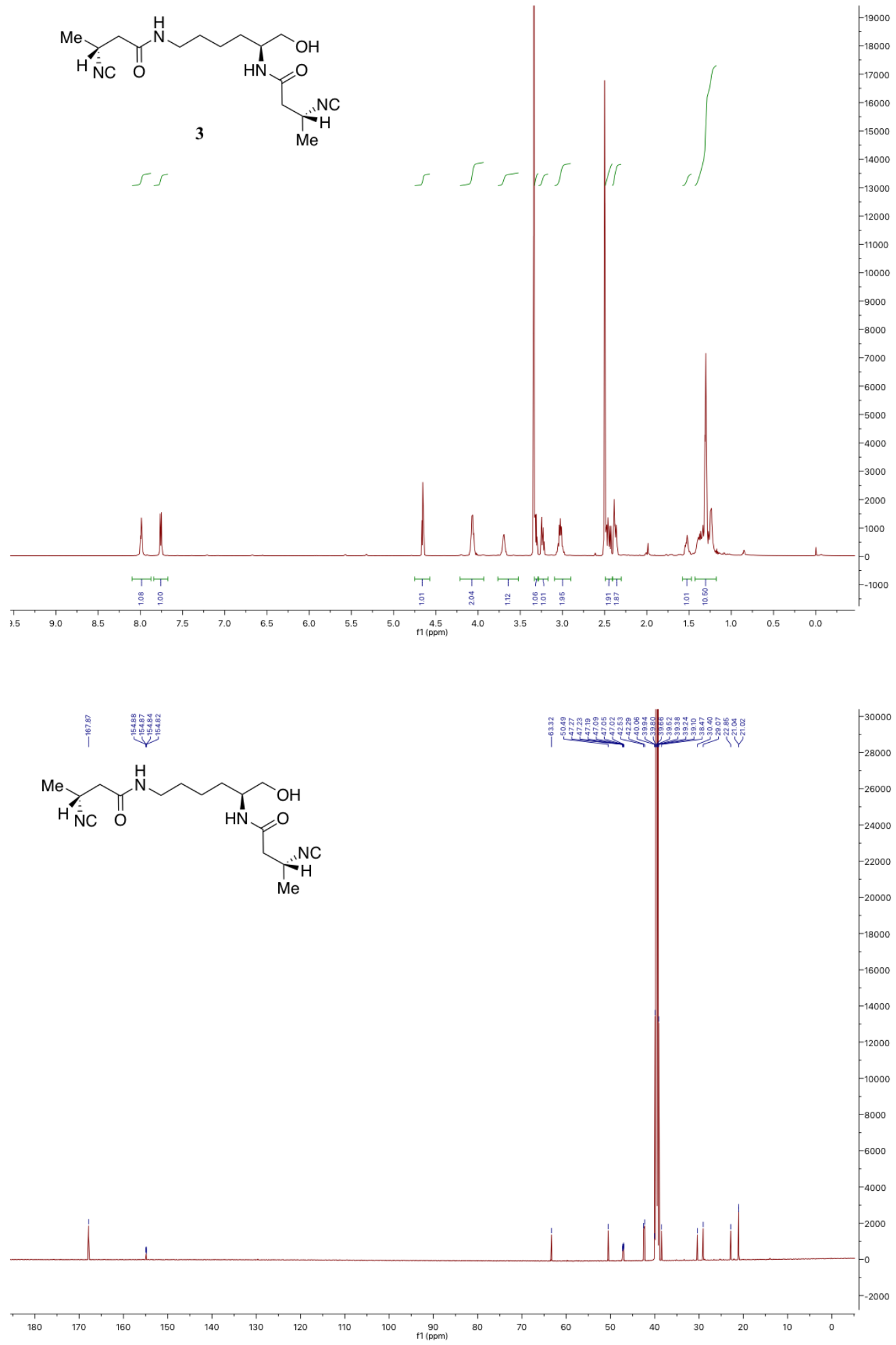

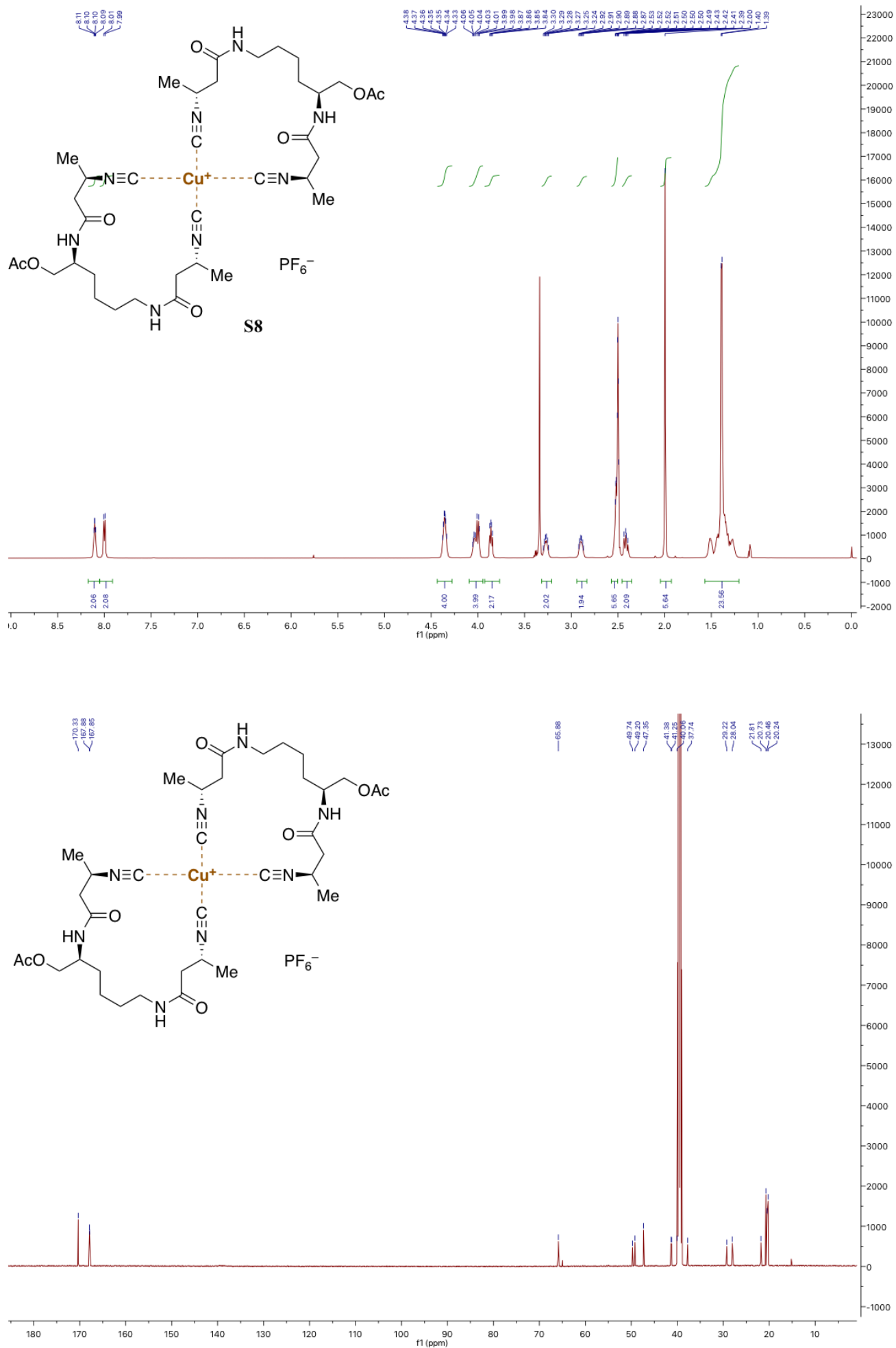

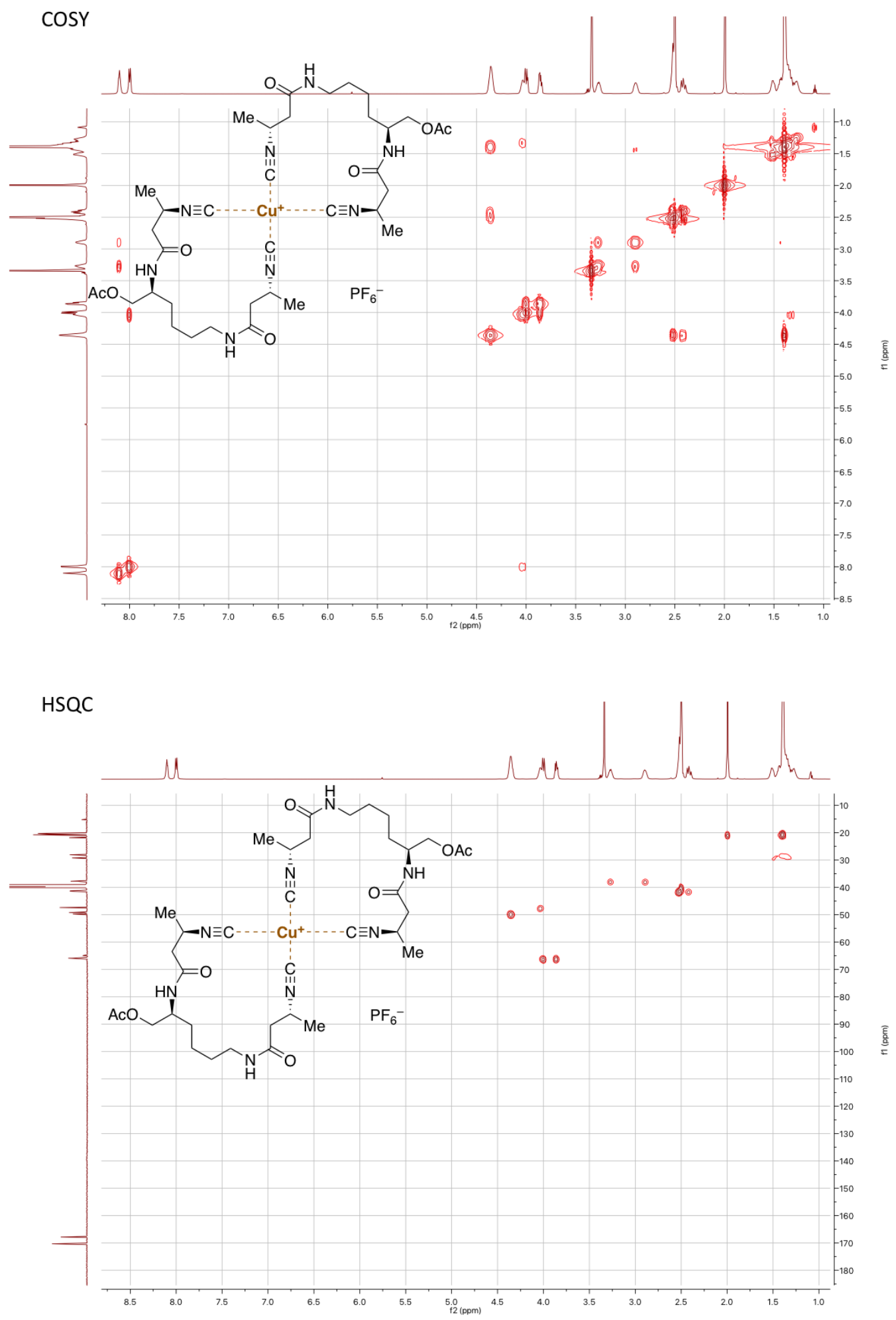


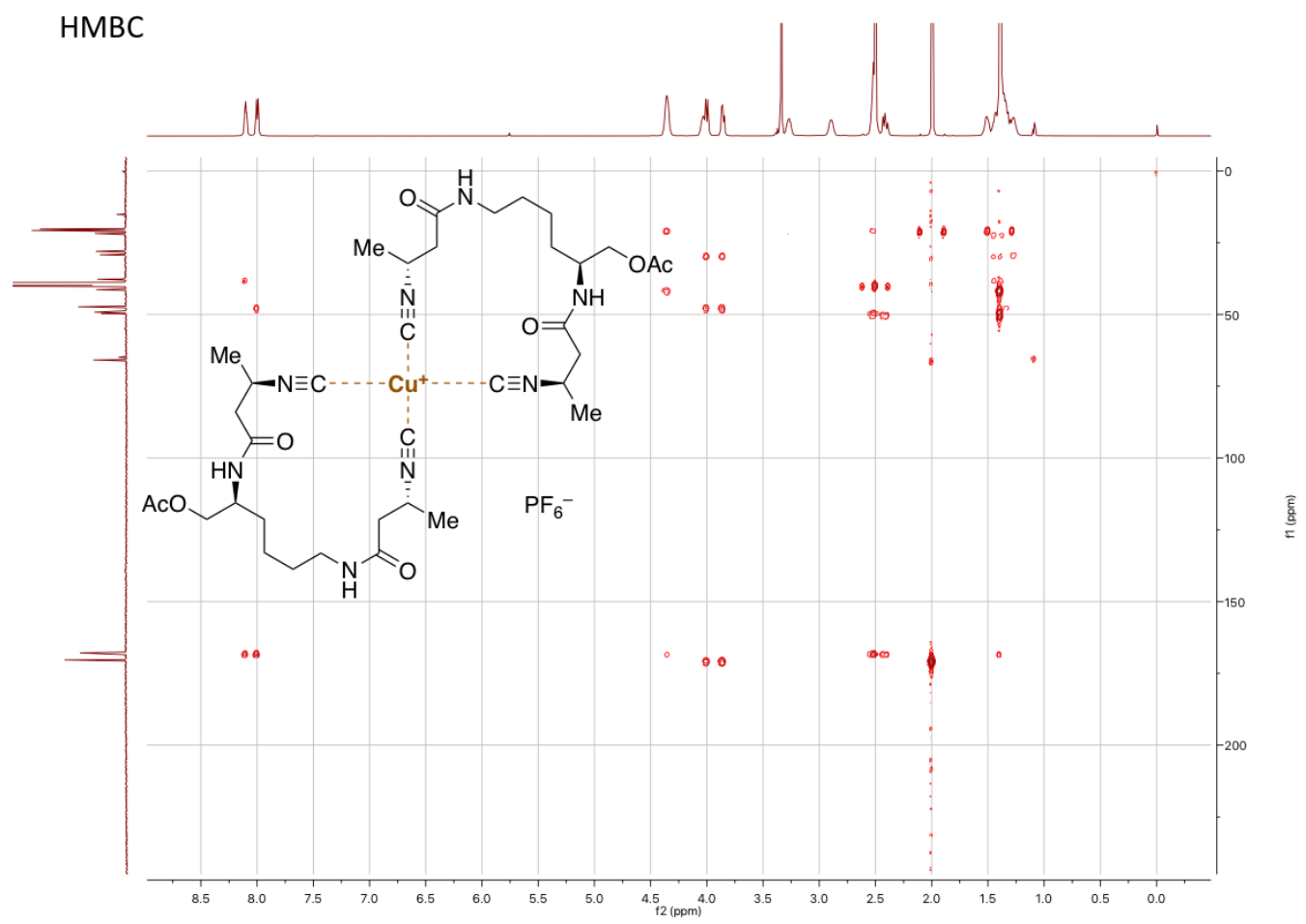

